\title{
Fingerprint Growth Prediction, Image Preprocessing and Multi-level Judgment Aggregation
}

\section{Dissertation}

\author{
zur Erlangung des \\ mathematisch-naturwissenschaftlichen Doktorgrades \\ "Doctor rerum naturalium" \\ der Georg-August-Universität Göttingen
}

vorgelegt von

Carsten Gottschlich

aus Bückeburg

Göttingen 2010 
Referent:

Koreferent:

Tag der mündlichen Prüfung:
Prof. Dr. Axel Munk

Prof. Dr. Preda Mihăilescu

26.04 .2010 


\section{Contents}

1 Introduction 1

1.1 Thesis Contribution ................... 1

1.1.1 Fingerprint Growth Analysis and Prediction . . . . . . . . 1

1.1.2 Fingerprint Image Preprocessing and Multi-level Judgment Aggregation .............. . . 3

I Fingerprint Growth $\quad 5$

2 Fingerprint Growth Analysis and Prediction $\quad 7$

2.1 Introduction . . . . . . . . . . . . . . . . 7

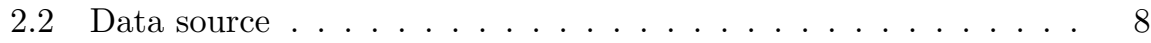

2.3 Growth Analysis . . . . . . . . . . . . . . . . . 8

2.4 Results of Validation Tests . . . . . . . . . . . . . . . 9

2.4.1 Test 1: Minutiae Distances after Alignment . . . . . . . . 9

2.4.2 Software for Minutiae Extraction and Matching . . . . . . 11

2.4.3 Test 2: Verification Tests . . . . . . . . . . . . 12

2.4.4 Test 3: Identification Test at the BKA . . . . . . . . . . 14

2.5 Anecdotes . . . . . . . . . . . . . . . . . . . . . . 15

2.6 Discussion . . . . . . . . . . . . . . . . . 16

II Fingerprint Image Preprocessing $\quad 17$

$\begin{array}{llr}3 & \text { Introduction } & 19\end{array}$

4 Orientation Field Estimation $\quad 21$

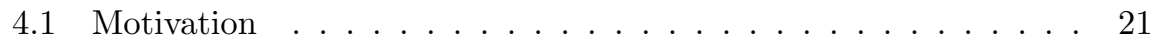

4.2 Review of Existing Methods . . . . . . . . . . . . . . . . 21

4.3 Line Sensor Based OF Estimation . . . . . . . . . . . . . . . 24

4.3.1 Sketch of the Method . . . . . . . . . . . . . 24

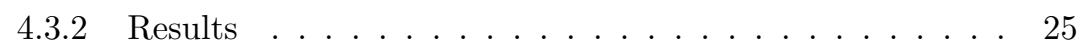

4.4 Detailed Description of the Line Sensor . . . . . . . . . . . . . 26

4.4.1 Preprocessing . . . . . . . . . . . . . . 26

4.4.2 Orientation Field Estimation . . . . . . . . . . . . . 29

4.4.3 Segmentation and Image Quality . . . . . . . . . . . . . . . . . 34

4.4.4 Ridge Frequency Estimation . . . . . . . . . . . . . 36 
4.5 Experimental Results . . . . . . . . . . . . . . . . . . 36

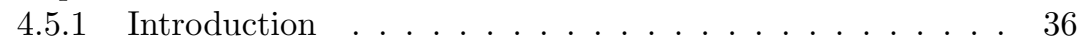

4.5.2 Scar Simulation . . . . . . . . . . . . . . . . . 36

4.5.3 Simulation of Broken Ridges _ . . . . . . . . . . . 37

4.5.4 Real Life Noise . . . . . . . . . . . . . . . . . . . . 38

4.6 Discussion . . . . . . . . . . . . . . . . . . . 42

4.6.1 Application Areas and Computational Complexity . . . . 42

4.7 Fast Line Sensor . . . . . . . . . . . . . . . . . . . . 42

4.7 .1 Preprocessing . . . . . . . . . . . . . . . . . 44

4.7 .2 Line Sensing . . . . . . . . . . . . . . . . . . . . . . . 44

4.7 .3 Coherent Structure . . . . . . . . . . . . . . . . . 46

4.7.4 Orientation Field Estimation . . . . . . . . . . . . 46

4.7 .5 Parameters . . . . . . . . . . . . . . . 46

4.7 .6 Discussion . . . . . . . . . . . . . . . . . 48

5 Line Sensing Based on

Quadratic Bezier Splines

6 Ridge Frequency Estimation $\quad 55$

6.1 Related Work . . . . . . . . . . . . . . . . . . . . . . 57

$6.1 .1 \quad$ X-Signature . . . . . . . . . . . . . . . . . . 57

6.1 .2 Higher Order Spectrum . . . . . . . . . . . . . 57

6.1.3 Geometric and Spectral Approach _. . . . . . . . . 57

6.1.4 Short Time Fourier Transform . . . . . . . . . . . . . 57

6.1.5 Sinusoidal Shaped Surface . . . . . . . . . . . . . . . 57

6.1.6 Further Methods . . . . . . . . . . . . . . . . . . 57

6.2 Curved Regions . . . . . . . . . . . . . . . . . 58

7 Image Enhancement $\quad 65$

7.1 Related Work . . . . . . . . . . . . . . . . . . . . . 66

7.1.1 Pyramid Methods . . . . . . . . . . . . . . . . 66

7.1.2 Short Time Fourier Transform . . . . . . . . . . . 66

7.1.3 Discrete Cosine Transform . . . . . . . . . . . . . . . . 67

7.1.4 Wavelet Transform . . . . . . . . . . . . . . . 67

7.1 .5 Gabor Filter . . . . . . . . . . . . . . . 67

7.1.6 Anisotropic Filtering . . . . . . . . . . . . . . . . 67

7.1 .7 Further Methods . . . . . . . . . . . . . . . . 67

7.2 Gabor Filter . . . . . . . . . . . . . . . . . . 67

$7.2 .1 \quad$ Filter Parameters and Their Impact . . . . . . . . . 68

$7.2 .2 \quad$ Gabor Filter Modifications . . . . . . . . . . . . . . 70

7.3 Curved Gabor Filter . . . . . . . . . . . . . . . . . . . . 71

8 Multi-Level Judgment Aggregation $\quad 81$

8.1 Multi-Level Judgment Aggregation . . . . . . . . . . . . . . . 83

8.2 Test Setup . . . . . . . . . . . . . . . . . . . . . . . 84

8.3 Image Enhancement by Applying the Curved Gabor Filter . . . . 86

8.3.1 Results and Discussion . . . . . . . . . . . . . . . . 87

8.4 Score Fusion vs. Minutiae Fusion . . . . . . . . . . . . . . . 89

8.5 Image Quality Based Score Normalization . . . . . . . . . . . 91 
8.5.1 Test Details . . . . . . . . . . . . . . . . . . . . . . . 91

8.5.2 Results and Discussion . . . . . . . . . . . . . . . . 92

8.6 The Doddington Zoo Effect . . . . . . . . . . . . . . . 93

8.6 .1 Discussion . . . . . . . . . . . . . . . . . . . 94

8.7 Cross Matching . . . . . . . . . . . . . . . . . . . 95

8.8 Score Revaluation . . . . . . . . . . . . . . . . . . . 97

8.8 .1 Features . . . . . . . . . . . . . . . . 98

8.8.2 Case Study: FVC 2002 Database 2 . . . . . . . . . . . 99

8.8.3 Generalization of Revaluation Criteria . . . . . . . . . 104

8.8.4 Discussion . . . . . . . . . . . . . . . . . . . 105

8.9 Conclusion and Future Work _ . . . . . . . . . . . 107

$\begin{array}{ll}\text { Bibliography } & 112\end{array}$

$\begin{array}{ll}\text { Curriculum Vitae } & 124\end{array}$ 



\section{Acknowledgments}

First and foremost I would like to express my sincere gratitude to my advisor and mentor Axel Munk for personal and scientific supervision during the becoming of this thesis and his outright support in every regard. I am grateful for the opportunity to work on exciting and challenging topics from coinciding areas of pattern recognition, image processing and biometrics. I would like to thank my co-advisor Preda Mihăilescu for his inspiring ideas and suggestions, and his visionary, always optimistic thinking. Especially, I would like to thank him for our two weeks intensive course on contextual filtering. I appreciate their recommendations and their openness for discussions and questions. I would like to thank Thomas Hotz for the very pleasant joint work on the fingerprint growth, and especially for his statistical analysis of the data. Also, I am grateful for his considerate and thorough proof-reading of papers and posters. All people at the Institute for Mathematical Stochastics, in particular my work group colleagues Thomas Hotz, Krzysztof Mieloch and Stephan Huckemann, and my office-mates Sophie Bruns, Benjamin Tams and Jörn Dannemann (former office-mate), as well as Matthias Mielke, Johannes Schmidt-Hieber, Philipp Marnitz and Klaus Frick, I would like to thank for fruitful discussions and the enjoyable work atmosphere at the institute; the system administrators Krzysztof Mieloch and Julian Rüger for enabling the computations for this thesis, the people at the BKA in Wiesbaden, especially Robert Lorenz, Stefanie Bernhardt, Wolfgang Krodel and Michael Hantschel, for the interesting discussions, for the pleasant atmosphere during our visits and later communications and for providing invaluable data sets which made the fingerprint growth research possible in the first place.

I am grateful for the financial and scientific support by the DFG Research Training School 1023 "Identification in Mathematical Models: Synergy of Stochastic and Numerical Methods" The DFG RTS 1023 funded my participation at the "5th IAPR International Summer School for Advanced Studies on Biometrics for Secure Authentication" in Alghero, Italy, 2008, and the "6th International Symposium on Image and Signal Processing and Analysis" (ISPA), Salzburg, Austria, 2009 as well as the research visits to the BKA, Wiesbaden. The DFG Research Training School 1023 had in many ways an important positive influence: the 'Conference on Biometrical Feature Identification and Analysis' in Goettingen supported by the DFG RTS 1023 with invited speakers like John Daugman and Arun Ross was a motivating impulse at the beginning of my graduate studies. Later, during a numerics lecture held by Rainer Kress as a part of the DFG RTS 1023 curriculum, the idea of applying Bezier curves to fingerprint images was born which subsequently lead to the curved regions for 
ridge frequency estimation and the curved Gabor filter for image enhancement. In Alghero, I had the pleasure of listening to inspiring talks by Arun Ross, Josef Bigun and Josef Kittler, and in Salzburg, I was given the opportunity to present the line sensor based method for orientation field estimation and to listen to many stimulating talks including those by John Daugman and Vince Poor. The latter discussed "Collaborative Signal Processing in Wireless Sensor Networks" which became the inspiration for what subsequently resulted in multi-level judgment aggregation in fingerprint recognition. Pertaining to all mentioned events, I am thankful for the support of the DFG Research Training School 1023.

Carsten Gottschlich,

Göttingen, March 2010 


\begin{abstract}
Finger growth is studied in the first part of the thesis and a method for growth prediction is presented. The effectiveness of the method is validated in several tests. Fingerprint image preprocessing is discussed in the second part and novel methods for orientation field estimation, ridge frequency estimation and image enhancement are proposed: the line sensor method for orientation estimation provides more robustness to noise than state of the art methods. Curved regions are proposed for improving the ridge frequency estimation and curved Gabor filters for image enhancement. The notion of multi-level judgment aggregation is introduced as a design principle for combining different methods at all levels of fingerprint image processing. Lastly, score revaluation is proposed for incorporating information obtained during preprocessing into the score, and thus amending the quality of the similarity measure at the final stage. A sample application combines all proposed methods of the second part and demonstrates the validity of the approach by achieving massive verification performance improvements in comparison to state of the art software on all available databases of the fingerprint verification competitions (FVC).
\end{abstract}





\section{Chapter 1}

\section{Introduction}

Fingerprints have been used as a means of identification since ancient times [8]. In the late nineteenth century, first systematic studies of fingerprints and their properties were conducted by pioneers like Sir Francis Galton, Henry Faulds, Sir Edward Henry and Sir William Herschel [112]. Based on his empirical observations, Henry Faulds suggested in a letter to Nature in 1880 that "finger marks or impressions [..] may lead to the scientific identification of criminals." [13]. This statement implies two very important traits of fingerprints: permanence and individuality.

Sir Francis Galton, a cousin of Charles Darwin, was the first to define and name specific minutiae in his famous book "Finger Prints" published in 1892 [42]. The two most important types of minutiae are ridge endings and bifurcations, i.e. the division of a ridge into two ridges. Sir Edward Henry established a system for fingerprint classification called "the Henry System" in 1899 [56]. Similar fingerprint classification systems were also developed by Sir Francis Galton and Sir William Herschel [57]. These milestones were followed by the acceptance of fingerprint recognition as a valid method of identification by forensic experts and in court [103].

In the 1960s, law enforcement agencies began to develop automatic fingerprint identification systems (AFIS) in order to cope with the continuously growing database sizes and number of daily requests [13]. Nowadays, fingerprint recognition technology is also widely used in commercial applications, e.g. for access control to laptops or buildings, or for drawing money from ATM.

Despite all the advances in fingerprint recognition over more than a century, some fundamental questions have remained open.

\subsection{Thesis Contribution}

\subsubsection{Fingerprint Growth Analysis and Prediction}

How do fingers grow? The specific configuration of ridges is finalized after 24 weeks estimated gestational age [7] and there is a consensus about the permanence of the fingerprints' pattern, but the course of the growth process has, up 
to now, not been systematically examined. This lack of knowledge has practical consequences for law enforcement agencies as well as social implications.

Given a query fingerprint, the task of an AFIS is to find the corresponding finger, if it is stored in the database, and in this way identify the person to whom the query fingerprint belongs. If a person was registered to the database as a child or juvenile and the query fingerprint is acquired years later when the person is already a grown-up, then the AFIS is confronted with a major challenge. In this scenario, existing AFIS may fail to identify a person who is registered to the database, because the growth of the finger is not factored in adequately. Results presented in Section 2.4.4 give a first indication regarding missed identifications, but the exact number of failures that currently occur due to ignoring the impact of growth on fingerprints is unknown. This number can up to now simply not be counted, because from the perspective of the AFIS, the case of 'not in the database' and a missed identification are indistinguishable.

In the first part of the thesis, fingerprint growth is analyzed and a method for growth prediction based on the age of the person is proposed. Several tests demonstrate the effectiveness of the approach. After the implementation of these suggestions, law enforcement agencies can begin to estimate the dark figure of missed identifications by recording the cases in which the matching finger in the database was taken from a person under the age of 20 and determining the percentage of identifications that previously would have been missed.

Fingerprint growth is not only relevant for purposes of law enforcement and prosecution, but it also has societal significance due to the use of biometrics in visa applications and passports. In April 2009, the European Parliament and the Council of the European Union passed a resolution [1] regarding the introduction of biometrics in visa applications. The lack of knowledge regarding the growth of fingers caused the EP and the Council to exempt children under 12 from having their fingerprints included into the visa application. The according press release ${ }^{1}$ states:

During negotiations with the Council, MEPs ${ }^{2}$ successfully argued for children under twelve to be exempted from the requirement to give their fingerprints. At present there is no available large scale study that documents the reliability of juvenile fingerprinting. MEPs insisted on a prudent minimum age limit in order to ensure that the biometrics are reliable for the VIS to work as intended. On 14 January this year, the European Parliament voted for a similar exemption for biometric passports. The age limit will be re-examined in the light of the findings of the study that the Commission has undertaken to carry out at Parliament's request.

The conduction of a study analyzing the effects of growth on juveniles' fingerprints is enacted at the end of the resolution [1] and the first report is expected in 2012:

\footnotetext{
${ }^{1}$ http://www.europarl.europa.eu/sides/getDoc.do?pubRef=-//EP//TEXT+IMPRESS+20090324IPR52485+0+DOC+XML+V0//EN

${ }^{2}$ Members of the European Parliament
} 
The first report shall also address the issue of the sufficient reliability for identification and verification purposes of fingerprints of children under the age of 12 and, in particular, how fingerprints evolve with age, based on the results of a study carried out under the responsibility of the Commission.

The first part of the thesis contributes to reducing this gap in knowledge by analyzing the fingerprint growth of persons who were between 6 and 15 (median 11.9) years old at the time of their first check-out.

\subsubsection{Fingerprint Image Preprocessing and Multi-level Judg- ment Aggregation}

Individuality of fingerprints is commonly assumed by forensic experts, researchers and the general public, to the extent that the term 'fingerprint' has become a synonym for 'something that identifies; a trait, trace, or characteristic revealing origin or responsibility'3. On the other hand, fingerprint based identification is regularly challenged in court $[103,23]$ pleading that the uniqueness of fingerprints is not scientifically tested and matching error rates are not established. As a consequence, the 'Committee on Identifying the Needs of the Forensic Science Community' of the National Research Council, USA, reports [2] the need for strengthening the scientific foundation of fingerprint based identification.

Following the assumption that a fingerprint contains a sufficient amount of discriminative information, the challenge emerges how to reliably extract this information from a fingerprint image. The second part of the thesis addresses this pattern recognition challenge in the following way:

Chapter 4 treats the task of estimating the flow of ridges resulting in an orientation field (OF). Special regard is paid to low quality images in which large regions are disturbed by various kinds of noise aggravating the OF estimation. A review of relevant existing methods is given followed by the introduction of a novel OF estimation method called line sensor method which marks an improvement in comparison to state of the art methods. In Chapter 6, curved regions are presented as an approach of dealing with naturally occurring curvature of ridges in fingerprints. Subsequently, the curved regions are applied for improving the ridge frequency estimation. Chapter 7 surveys widely used fingerprint image enhancement techniques and introduces curved Gabor filters. This constitutes a synthesis of the previous two chapters, since the line sensor based OF estimation, the curved regions and the improved ridge frequency estimation method are combined.

Chapter 8 outlines the idea of multi-level judgment aggregation as an architecture for building an AFIS which makes good use of the plurality of existing methods on all levels of fingerprint image processing. Some first practical examples are presented and their performance improvements is validated in verification tests [73]. The results give a hint of the potential of this approach. Known fusion techniques are examined and discussed before a novel method of score

\footnotetext{
${ }^{3}$ Merriam-Webster's Collegiate Dictionary http://www.merriam-webster.com/
} 
revaluation is proposed. In a case study of FVC 2002 database 2 , the choice of appropriate score revaluation criteria is shown based on previously extracted information, in particular the orientation field. A commercial software is applied for minutiae extraction and matching which performs on the original images at an equal error rate (EER) of $1.08 \%$. In Section 8.8, all previously introduced methods for line sensor based OF estimation, RF estimation using curved regions, image enhancement by curved Gabor filters, template cross matching and score revaluation are united under the roof of multi-level judgment aggregation leading to a perfect, i.e. error-free separation into genuine and impostor recognition attempts. To the best of my knowledge, this is the first time that a perfect result was obtained on any of the FVC databases. Subsequently, the score revaluation criteria are generalized and tested on all available FVC databases. The thesis concludes with a discussion of the achieved results and possibilities for future work within the framework of multi-level judgment aggregation. 


\section{Part I}

\section{Fingerprint Growth}





\section{Chapter 2}

\section{Fingerprint Growth Analysis and Prediction}

\subsection{Introduction}

The dynamics of individual and average growth in humans have been extensively studied [53], particularly in terms of body height. But how do fingers grow? The question of finger growth is not only of theoretical interest in biology and auxology, but it has practical relevance for law enforcement agencies: if the person being checked out had been registered as a juvenile, retrieving a matching fingerprint in their databases poses serious difficulties to existing automated fingerprint identification systems (AFIS).

Studies of human growth show strong correlations between the increase in body height and the limb lengths (see e.g. [102]). Most studies concerned with the effects of growth on fingerprints have focused on the stability of the line pattern's structure. In 1892, Sir Francis Galton was among the first who noted the permanence of the configuration of individual ridges and furrows [42]. These findings were confirmed by biologists who discovered that the development of the pattern is finalized after 24 weeks estimated gestational age [7]. The deformation of this pattern due to the fingers' growth, however, received little attention [133]. One explanation why the effects of growth on fingerprints have not been investigated yet, may be the lack of longitudinal data of juveniles' fingerprints through adolescence. The data sets for the growth analysis were provided by the BKA (Bundeskriminalamt, Federal Criminal Police Office of Germany). The cooperation with the BKA offered a unique opportunity to work with data which is rarely available in other respects and the systematic examination of finger pad growth contributes to strengthen the scientific foundation of fingerprint recognition as requested by the National Research Council [2].

The fingerprint growth analysis and prediction presented in this first part of the thesis is joint work with Thomas Hotz who especially performed the statistical analysis of the data. The results can be found in a separate report [48]. 


\section{$2.2 \quad$ Data source}

The finger pad growth analysis is based on data sets of 48 persons (35 male, 13 female) provided by the BKA. During the initial enrollment of their fingerprints to the database, the subjects of this study were between 6.1 and 14.7 years old (median 11.9 years). At the time of final check out (CO), subjects were between 16.5 and 34.0 (median 24.5) years old. The images are scans of inked fingerprints on sheets of paper. Image resolution was 500 dots per inch. A full data set for a CO consists of 14 images: a nail-to-nail rolled fingerprint for each of the ten fingers and four additional plain control prints. One control print for each thumb and another control print for the other four fingers of each hand.

Each person was checked out in Criminal Records between 2 and 48 times (median 4.5 times). The whole data set consists of $367 \mathrm{COs}$. For the subsequent growth analysis and the test in Section 2.4.1, 8930 points of interest (POI) were marked. POI are minutiae, i.e. endings and bifurcations of ridges, and singular points, i.e. core and delta points. In order to investigate the growth effects, the locations of POI were determined in the fingerprint images of each person at all times of CO. Only in very few cases, POI could not be marked due to very low image quality. For each person, one finger was chosen for the analysis. The right index was selected, if the data set of a person contained one or more so called simplified COs which consist only of the right index finger. Otherwise, the most informative finger available was chosen for each person, i.e. the finger with the maximum number of unambiguously markable corresponding POI with respect to the image quality of overlapping areas. Fingers of the left or right hand are of tantamount eligibility, because differences in proportions between bones of the left and right body side have been found to be insignificant [120].

\subsection{Growth Analysis}

Naturally, the fingers' size will increase due to growth, but does it affect all of a fingerprint's parts similarly, or is there possibly a dominant direction, e.g. because fingers get rather elongated? If the latter was the case, distances along that direction would increase relative to distances orthogonal to it, requiring an elaborate correction step. In the analysis (see [48] for details), no dominant direction of variation or strong correlation with size was found. This suggests that growth has an isotropic effect on fingerprints; in other words, they get uniformly rescaled, and all that remains to be determined is the scale factor.

Taking the strong correlation of stature and limb lengths into account [102], we hypothesized this scale factor to be essentially the same for fingerprints as for stature. Determining the spread of minutiae in each fingerprint allowed us to obtain an intra-finger measure of size: we define size as the square root of the squared distances of the POI from their center of gravity. Out of all marked POI, only those corresponding pairs are considered which are shared at all times of $\mathrm{CO}$ (between 4 and 25 per person, median 11). We modeled the intra-finger size proportional to median stature as obtained from growth charts of boys and girls [88] and we found the model to explain the data very well with model misfit being smaller than variations at identical time points, the latter being caused by 

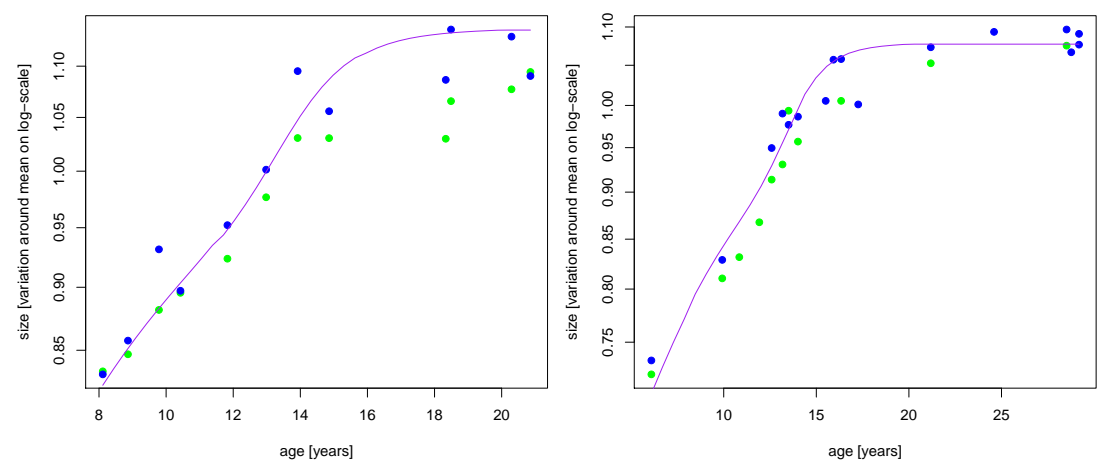

Figure 2.1: Mean distance of POI at different COs (blue: original, green: control) of persons 17 (left) and 31 (right), as well as fitted growth according to growth chart (purple) [48].

nonlinear distortions when the finger is pressed on a flat surface and systematic differences between originals and controls (see [48]). Another explanation for deviations from the median stature based model are individual characteristics such as varying times of on-set of the pubertal growth spurt.

Combining the strong correlation between finger pad growth and longitudinal growth with the isotropy results in a simple, practical method for predicting a fingerprint at a later stage in growth based on an earlier one: compute a scale factor as the ratio of median stature for the corresponding ages and gender and uniformly scale the earlier print up by that factor. The quality of this finger pad growth prediction is examined in the next section.

\subsection{Results of Validation Tests}

The validity and usefulness of the finger pad growth prediction is evaluated in light of the application we have in mind: matching a newly obtained fingerprint with a fingerprint in a database recorded several years ago before growth was completed. In order to obtain a broad understanding as well as practically relevant results, we performed several tests.

\subsubsection{Test 1: Minutiae Distances after Alignment}

For this test, the fingerprints of the first and last $\mathrm{CO}$ of each person are aligned using the POIs by choosing the combination of rotation and translation that minimizes the sum of the squared distances between pairs of POIs. Subsequently, the similarity of the two fingerprints was determined as the mean squared distance between corresponding POIs. This resulted in a measure of similarity where growth was not taken into account. Likewise, we took the accordingly rescaled fingerprints of the first $\mathrm{CO}$, aligning and comparing these to the fingerprints at the last CO, giving a measure of similarity after predicting growth as described above. In order to obtain a measure of dissimilarity due to non-growth related effects, i.e. chiefly nonlinear distortions of the skin when 

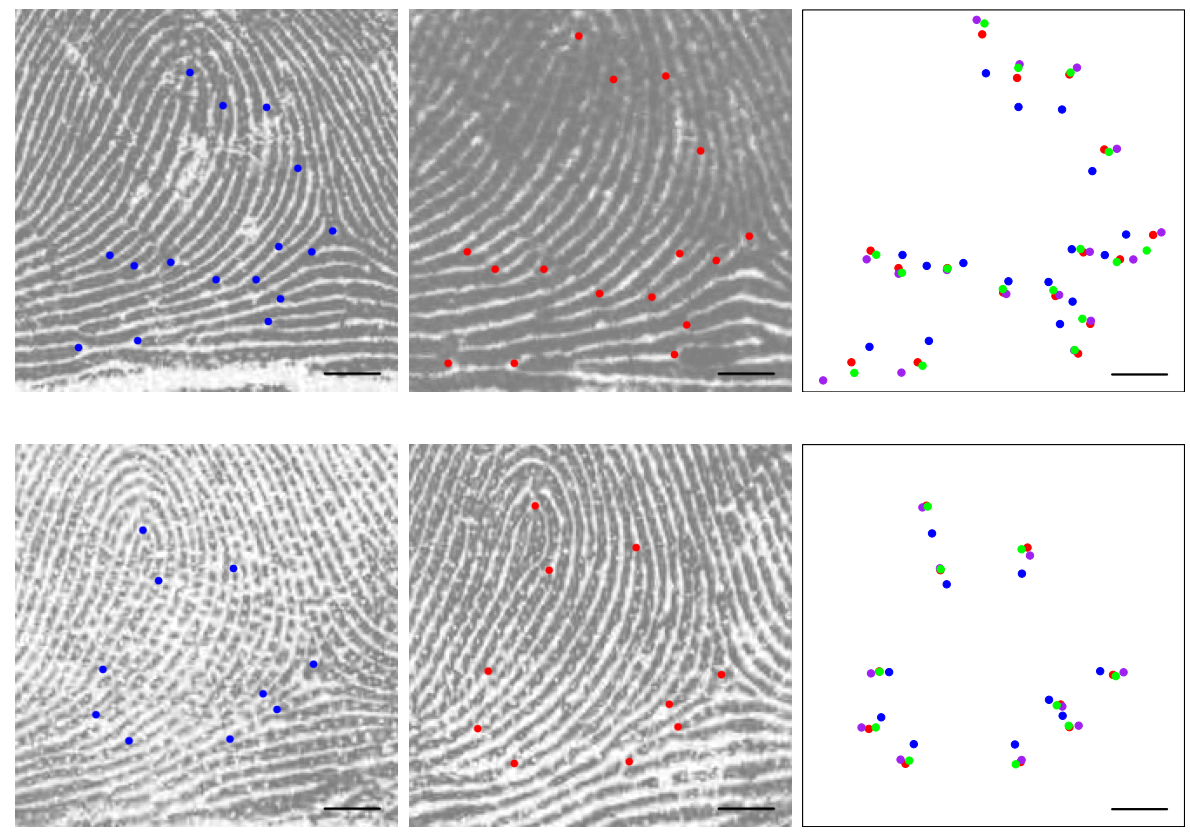

Figure 2.2: Imprints of right middle finger of persons 15 (top) and 18 (bottom) at first (left) and last (middle) CO with marked POI. Superimposed POI (right) at first $\mathrm{CO}$ without rescaling (blue), with rescaling (purple), each brought into optimal position w.r.t. the POI at last CO (red); control at last CO (green) shown for comparison (scale bars: $2 \mathrm{~mm}$ or 31.5 pixels) [48].

pressed on a flat surface, we also compared a second fingerprint recorded at the last $\mathrm{CO}$ for control purposes to the fingerprint at last CO.

Summarizing these intra-person measures by taking the median over our study population of 46 persons reveals that rescaling can reduce the median distance from 10.5 pixels when growth is not taken into account to 5.7 pixels with the suggested method. For comparison, the median distance between the two prints at the last $\mathrm{CO}$ was 4.7 pixels, the latter value being indicative of the achievable accuracy because these deviations in the relative location of the minutiae to each other are caused by the lack of reproducibility of the deformations that occur when an elastic three-dimensional object is pressed on a two-dimensional surface with variations in the angle, direction, speed and amount of pressure that is exerted. The corresponding empirical cumulative distribution functions comparing unscaled, first CO magnified and control print of the last $\mathrm{CO}$ are displayed in Figure 2.3.

Noteworthy, the distances between corresponding minutiae play an import role for the fingerprint matching algorithms which typically have a defined range of tolerance with respect to the amount of allowed fluctuation. For this reason, a gain in the matching performance by rescaling is to be expected, and accordingly, the second way in which we tested the validity of the growth prediction 

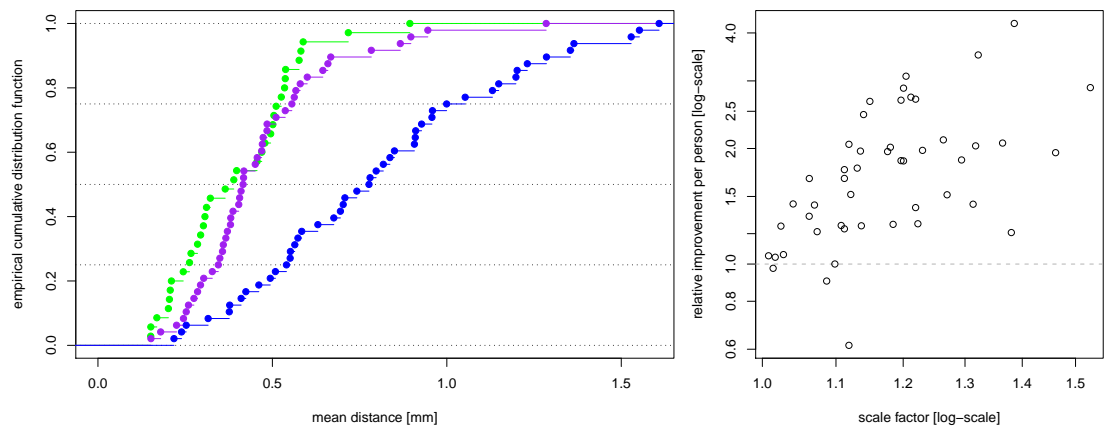

Figure 2.3: Left: cumulative distribution function of mean distance from POI at last $\mathrm{CO}$ after bringing $\mathrm{POI}$ at first $\mathrm{CO}$ without rescaling (blue) and with rescaling (purple) into optimal position, control at last CO (green) shown for comparison. Right: relative reduction in this mean distance per person achieved by rescaling vs scale factor given by the growth chart (numbers give person IDs) [48].

is by conducting several verification tests.

\subsubsection{Software for Minutiae Extraction and Matching}

Two software packages were used for the experimental results presented in the following section and the verification tests in Chapter 8 .

The first bundle is provided by the National Institute of Standards and Technology $^{1}$ (NIST) which is a agency of the United States Department of Commerce. It is called NBIS (NIST Biometric Image Software) and is freely available for download ${ }^{2}$. NBIS consists of a number of algorithms for fingerprint pattern classification, segmentation, quality estimation, minutiae extraction, matching and general purpose image utilities [144]. For this thesis, three NBIS algorithms were applied: MINDTCT for minutiae extraction, BOZORTH3 for matching and NFIQ [139] for global image quality estimation. MINDTCT extracts minutiae from a grayscale image and saves them in a text file (template). For each minutia, the $\mathrm{x}$ - and $\mathrm{y}$-coordinates, the direction $\theta$ and a quality indicator are stored. BOZORTH3 takes two templates as argument and calculates a similarity score. Henceforth the abbreviation BZ3 will denote results obtained using MINDTCT and BOZORTH3 without further options.

The second software package is called VeriFinger 5.0 Software Development Kit (SDK) provided by Neurotechnology ${ }^{3}$. It was chosen, because at the time of the experimentation, a license for the software was already available at our institute. Certainly, it is of interest to additionally conduct the verification tests, especially those described in Chapter 8, using further algorithms. VeriFinger was applied in two different ways using the functions VfeExtractUnpackedFromImage and

\footnotetext{
${ }^{1}$ http://www.nist.gov/

${ }^{2}$ http://fingerprint.nist.gov/NBIS/

3 http://www.neurotechnology.com/
} 
VfmVerify. Using the VeriFinger SDK, these two variants were created. The first takes two grayscale images as argument and outputs a score. It is labeled VFG for VeriFinger grayscale in the result tables. The second variant extracts minutiae from a grayscale image and stores the information in a template. For each minutia, the $\mathrm{x}$ - and $\mathrm{y}$-coordinates, the direction $\theta$ and the type (ending or bifurcation) are stored. In a second step, two templates are matched and a score is obtained, as well as additional information like e.g. which minutiae pairs were matched. The second variant is denoted as VFM for VeriFinger minutiae.

For the two minutiae based algorithms BZ3 and VFM, the rescaling was performed by adjusting the $\mathrm{x}$ - and $\mathrm{y}$-coordinates in the template files. For VFG, the grayscale images were rescaled and stored, and for the identification test conducted at the BKA using their in-house matcher, rescaling was performed by adjusting DPI settings.

\subsubsection{Test 2: Verification Tests}

Within the biometrics community, the most popular performance measures are the false rejection and false acceptance rates (FRR and FAR, respectively) in a verification test where a matcher must decide whether a query fingerprint belongs to the same person who had her fingerprint registered in a database: the database contains one fingerprint for each person, while the query set contains another fingerprint each. FAR and FRR are functionally related (see Figure 2.4 for receiver operating characteristic curves) via a threshold parameter that determines which scores, i.e. measures of confidence, produced by the matcher will lead to a claimed verification. In general, the smaller the FAR the larger the FRR. For comparing two matchers, one therefore often chooses the equal error rate (EER) [99], i.e. the rate obtained when the threshold parameter is selected in such a way that FAR and FRR become equal.

For our application, the query set is given by the fingerprints at the last CO. Similar to the analysis described earlier, we compare results for two different databases: one comprising the unaltered fingerprints at the first $\mathrm{CO}$, and the other one with those fingerprints rescaled according to their predicted growth. To ensure that the results do not depend on the particular matching algorithm used, three different matchers were applied as described previously.

On rescaling according to the growth prediction, there are two possibilities: the fingerprint (represented by a grayscale image or a minutiae template) of the first $\mathrm{CO}$ can be magnified (FM) according to the prediction or the last finger can be shrunken (LS) with the reciprocal value. The former may be of greater practical relevance because magnified templates can easily be kept in the database as a projection of the current fingerprint for persons who are presumably still growing. These templates should be regularly updated, in intervals that account for the speed of growth. A query against such a template would involve the same computational effort as a query against any other template, whereas shrinking the older, in most cases adult finger would require an individual rescaling for each combination of a query finger with a database finger of a person under the age of 20. In this section, the two rescaling variants FM and LS are compared with the unscaled (UN) images or templates. 

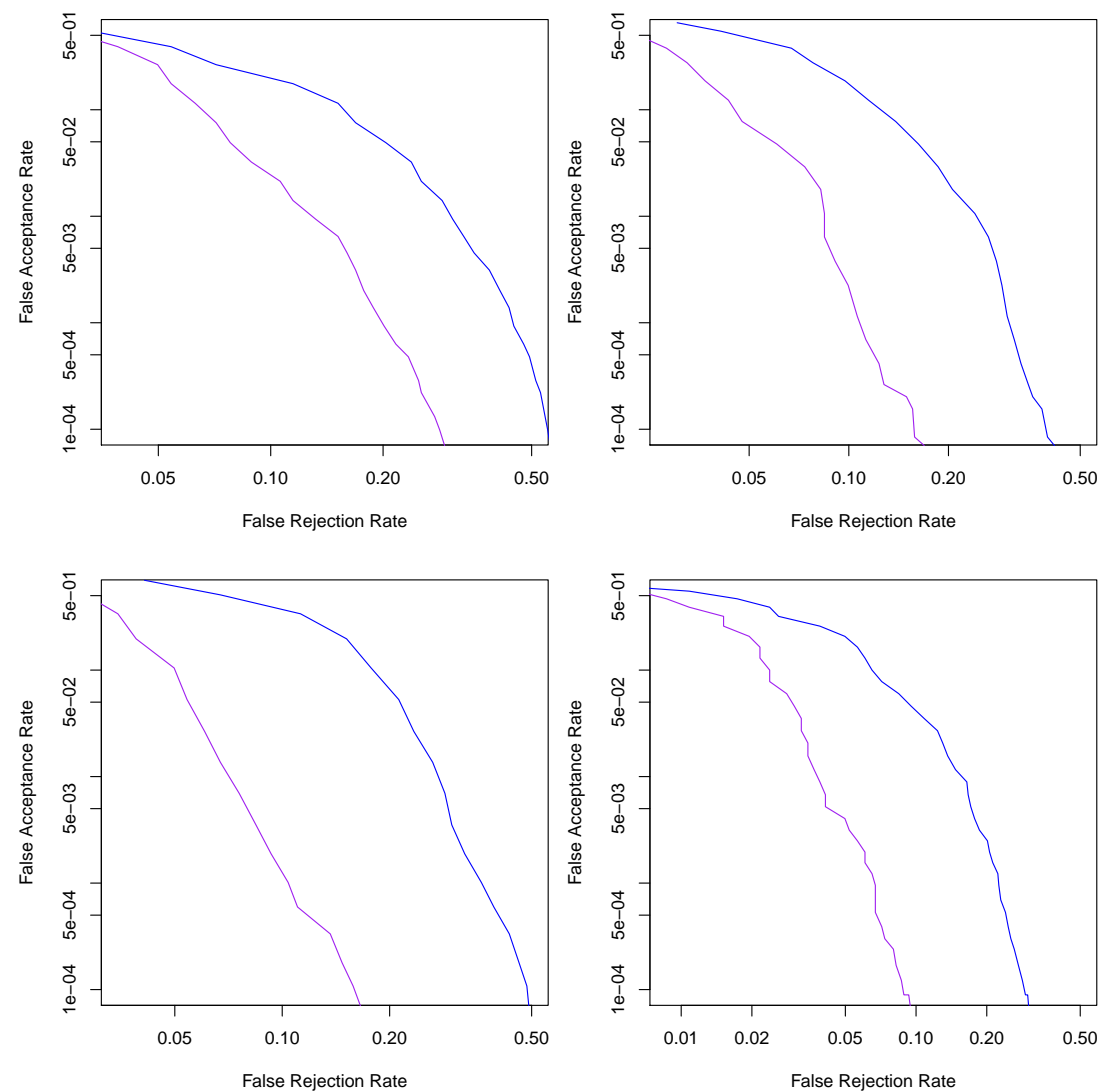

Figure 2.4: Receiver operating characteristic (ROC) curves comparing unscaled (blue) and first CO magnified (purple) for the algorithms BZ3 (top left), VFM (top right), VFG (bottom left) and score fusion of the three using the sume rule (bottom right).

The verification tests were conducted according to the following protocol: All available fingers of the first and the last $\mathrm{CO}$ are incorporated into the test. For 2 of the 48 persons, only the right index finger could be considered due to a so called simplified check-out, whereas for all other persons all ten fingers are included, resulting in 462 different fingers. For each finger of the last $\mathrm{CO}$, one genuine recognition attempt is performed by matching it against the same finger at the first $\mathrm{CO}$, and 461 impostor recognition attempts are conducted by matching it against all other fingers of the last $\mathrm{CO}$. This protocol corresponds to a scenario with a database containing a vast majority of adult fingerprints and only few prints of children and juveniles, as it is the case at the BKA. In order to measure the effects of rescaling, the performance in verification tests with three variants of genuine recognition attempts are compared: (i) the first and last $\mathrm{CO}$ of each finger are matched without scaling, (ii) the fingerprint of the first $\mathrm{CO}$ is magnified according to the growth prediction and matched against the unscaled print of the last $\mathrm{CO}$ and (iii) the fingerprint of the last $\mathrm{CO}$ is scaled 
down with the reciprocal value and matched against the unscaled first $\mathrm{CO}$.

In a first step, we compared the verification performance for each of the three matchers and the three versions of genuine recognition attempts (UN, FM and LS). The EERs for matching the unscaled fingers are located in the range from 10.81 to $14.11 \%$. EERs are cut in half by magnifying the first finger $(5.35-6.35$ $\%$ ) and reductions up to $77 \%$ are achieved by shrinking the last finger (3.28 $4.98 \%$ ). Score fusion using the sum rule (see also Chapter 8) leads to a further decrease of the EERs: UN 7.22 \%, FM 2.97 \%, LS $2.22 \%$ (13).

The growth prediction is based on the median body height charts for boys and girls [88] and it works well for the vast majority of examined cases. However, individual growth can deviate considerably from average growth. Genetic influence and environmental factors such as the nutritional status affect e.g. the timing of the pubertal growth spurt. In order to optimize the identification performance of an AFIS and include persons who grow considerably more or less than the median growth chart suggests, it stands to reason that matching with multiple rescaled versions of a finger results in higher matching scores for persons deviating from the median curve. For example, considering the two additional scenarios that a person grew $5 \%$ more or $5 \%$ less than predicted by the median curve and making use of the maximum score of these variations, leads to EERs in the range 1.98-5.05\% for FM and 2.19-2.36\% for LS. Score fusion using the sum rule achieves EERs of $1.76 \%$ (FM) and $0.9 \%(\mathrm{LS})$. The EERs can be even further reduced by considering more rescaling variants. With a combination of nine versions of rescaling and score fusion the EERs went down to $0.99 \%$ for magnifying the first finger and $0.37 \%$ for shrinking the last finger. The remaining matching errors were caused by very low quality of the fingerprints as found by visual inspection. The approach of using multiple rescaled fingers overcomes the difficulties of predicting individual growth from one single data set.

Theoretically, both scaling variants should perform equally well. In my opinion, the aforementioned differences in EERs between scaling up the first CO and scaling down the last $\mathrm{CO}$ are caused by specifics of the algorithms. Presumably, allowed ranges for matching minutiae are fixed, so that comparing two fingerprints of juveniles causes less false accepts, because the distance between matching minutiae are smaller than for the scaled up versions of the same images or templates. Once AFIS software designers begin to factor in the growth of fingers, these differences in performance should vanish. For practical reasons, the subsequently presented identification test at the BKA compares only the magnifying of the first $\mathrm{CO}$ to the unscaled version.

\subsubsection{Test 3: Identification Test at the BKA}

From a forensics point of view, verification is less of an issue. What matters more is identification [66]: whether a search in the database given some query fingerprint will return the same person's fingerprint as one of the top ranked results. For the purpose of a positive identification, a human expert will examine a small, variable number of potentially matching fingerprints depending on the score and factors like time resources and the importance of the case. In this 


\begin{tabular}{l|rr|rr} 
& \multicolumn{3}{|c}{ Top Rank } & \multicolumn{2}{c}{ One of Top 3 Ranks } \\
& unscaled & first CO magnified & unscaled & first CO magnified \\
\hline BZ3 & $59.1 \%$ & $82.5 \%$ & $67.5 \%$ & $85.9 \%$ \\
VFG & $64.3 \%$ & $90.5 \%$ & $69.5 \%$ & $92.4 \%$ \\
VFM & $69.7 \%$ & $90.7 \%$ & $75.3 \%$ & $92.0 \%$ \\
Score Fusion & $79.9 \%$ & $94.6 \%$ & $84.0 \%$ & $95.9 \%$ \\
3 Factors & $79.9 \%$ & $97.6 \%$ & $84.0 \%$ & $98.3 \%$ \\
BKA & $79.2 \%$ & $97.9 \%$ & $79.2 \%$ & $97.9 \%$
\end{tabular}

Table 2.1: Proportion of genuine matching scores ranked as the highest score or among the three highest scores, respectively.

study, the belonging of the genuine match to the top one or the top three search results are applied as criterion for measuring the success of the identification attempt.

The results of the verification tests could also be used as identification tests by determining how often the score of the genuine imprint at first $\mathrm{CO}$, unscaled or rescaled respectively, ranked among the corresponding impostor scores. We thus computed the respective proportion of genuines giving the highest score, or ranking among the top three (see Table 2.1). The identification test at the BKA was conducted on a database containing 3.25 million right index fingers, as well as a scaled and an unscaled version of the first check out of the 48 examined persons. Querying the database with the right index finger obtained at the final check out of each person, the unscaled data set was in 38 of 48 part of top three ranked results which means that if only the first $\mathrm{CO}$ were available, in 10 cases the person belonging to the queried finger could not be identified. For the data sets that were rescaled according to the growth prediction, the search was 47 out of 48 times successful. In one case, both versions failed due to low image quality. If the left index finger was used instead of the right one for that person, both versions could identify the person. Detailed results including the relative improvement of the matching score by rescaling can be found in [48].

\subsection{Anecdotes}

Remarkably, the statistical examination of the age and finger pad size data detected two clear outliers. Investigations by the BKA discovered that in one case the date of the check out in Criminal Records was mistaken by 10 years and for another person, the true year of birth is unknown. It would appear that the person might have stated a false year of birth in avoidance of punishment, and the finger pad growth data underpin this supposition. After the correction of these two entries, the outliers disappeared and the data sets were consistent with the growth model. 


\subsection{Discussion}

Drawing conclusions from this finger pad growth analysis yields simple, practical suggestions: law enforcement agencies can improve their identification performance by considering and predicting the growth of fingers for persons under the age of 20. Multiple rescaled templates account for deviations from the average growth and can easily be integrated into an existing AFIS. Regular (e.g. quarterly) database updates can adjust the fingerprint templates of juveniles according to the predicted growth. This procedure is technically feasible even for databases with millions of entries and the updates can be performed at times of low workload or off-line. The effectiveness of the prosed method for growth prediction was verified in three tests:

first, the minutiae distances after alignment of corresponding marked minutiae were dramatically reduced by rescaling. Second, the verification performance on the test set of 462 fingers was immensely improved and thirdly, this result result was confirmed on a BKA database consisting of 3.25 million right index fingers on which 9 failures to identify a person who was registered as a juvenile out of 48 identification attempts could be avoided by rescaling.

The exact number of failures to identify persons that currently occur due to ignoring the impact of growth on fingerprints is unknown. We learned of cases in which the records of the first and last $\mathrm{CO}$ could not be matched despite ten print matching. The identifications of these persons was only made possible by intermediate COs and chain inference. For these cases, the application of the growth prediction enables a successful matching. After the implementation of these suggestions, law enforcement agencies can begin to estimate the dark figure of missed identifications by recording the cases in which the matching finger in the database was taken from a person under the age of 20 and determining the percentage of identifications that previously would have been missed by the unscaled template. 


\section{Part II}

\section{Fingerprint Image Preprocessing}





\section{Chapter 3}

\section{Introduction}

The first stage of fingerprint image processing comprises of preparation steps which aim at improving the overall performance of the AFIS, i.e. minimizing the number of identification or verification errors, especially by enhancing the original input image. The importance of image preprocessing is illustrated by the fact that only very few fingerprint recognition algorithms abstain from preprocessing: e.g. a survey among the participants of the fingerprint verification competition "FVC 2004" [101] reveals that 25 of 29 participating algorithms segment the image at the beginning, and also 25 of 29 perform image enhancement before feature extraction and matching [18].

Fingerprint image processing includes methods and techniques for carrying out the following tasks:

- segmentation

- orientation field (OF) estimation

- singular point $(\mathrm{SP})$ detection

- classification

- image quality estimation (global or local)

- ridge frequency $(\mathrm{RF})$ estimation

- image enhancement

- binarization (e.g. [54])

A multitude of specific methods can be found in the fingerprint recognition literature for each of the listed tasks, and when one looks at the core ideas of the proposed methods, it becomes clear that aforementioned areas are deeply interwoven with each other. In all following chapters, this interconnection will be taken into consideration.

At the beginning of the next chapter, a review of existing methods for orientation field estimation is given and their advantages and limitations are discussed, especially when dealing with low quality images disturbed by typical types of 
noise. Having established the need for more robust approaches to OF estimation, the line sensor based method is presented and its performance is compared with two state of the art methods on artificially generated and naturally poor quality images. The interconnection of the line sensor method to segmentation and image quality estimation is addressed and it is sketched how these two tasks can performed simultaneously using the information obtained for the OF estimation. At the end of the chapter, the line sensor based method is further developed into a method which minimizes the computational efforts while preserving the robustness.

Chapter 5 sketches the application of quadratic Bezier curves for dealing with the naturally occurring curvature in fingerprints.

Chapter 6 discusses the relevance of ridge frequency estimation for fingerprint image preprocessing and gives a survey of existing methods for this task. The novel idea of curved regions is introduced which attempt to mimic the curvature of ridges and valleys by piecewise linear steps along the local orientation. The curved regions are applied in Chapter 6 for an improved method of ridge frequency estimation and in Chapter 7 for the design of a curved Gabor filter.

Finally, the concept of multi-level judgment aggregation is introduced in Chapter 8. A general architecture for designing better AFIS is recommended and some practical examples are given. All proposed methods for orientation field estimation, ridge frequency estimation and image enhancement from the previous Chapters $4-7$ are combined and in Section 8.3, the improvement of the verification performance applying this combined image preprocessing is reported. In the subsequent five sections, several approaches aiming at a further reduction of the error rates are investigated. Techniques known from the literature are examined, implemented and evaluated, and a novel idea for the post-matching stage called score revaluation is proposed which make good use of the information obtained at the preprocessing stage, in particular the orientation field. In the light of this information, score revaluation achieves a perfect separation of genuine and impostor recognition attempts in a case study of FVC 2002 database 2. To the best of my knowledge, this is the first time that an error-free verification result was achieved on any of the FVC databases. The subsequent generalization of the revaluation criteria leads to massive error reductions on all available databases. The chapter concludes with a discussion of the results and possible improvements. 


\section{Chapter 4}

\section{Orientation Field Estimation}

\subsection{Motivation}

A precise and reliable OF estimation is a crucial preprocessing step in fingerprint image processing, because many subsequently applied methods for ridge frequency estimation, image enhancement, binarization and feature extraction require information about the local ridge orientation, and computing singular points (SPs) using the Poincaré index [10] is based on the orientation field.

For example, if an image enhancement step using Gabor filters is incorporated into the automated fingerprint processing, this can improve the verification performance as e.g. shown in [60]. The Gabor filter is a local operator that takes the gray values of the image within a certain neighborhood, the local orientation and the local ridge frequency as input (as well as two additional tuning parameters: $\sigma_{x}$ and $\sigma_{y}$, the standard deviations of the Gaussian envelope. [103]). Applied with an accurate estimation of the local orientation and ridge frequency, the Gabor filter has the potential to significantly improve the image quality. On the other hand, if inaccuracies and mistakes occur during the OF estimation that can not be dealt with by techniques like low-pass filtering, incorrect local ridge orientations are subsequently passed to the Gabor filter. This will lead to artifacts (see e.g. [140]) in the enhanced image, especially to the creation of spurious bifurcations, and eventually decrease the matching results. The properties of Gabor filter are discussed in more detail in Chapter 7. For these and further reasons, the quality of the $\mathrm{OF}$ estimation can have a big influence on the overall performance.

\subsection{Review of Existing Methods}

\section{Gradients}

Currently, the most popular method for OF estimation is based on gradients and was introduced by Kass and Witkin in 1987 [82]. Its advantages are that it can be computed very fast, it performs well for many images, and unlike other 

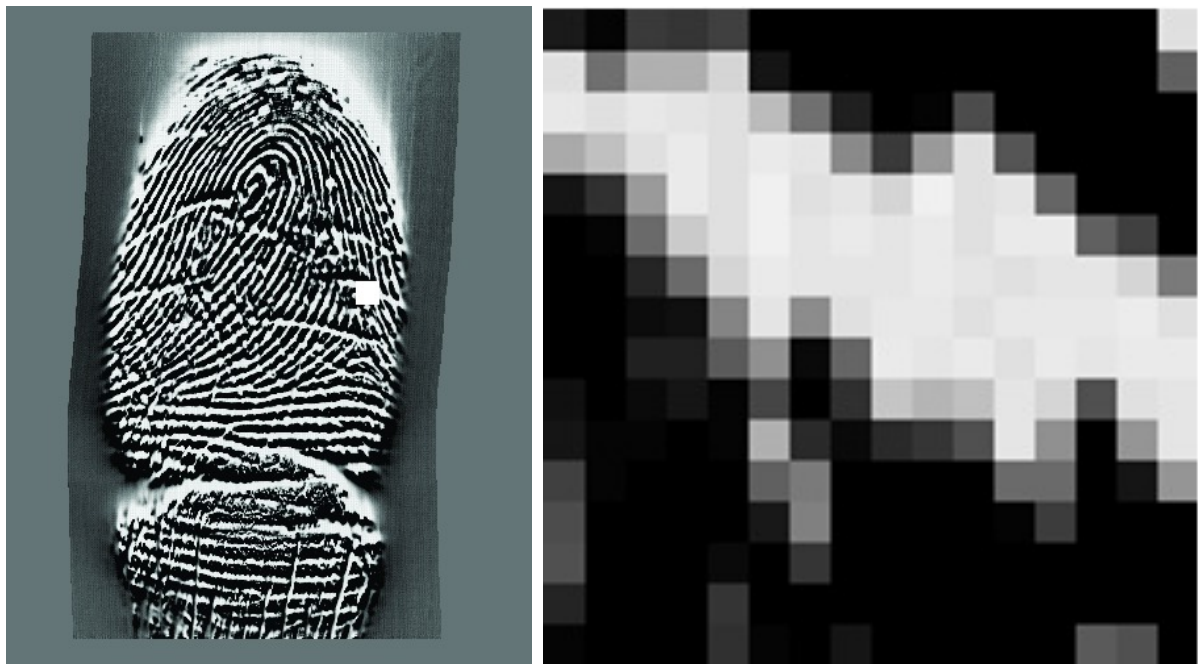

Figure 4.1: Image 1_10 from FVC2006 DB3 [17]: A $16 \times 16$ pixels detail (right) from the original image (left) in which the block is marked as a white square.

methods previously used, the accuracy of the calculated orientation is not limited by a fixed number of possible orientations [10]. Nevertheless, the gradients based method is susceptible to interference by scars, dirt, moisture or dryness of the finger, and other sources of noise which can lead to interrupted, thick or grainy ridge structures in the acquired image. The problem is inherent to the fact that gradients are extracted from the image using very small masks (of $3 \times 3$ up to $5 \times 5$ pixels). The results are then averaged over larger windows in order to increase the consistency of the results. Figure 4.1 (b) displays a $16 \times 16$ pixels detail from a fingerprint image that is disturbed by scar noise. In the depicted window, the orientation seemingly goes from the left top to the right center, but this detail belongs to an area disturbed by scars. For a human expert it is quite obvious, taking the surroundings of this window into account, that the true ridge flow roughly goes from top to bottom. The method based on averaging squared gradients fails to estimate the true ridge flow, because the information is not contained in this small window. Possible improvements rest upon neighboring windows that may catch a better orientation estimation, and on a subsequent smoothing step like low-pass filtering. A gradients based approach which combines Gaussian smoothed OFs at different scales was proposed by [11]. Since then, several additional methods have been proposed which are based on smoothing a coarse gradients derived OF at different scales (e.g., see [145],[107] and [80]).

\section{Normal Vectors}

An idea similar to the approach based on averaging squared gradients was presented by Donahue and Rokhlin [28]. They regarded a gray scale image as a surface over the xy-plane, calculated for each $2 \times 2$ pixels neighborhood the normal vectors and computed the orientation by a least squares minimization over the surface normals in an averaging window. This idea is related to the Marr- 
Hildreth operator [105] for edge detection which is also known as Laplacian of Gaussian (LoG) or Mexican hat, since the direction of the normal vectors is dictated by the gray-level intensity changes and peaks in the first directional derivative are equivalent to zero-crossings in the second directional derivative of intensity.

\section{Micropatterns}

Kawagoe and Tojo suggested a block wise OF estimation method [83] based on $2 \times 2$ pixels binary micropatterns. First, a fingerprint image is binarized and divided into subregions of $16 \times 16$ pixels. Each $2 \times 2$ pixels micropattern belongs to one of four classes representing the orientations of $0^{\circ}, 45^{\circ}, 90^{\circ}$ and $135^{\circ}$, or a neutral fifth class. For each block the occurrences of $2 \times 2$ pixels micropatterns are determined and compiled into a histogram of the four orientation classes from which an average block orientation is estimated.

A survey of further local methods for OF estimation can be found in [103].

\section{Global Models}

On the other side of the scale, global models strive to describe the whole orientation field by a number of parameters. Sherlock and Monroe [137] introduced a zero-pole global model using rational complex functions. An improvement by using a piecewise linear orientation function was proposed in [142]. The zeropole model [137] has been extended by Zhou and $\mathrm{Gu}$ [156] who suggested to approximate the rational function by a set of polynomials, and to use a pointcharge model in order to improve the accuracy in the proximity to singular points (see also $[157,51]$ ). For fingerprint matching, the usage of the globally modeled OF in combination with minutiae was proposed in [52]. The application of quadratic differentials for a global OF model was introduced in [64, 61]. All aforementioned global models have the disadvantage of requiring the prior knowledge of the singular points. First, a coarse gradients based OF is computed from which the type and location of the SPs is estimated. Inaccuracies and mistakes in this process lead to erroneous OF estimations by the global model.

Two models have been proposed that do not require prior knowledge of SPs: in [143], a model called FOMFE was presented that is based on 2D Fourier expansions in the phase plane and adapts directly to the coarse gradients based OF estimation. The second approach uses Legendre polynomials for smoothing the $\mathrm{OF}$ and correcting errors in the initial, gradients based estimation [126].

\section{Gabor Filter Bank}

The ridge and furrow pattern of a fingerprint can be regarded as an oriented texture [75] and the frequency- and orientation-selective properties of a Gabor filter [68] can be capitalized in a filter bank which chooses for each location the filter with the highest energy response and selects the frequency and the orientation accordingly. 


\section{D Sine Waves}

For the extraction of ridge patterns from palmprint images that are disturbed by many creases crossing the ridge lines, an approximation of the ridge patterns by 2 -dimensional sine waves was proposed in [40]. This method was refined in [69] by, amongst other things, a region growing algorithm that is similar to the one presented in Section 4.4.2.

\section{Multiscale Directional Operator}

A multiscale directional operator has been introduced by Oliveira and Leite [119]. It estimates the orientation of each pixel $(x, y)$ by dividing the semicircle into $D$ directions and calculates the standard deviation of the gray values for $n$ test points along each direction with $(x, y)$ as central point. From all pairs of two orthogonal directions, the pair exhibiting the highest information contrast is chosen. The information contrast is defined as the maximum of the absolute values of the differences in standard deviation between two orthogonal directions. Next, from the two directions of the chosen pair for pixel $(x, y)$, the direction with the smaller standard deviation is determined following the idea that the standard deviation along a ridge or valley is smaller than in the perpendicular direction, and this value is stored in a new orientation image $g$. Finally, the orientation at pixel $(x, y)$ is obtained by applying a smoothing window $\Omega$ centered in $(x, y)$ to image $g$.

The multiscale directional operator can be regarded as a generalization of Mehtre's idea for the computation of the directional image [106].

\section{Line Sensor}

The line-sensor based method which will be described in the following section traces ridge and valley lines and builds a coherent structure of locally parallel line segments from which the OF estimation is derived. To this end, the linesensor based approach makes good use of a property inherent in fingerprints: the continuity of ridge and valley flow perpendicular to the flow. This is a multiscale approach, since, at first, line segments are discovered locally. In a second step, neighboring parallel line segments will be merged and eventually they 'broadcast' their orientation to a medium-scale vicinity. By this means, the orientation field is constructed.

\subsection{Line Sensor Based OF Estimation}

\subsubsection{Sketch of the Method}

Firstly, the gray scale image is smoothed, binarized and morphologically improved. Secondly, a rudimentary line tracing detects ridges and valleys, completely or partially. Thirdly, the discovered line pieces are analyzed for parallel pieces in a neighborhood orthogonal to the line piece. Fourthly, we take the parallelism of line pieces into account and group them to parallel structures; the larger the structure, the higher the confidence in the resulting orientation estimation. All structures that cover a minimum number of pixels are merged, 
and all line pieces propagate their orientation orthogonally. In this way, the orientation field is estimated. Finally, missing blocks are iteratively reconstructed until the orientation field is complete. See Figure 4.7 for an illustration of the main steps.

\subsubsection{Results}
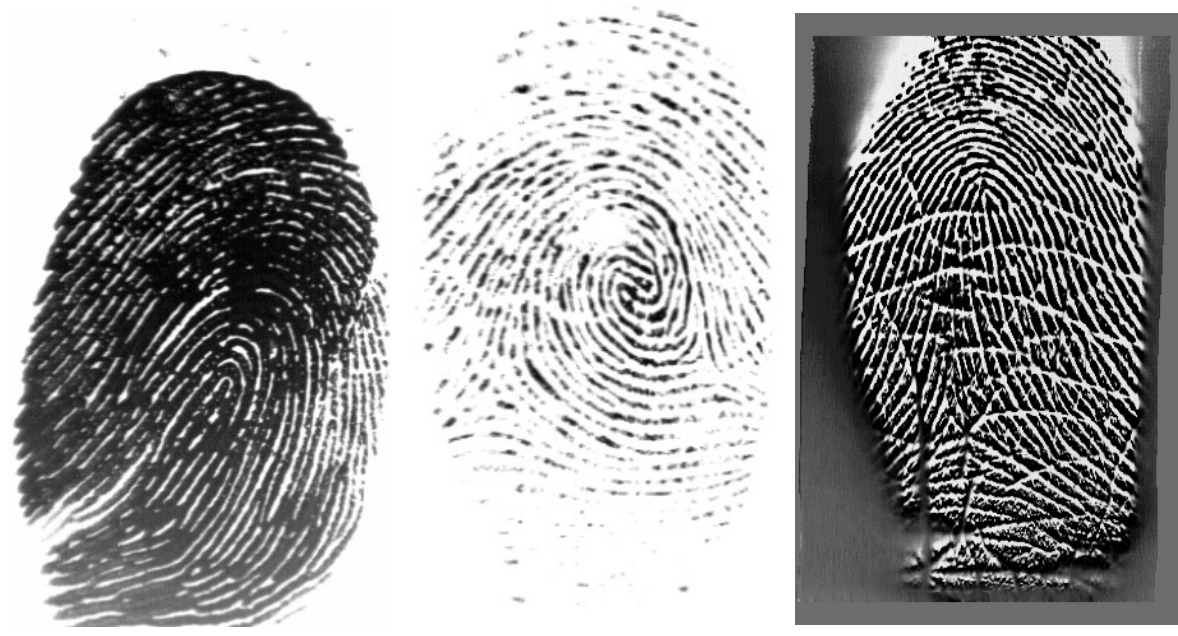

Figure 4.2: Examples of three typical types of noise from FVC2004 [101]: a wet (left, image 108_8 DB1) and a dry fingerprint (center, image 1_7 DB1). The fingerprint on the right (image 10_1 DB3) is disturbed by scars.

In order to investigate the performance of our method, in a first step we chose a sample of good quality fingerprint images from the FVC2000 [99] and FVC2002 [100] databases. The images were divided into blocks of $16 \times 16$ pixels. A human expert classified the blocks as foreground or background and marked the orientation of all foreground blocks. Next, artificial scars were simulated by drawing white bars on top the good quality images. We compared the OF estimations of the line-sensor based method, the gradients based method [10] and the multiscale directional operator [119] to the OF marked by the human expert. As performance measure, the number of deviations $>15^{\circ}$ between an estimated orientation and the marked 'ground truth' orientation was used. For the gradients, averaging window sizes from 8 to 100 pixels were applied, and for the multiscale directional operator, 36 combinations of the three parameters number of directions, number of test points and smoothing window size were computed.

For the original good quality images, the gradients based method and the linesensor based method achieved the best results with 12 deviations $>15^{\circ}$ out of 4072 foreground blocks $(0.29 \%)$ and 20 deviations $>15^{\circ}(0.49 \%)$, respectively. The multiscale directional operator produced 105 erroneous estimations $(2.58 \%)$. With an increasing number of simulated scars, the performance of the gradient based method deteriorates proportionally to the amount of added scar noise, whereas the line-sensor based method shows greater robustness to 
this type of simulated noise. The number of defective estimations by the multiscale directional operator increases slowly in comparison to the gradients based method, but its performance is clearly worse than the line-sensor based method (see Figure 4.11).

In the main part of the experimentation, 1782 orientations were manually marked in 60 poor quality images from FVC2004 [101] representing three typical types of real life noise: wet finger, dry finger and finger with scars (see Figure 4.2). For the convenience of the reader, all marked orientations are made available ${ }^{1}$ for comparative tests. On these naturally poor quality images, the performance of the three methods was compared using the same measure.

The line-sensor based method clearly outperformed the other two methods on these naturally poor fingerprint images. Using the number of deviations $>15^{\circ}$ between the estimated and the marked orientation as performance measure, the gradients based method produced $50 \%$ more defective orientation estimations than the line-sensor based method, and the multiscale directional operator produced more than three times the number of errors. On the other hand, the presented method has difficulties in the core area, where due to the ridge bending the approach based on parallel traced segments has its limitations, and the computational costs for the line-sensor based OF estimation are greater than the requirements of the gradient based method.

\subsection{Detailed Description of the Line Sensor}

The various steps of the algorithm described below depend on a variety of significant constants. Large variations of the values of these parameters are conceivable. We have chosen these values empirically, striving to optimize the estimation performance.

\subsubsection{Preprocessing}

\section{Binarization}

Goal of the binarization is to prepare the subsequent line tracing by obtaining a binary image with a ridge and valley structure that is as distinct as possible with respect to the local noise level. This is achieved in three steps: firstly, the gray scale image is smoothed by a two-dimensional Gaussian filter [135]. Secondly, it is binarized by applying a basic adaptive mean threshold [138] and finally single pixels and very small 4-connected pixel areas are removed using a seed fill method [55].

In the tests with images from all FVC databases and different combinations of parameters, the following produced the best results: a Gaussian filter with mask size $7 \times 7$ pixels and $\sigma=1.0$, a $5 \times 5$ pixels window for the locally adaptive mean threshold and the removal of all 4 -connected areas $\leq 10$ pixels.

\footnotetext{
${ }^{1}$ Please refer to the website of the biometrics working group at the Institute for Mathematical Stochastics, Georg-August-University Goettingen: http://www.stochastik.math.unigoettingen.de/biometrics/
} 

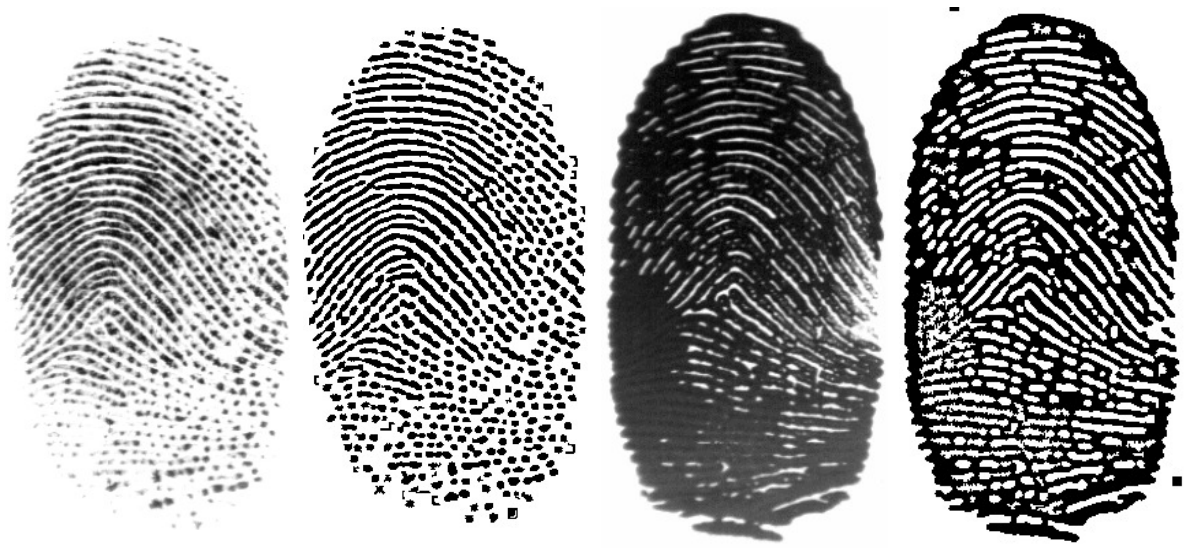

Figure 4.3: Original grayscale and binarized images 101_6 (left) and 101_8 from FVC2004 DB1 [101].

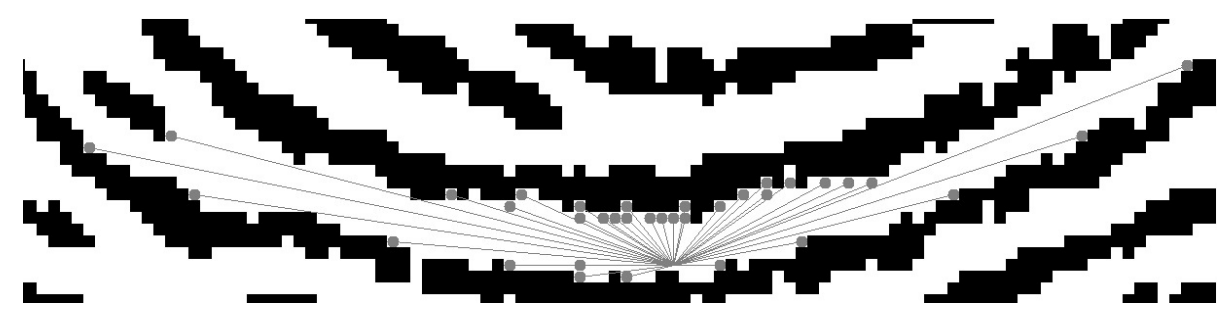

Figure 4.4: The sensor peers from a start point into a circular set of directions.

\section{Tracing}

The line tracing is based upon the principles of the sensor-like 'Entracer' introduced in [110]. Lines are traced in black (ridges) and white (valleys). The tracing of valleys is necessary in binarized images of dry fingerprints, whereas ridge tracing is appropriate for wet prints (see Figure 4.3). It is desirable to trace complete ridge and valley lines in order to retrieve as many pieces of information as possible, but for the purpose of orientation field estimation, it is sufficient if only some parts of lines are traced.

For choosing start points, we iterate in a grid over the binarized image. Initially, all grid points are permitted as start points and marked accordingly in a two-dimensional Boolean array. After a line was traced, grid points which are part of the line and neighboring pixels of the same color in the binary image are excluded from being start points. In this way, double tracing of lines is avoided. On commencing to trace a line, the sensor peers from the start point $p_{0} \in B(i, j)$ into $d$ directions as shown in Figure 4.4. Here, $B(i, j)$ denotes the $(i, j)$-th pixel of the binary image $B$. For each direction, the number $n$ of possible pixel steps in the same color are measured. After sorting, let $n_{0}$ be the biggest value. Prerequisites for beginning to follow a line: firstly, $n_{0}$ must be greater than a threshold value $t_{\text {minStartLen }}$, and secondly, all other directions with $n_{i} \geq t_{\text {minStartLen }}$ must be situated in a cone around the direction belong- 


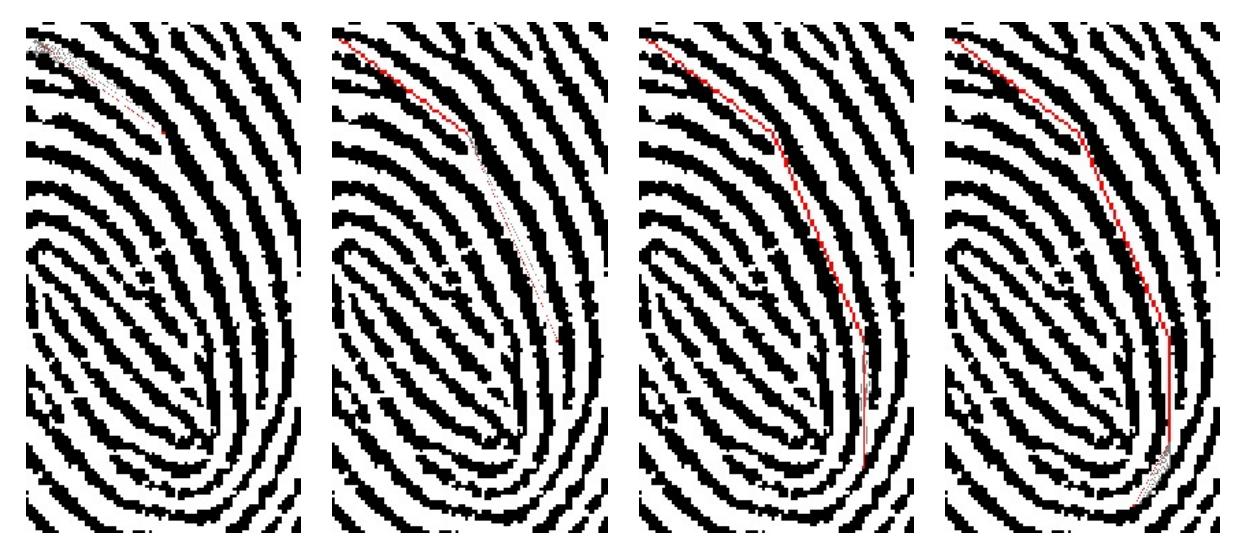

Figure 4.5: Starting in the top left corner of the first image (left), the line-sensor performs an all-around search and traces the line into the direction which can be followed for the longest possible distance (red line). From the next point on, the line-sensor explores only those directions that are located in cone around the recently followed direction (second image).

ing to $n_{0}$ or in a cone around the opposite direction. If there are other directions with $n_{i} \geq t_{\text {minStartLen }}$ outside these two cones, the tracing is aborted, because the start point might be part of the image background or an area disturbed by noise. If this initial all-around search detects exactly one or two directions, the tracing begins. The second direction is the one belonging to the biggest $n_{i}$ in the opposite cone. Now, the line is followed $k \leq n_{0}$ pixel steps into the first direction to the point $p_{1} \in B(i, j)$. The choice of each $k$ aims at going as far as possible in one direction and simultaneously ensuring that the next point is close to the center of the followed line for being able to continue the tracing. Now looking from point $p_{1}$, the sensor searches in the cone around the followed direction for the next possible pixel steps without changing the color (see Figure 4.5). The angle of the cone shall not be much larger than 'normal' ridge bending. Let $n_{0}$ be the biggest pixel step value and $t_{\text {minContinueLen }}$ the minimum length necessary for the continuation of the tracing. If $n_{0} \geq t_{m i n C o n t i n u e L e n}$, the line is followed in the direction which belongs to $n_{0}$ and this step is iterated. Otherwise the line is terminated and if there is a second direction at the start point, the line is tracked in that direction in the same manner. Also, after each step from $p_{k}$ to $p_{k+1}$, this piece is marked as an 8-connected line in the start point image and it is checked whether an already discovered line was crossed. If so, the intersecting angle $\alpha$ is determined. For an $\alpha$ smaller than a threshold $t_{\alpha}$, the tracing is stopped in order to avoid the double tracing of lines, for a big $\alpha$, the line tracing continues, and the decision, which of the two lines expresses the true ridge flow and which line tracks a scar, will be postponed. After a line can not be traced further, it is stored in a list. The color of the line and intermediate points are stored. Henceforward, 'line piece' or 'line segment' denotes the segment between of two consecutive intermediate points on a traced ridge or valley.

For the experimentation in Section 4.5, the following parameters were used:

- grid size $g=4$ pixels 


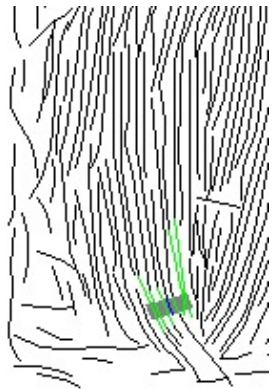

Step 1

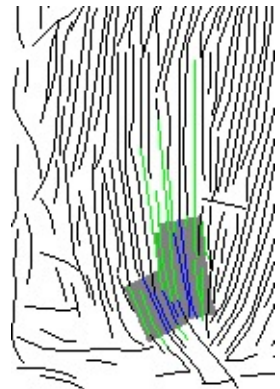

Step 2

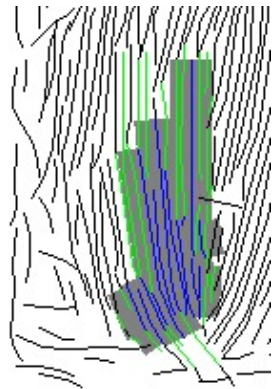

Step 3

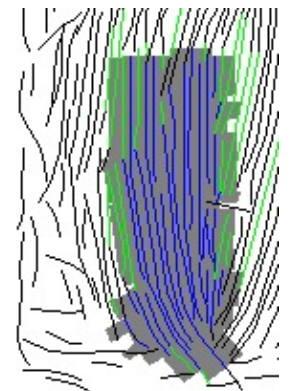

Step 4

Figure 4.6: In step 1, the gray polygon (range $=24$ pixels) covers the proximity of the blue line piece in orthogonal direction. Other line pieces (traced ridges or valleys) crossing the polygon are examined for whether they run parallel (green), i.e. forming an angle $\leq 7.5^{\circ}$ with the blue piece. The ratio of green pixels within the polygon to the total number of polygon pixels is 0.21 in this example. Discovered parallel pieces (green) in step 1 are added to the structure. In step 2, the grown structure of coherent pieces is drawn in blue, and for each newly added piece the corresponding polygon (gray) is inspected for crossing line pieces. The iteration continues as long as new parallel pieces are discovered.

- number of directions $d=120$

- minimum start length $t_{\text {minStartLen }}=15$ pixels

- minimum length for continuing the tracing $t_{\text {minContinueLen }}=5$ pixels

- $\operatorname{start}$ cone size cone $_{\text {start }}=45^{\circ}$

- $\max$ bending threshold cone $_{\text {bending }}=15^{\circ}$

- intersecting angle threshold $t_{\alpha}=22.5^{\circ}$

Related work: For the purpose of minutiae detection, a different tracing approach operating in the grayscale image was proposed by Maio and Maltoni [98]. Extensions are given in [94] by tracing both, ridges and valleys, and in [79] by adaptive tracing and filtering.

\subsubsection{Orientation Field Estimation}

\section{Evaluation of Traced Lines}

Once, a list of lines consisting of straight pieces was compiled, the critical question arises: which lines, or which pieces of lines, reflect the true ridge flow, and which pieces belong to traced scars and other sources of noise?

Bearing in mind the continuity of ridge flow perpendicular to the flow direction, such line pieces tend to comprise the true ridge flow that are confirmed by many parallel line pieces. Traced scars may also have other parallel scar pieces, but from a broader perspective, they remain a local phenomenon. The number of parallel ridge and valley line pieces are detected for each line piece in the 
following way: A vector orthogonal to the piece's orientation is added to and subtracted from the start point and the end point of the piece. These resulting four points form a polygon which covers the neighborhood in orthogonal direction. It is examined whether the pieces crossing this polygon run parallel (see Figure 4.6 step 1). If so, simply the number of pixels that belong to the parallel piece and are located inside the polygon are summed up. The ratio of parallel pixels to total pixels of the polygon is used as an indicator for the goodness of the piece. This ratio and the polygon are stored for each piece and they will be utilized in the next step. A good line is characterized by the fact that a high percentage of lines and line pieces in the region surrounding the good line run parallel. This, to some extent, is also true for lines that are very closely located to the core and delta points.

\section{Growing Coherent Structure}

After sorting all line pieces according to descending parallel ratios, let $p_{0}$ be the piece with the biggest proportion of parallel parts. Beginning with $p_{0}$ and a new, blank image coherent structure $(C S)$, a structure of coherent pieces is created as follows: $p_{0}$ is added to a new list $s 1$ which keeps track of all pieces building this structure. The polygon belonging to $p_{0}$ is drawn on $C S$ and in the start point image $S$, where all line pieces are marked, the pieces located in or crossing the polygon area are detected (see Figure 4.6). If a piece under consideration and $p_{0}$ form an angle $\alpha \leq t_{\text {parallel }}$, the new piece is classified as parallel and added to the list $s 1$. Next, for each piece $p_{k}$ that was added during the last step, the according polygon is drawn on $C S$ and that area of the polygon which was previously blank is checked in image $S$ for the occurrence of pieces. Each detected piece parallel to $p_{k}$ is appended to the structure $s 1$. The iteration lasts until in a single step no new parallel pieces are discovered and the growth of structure $s 1$ stops. The pixel count of the whole structure is stored. From the list of line pieces, sorted in descending order with respect to their ratio, the next item is analyzed. If piece $p_{1}$ is not part of structure $s 1$, a new structure $s 2$ is built starting with piece $p_{1}$, else it is continued with piece $p_{2}$.

Finally, all structures that cover an area of more than $t_{\text {minPixel }}$ are merged. Traced scar lines may have some parallel pieces and grow to a structure, but even in the case of two or more parallel scars this remains a local phenomenon, and in this merging step all possible scar influence is excluded. For the experimentation in Section 4.5, the parameter was chosen: $t_{\text {minPixel }}=1000$.

Related work: A region growing algorithm proposed by Jain and Feng [69] for blocks of 2D sine waves instead of line pieces rests upon a similar philosophy. Another idea for verifying thinned ridges extracted from a binarized image was presented in [59] for OF estimation and subsequent fingerprint classification.

Two papers were recently published which especially address the problem of creases and scars: Oliveira and Leite [119] propose to detect and reconnect broken ridges using a multiscale directional operator in combination with morphological tools. In [155] creases are detected for removing spurious minutiae and as a feature for fingerprint matching. 

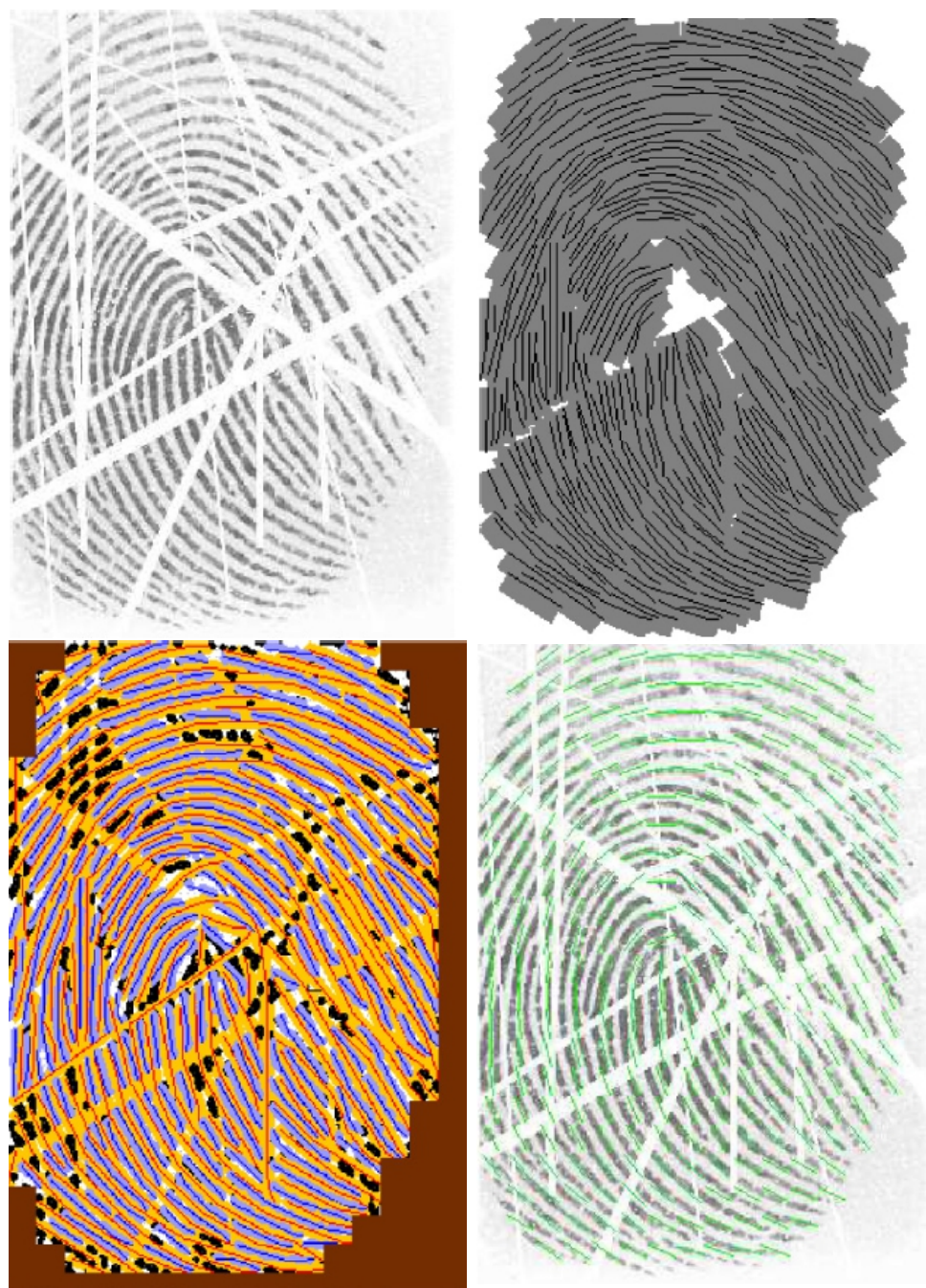

Figure 4.7: The main steps of the line-sensor based method. Top left: image 107_7 from FVC2000 DB2 [99] with 15 randomly added artificial scars; top right: traced ridges (blue) and valleys (red); bottom left: merged coherent structure; bottom right: derived orientation field after reconstruction. 


\section{Orientation Field Estimation}

For the orientation field estimation, the image $I$ is divided into blocks. Reasonable values for the block size $b$ are in the range from 1-16 pixels. For the scar simulation in Section 4.5.2, a block size of $b=8$ pixels was chosen. Each block $B(a, b)$ is initialized with a vector $v(a, b)=[0.0,0.0]$. Iterating over the merged line piece structure created in the last step, each line piece propagates its orientation to the $\mathrm{OF}$ in the following way: The polygon belonging to the line piece is reused for determining which OF blocks are overlapped by it and how many pixels are covered. Let $\phi_{k}$ be the orientation of line piece $p_{k}$ and assume it covers $n_{k,(a, b)}$ pixels of block $B(a, b)$. Then, the weighted influence of piece $p_{k}$ on block $B(a, b)$ is $w_{k,(a, b)}=n_{k,(a, b)} / b^{2}$ and $v(a, b)$ is updated: $v_{\text {new }}(a, b)=v_{\text {old }}(a, b)+\left[w_{k,(a, b)} \cdot \cos \left(2 * \phi_{k}\right), w_{k,(a, b)} \cdot \sin \left(2 * \phi_{k}\right)\right]$.

The idea of doubling the angles before summing up the vectors was introduced by Kass and Witkin [82]; it reflects the fact that tangents to flow lines have no direction, so the angles are defined $(\bmod \pi)$. The orientation of each block is calculated by $\phi(a, b)=0.5 \cdot \operatorname{atan} 2(v y(a, b), v x(a, b)) \bmod \pi$ [103]. This propagation makes good use of the continuity of ridge flow orthogonal to the flow direction which is an intrinsic property of fingerprints. Finally, the polygons belonging to the line pieces of the merged coherent structure are reused for deciding which blocks of the orientation field receive enough orientation influence by the line pieces. If the original image is disturbed and has some noisy areas, this tends to lead to hiatuses in the merged coherent structure. Now, these missing blocks are determined and they will be reconstructed in the next step. Starting with a blank image $P$, all polygons appertaining to the line pieces of the merged coherent structure are drawn on $P$. For all blocks that are nearly completely or totally covered by polygons, the orientation $\phi(a, b)$ is stored, all other blocks are marked as missing.

\section{Reconstructing Missing Blocks}

First, it is determined which missing blocks are 4-connected with each other and together, they form a gap in the OF. It is checked whether a gap adjoins the image border or not, and the information is stored.

\section{Small Gaps}

Single or two connected missing blocks are simply reconstructed as follows: The missing block is regarded as the center of a $5 \times 5$ blocks neighborhood. Let $\left(x_{m}, y_{m}\right)$ be the coordinates of the missing blocks center, $\left(x_{a b}, y_{a b}\right)$ of block $B(a, b)$ respectively. Let $\alpha_{(a b), m}$ be the angle between the orientation of block $B(a, b)$ and the line from $\left(x_{a b}, y_{a b}\right)$ to $\left(x_{m}, y_{m}\right)$ with $0 \leq \alpha_{(a b), m} \leq \pi / 2$ and $d_{(a b), m}$ be the Euclidean distance. The influence of each block is weighted with $w_{a b}=\left(2 * \alpha_{(a b), m}\right) /\left(\pi *\left(1+d_{(a b), m}\right)\right)$. Thus, blocks close to the missing block have a greater influence than those further away, and blocks with an orientation parallel to the missing block's location are weighted stronger. For an example, see Figure 4.8. 

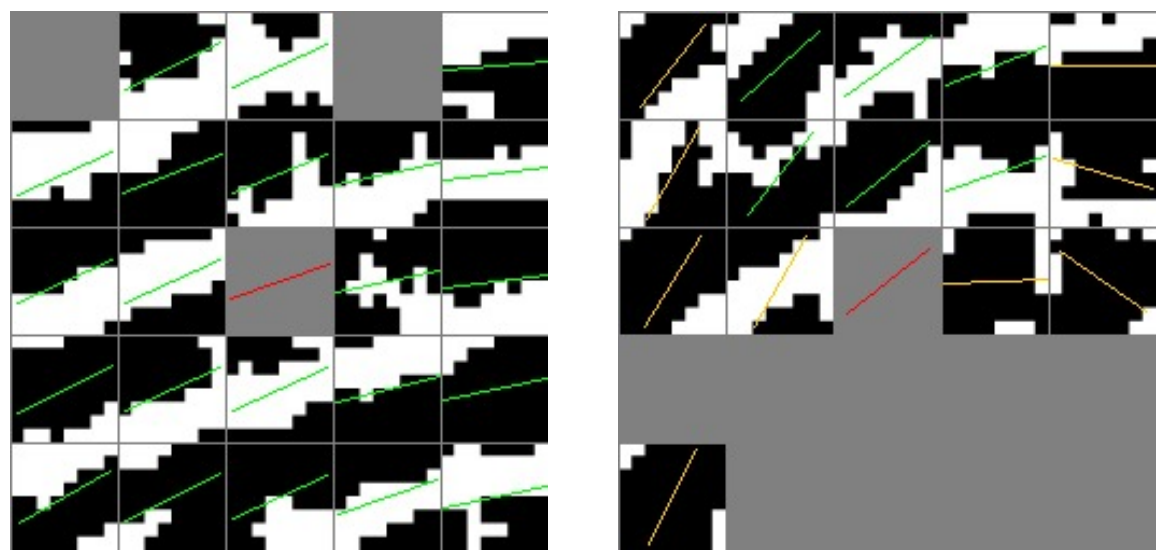

Figure 4.8: The central block is reconstructed, missing blocks are gray. On the right, an area close to the core is reconstructed and only the orientations in green influence the estimation, the orange ones are ignored.

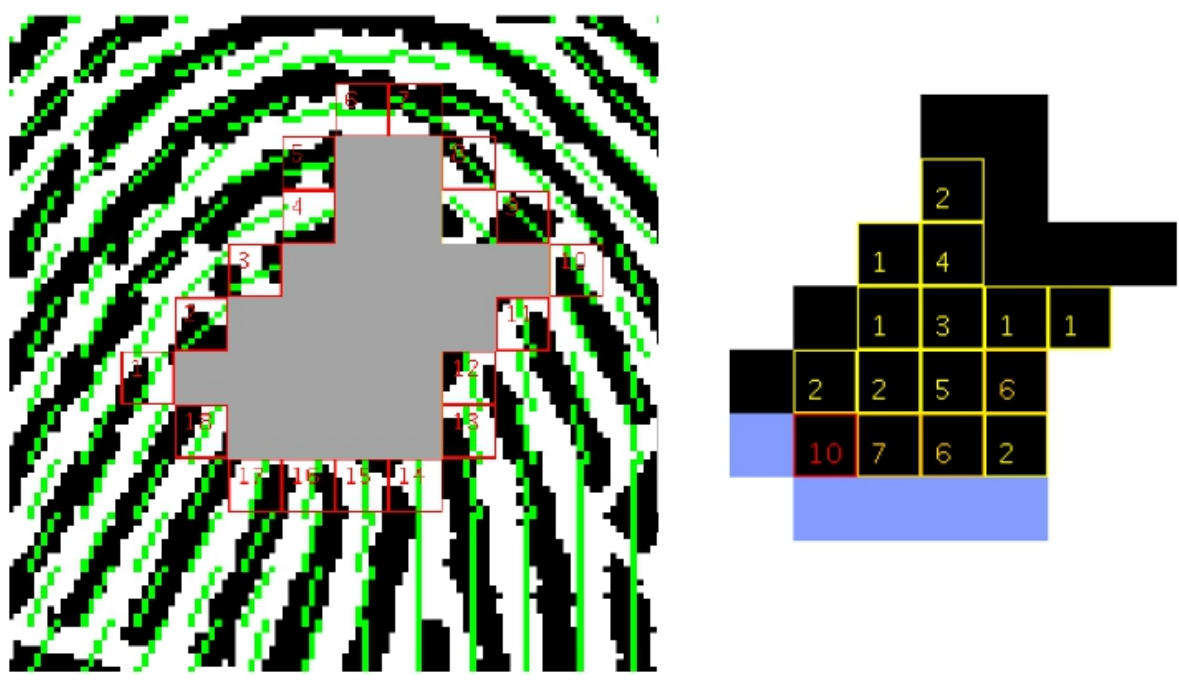

Figure 4.9: On the left, the Poincaré index is calculated using the margin of the big gap. On the right, the numbers of intersection points within each block are displayed.

\section{Big Gaps}

For three or more missing blocks connected with each other, a path on the margin around the big orientation field gap is detected and the Poincare index is calculated. If the result is 0 , then the area contains no singular points, and it is reconstructed like the single block previously, starting with the missing block that has the largest weights from the neighboring blocks. The same procedure is applied for gaps containing a delta.

If the Poincaré index indicates a core or a whorl inside the gap, the location of 
the core point is estimated by the intersection points of lines perpendicular to the orientation as proposed by Novikov and Kot [117]. From each block along the margin, the line perpendicular to its orientation is drawn and intersections are counted. Figure 4.9 shows the numbers of intersection points. Blocks whose normal lines did not cut the gap are marked in blue. The blue blocks in Figure 4.9 correspond to the margin blocks 14 to 18 in Figure 4.9. In the case of more than one candidate for the block containing the core point, the block with the smallest average distance to the blue blocks is chosen. After the core block is determined, the reconstruction begins with the missing block that is farthest away from the core. Unlike before, only those neighboring blocks are accounted for that are located in the cone around the direction from the core block to the missing block (a cone of $15^{\circ}$ was used), and that are further away from the core than the missing block (see Figure 4.8). Orientations of reconstructed blocks are included in subsequent reconstruction steps. See Figure 4.10 for a successful reconstruction of a whorl (left column) and an example in which the reconstruction fails.

\section{Border region}

Having finished the reconstruction of all missing blocks inside the orientation field, the OF estimation is gradually expanded from the center to the image border in the same way as the single blocks were reconstructed. This proceeds until the orientation of all blocks is estimated.

\subsubsection{Segmentation and Image Quality}

A byproduct of the growing coherent structure in Section 4.4.2 is the classification of the area covered by this merged structure as foreground. For an illustration, see Figure 4.7 image (d). The union of all polygons belonging to the line pieces of the merged coherent structure form the gray area and can be regarded as foreground. Small gaps can easily be filled by applying the composite morphological operator 'closing' [65].

Furthermore, a local quality index can be intuitively derived from the coherent structure in combination with the traced line segments that are located in the foreground but are not part of the coherent structure by computing the relation of 'good' line segments which are part of the coherent structure to the overall number of traced line segments in that area. An exception is the direct neighborhood to core points where due to the high curvature it tends to be difficult to find parallel pieces, In the terminology of [4], the aforementioned quality index belongs to the category of measures which are based on the local direction. At the matching stage, the local quality index can be used for evaluating the reliability of the extracted features so that features from high quality areas gain more influence [22].

Also, this quality index can be mapped easily to the division of the image by Hong et al. [60] into the categories 'well-defined', 'recoverable' and 'unrecoverable'. In our model, a well-defined area consists mostly of line segments that are part of the coherent structure of parallel pieces, whereas a neighborhood which is covered by the coherent structure and simultaneous has a considerable 

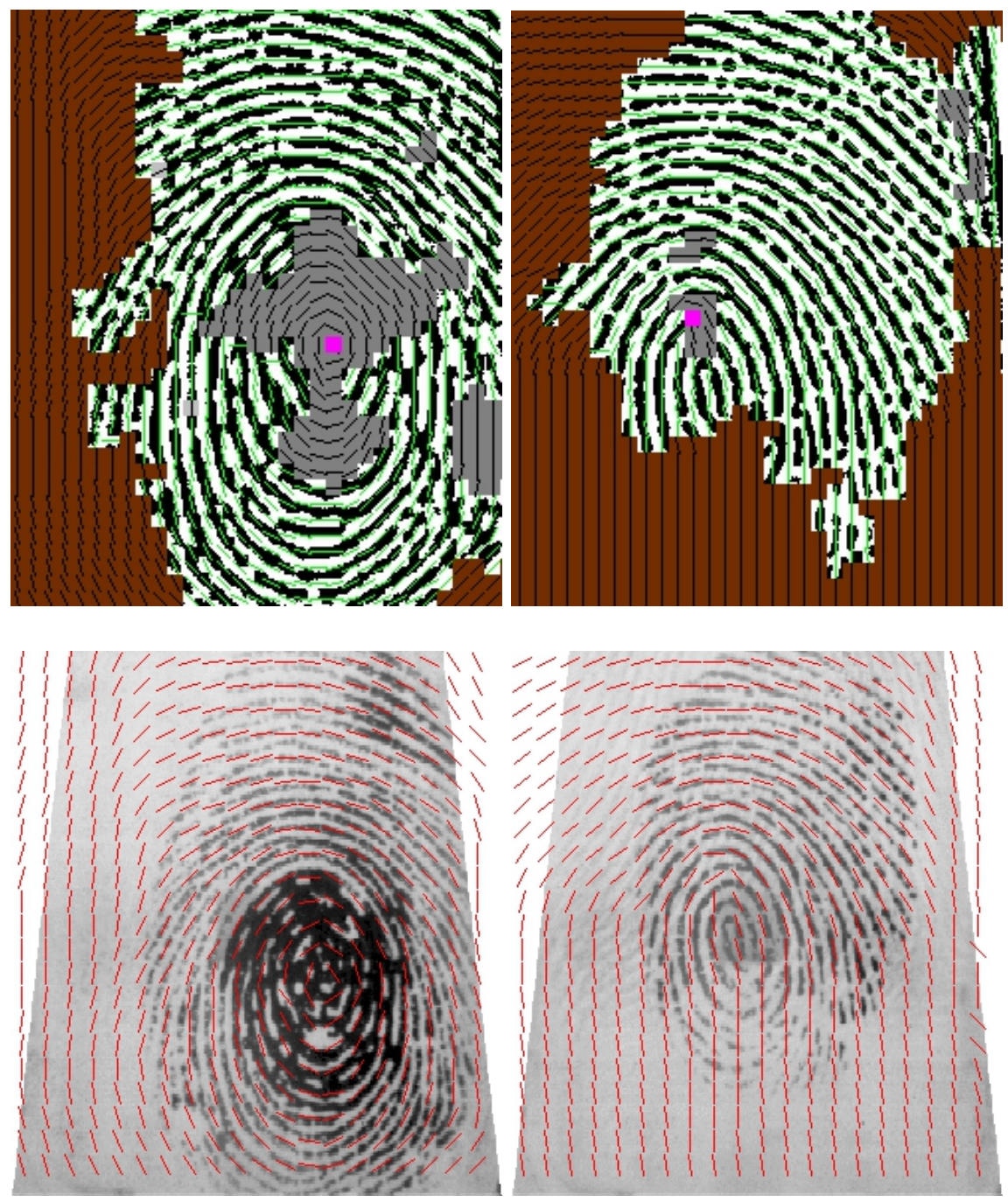

Figure 4.10: Two fingerprints of the class whorl. For the image on the left, the reconstruction of the orientation field leads to a satisfactory result. For the image on the right, it failed for two reasons: first, the location estimation of the upper core (block in magenta) is inexact, and second, the core below is missing. This is due to its location being in an area with too few parallel segments to be part of the coherent structure, and the singular point detection of the presented method is limited to gaps which are surrounded by blocks endowed with an orientation estimation.

portion of line segments originating from the tracing of noisy parts can be classified as 'recoverable'. If an area is not covered by the coherent structure, but it differs clearly from the background e.g. pertaining to the mean and variance of its gray values [9], then this quality corresponds to category 'unrecoverable'.

The OF estimation method described in Section 4.4.2 can effortlessly extrapo- 
late into 'unrecoverable' and background regions, but in terms of avoiding the extraction of spurious features and reducing computational costs for subsequent image enhancement, it is advisable to exclude these regions [3].

\subsubsection{Ridge Frequency Estimation}

Applying the merged coherent line structure and the orientation field, an estimate of the ridge frequency can be derived. This may be very useful, e.g. if a Gabor filter shall be applied in a subsequent image enhancement step (see Chapter 7).

\subsection{Experimental Results}

\subsubsection{Introduction}

In this section, it is the aim to analyze the robustness to noise of the line-sensor based method for OF estimation and compare its performance with the gradient based method [10] and the multiscale directional operator [119]. In a first step, a small number of good quality images was chosen in which foreground and orientations were manually marked. Next, artificial noise was added to these images mimicking scars and broken ridge lines, and the performance of all three methods was measured for various levels of noise. In the main part of the experimentation, 60 naturally poor images were chosen which represent the typical types of noise. In these images, 1782 orientations were manually marked with a focus on regions affected by noise. As described in Section 4.1, an estimated orientation that deviates considerably from the true ridge flow passed on to a Gabor filter tends to lead to the creation of spurious minutiae. According to a reviewers suggestion, hence, I will use the number of estimated orientations that deviate more than $15^{\circ}$ from the marked orientation as a measure for comparing the performance.

\subsubsection{Scar Simulation}

For our simulation, 15 good quality gray scale images were chosen from the FVC2000 [99] and FVC2002 databases [100]. The images were divided into blocks of $16 \times 16$ pixels. A human expert classified the blocks into foreground or background and determined the orientation for each foreground block. This 'ground truth' orientation will be used as the reference value for the performance evaluation. Scars are simulated by drawing white bars onto the original images. See Figure 4.7 for an example with 15 artificial scars. This type of noise simulation has the advantage that, like in the case of generating synthetic images [20,104], the level of artificial noise can be steered and a big number of images can be produced. Simulation runs were conducted for 5 to 25 scars in steps of 5 additional scars (see Figure 4.11). For each number of scars and each original image, 100 scar images were produced. The following parameters were randomly chosen (independent and identically distributed) in the given range:

- length (10 to 50 pixels and 10 to 200 pixels; see Figure 4.11)

- width (2 to 5 pixels) 

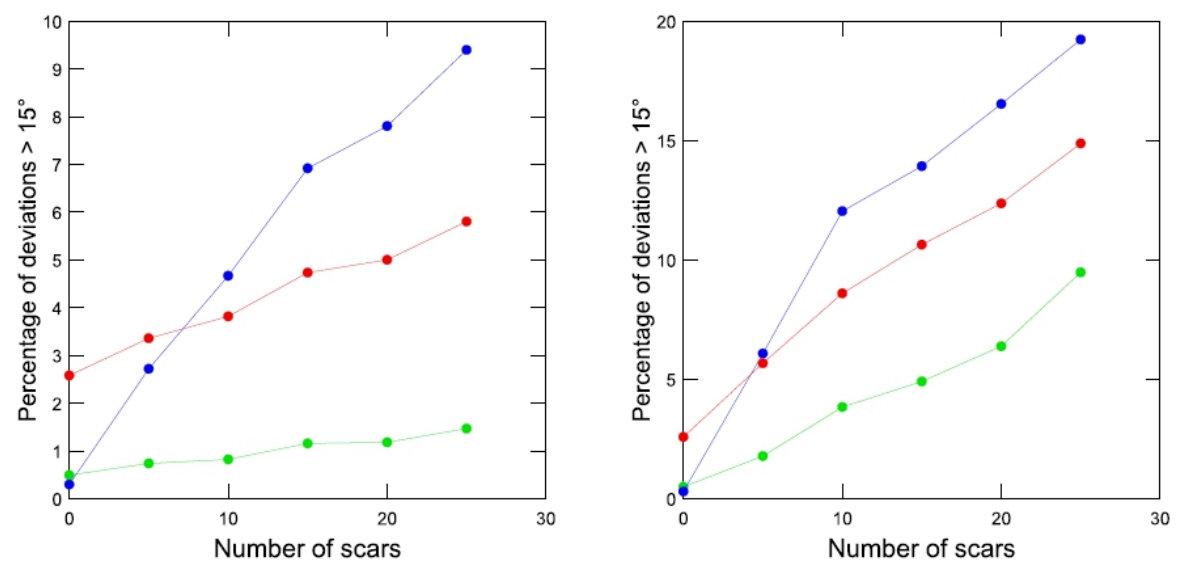

Line-sensor based orientation estimation

Gradients based orientation estimation

Multiscale directional operator

Figure 4.11: Performance comparison for different levels of artificial scar noise. The scar length was randomly chosen (i.i.d.) between 10 and 50 pixels (left) and between 10 and 200 pixels (right).

- orientation (0 to $\pi)$

- $\mathrm{x}$ - and $\mathrm{y}$-coordinate of the scar center (among all foreground pixels)

For the original images, the gradients based method performed slightly better than the line-sensor based method (12 vs. 20 estimations with deviation $>15^{\circ}$ out of 4072 foreground blocks). Most of those erroneous estimations by the line-sensor were caused by the difficulty of finding parallel line pieces in the core area. With an increasing number of simulated scars, the gradients based orientation estimation deteriorates proportionally to the amount of added noise. The performance of the multiscale directional operator is more stable than the gradients based method, but it is significantly worse than the line-sensor based method.

\subsubsection{Simulation of Broken Ridges}

In a simulation of broken ridge lines, I strove to mimic the type of noise caused by a dry finger. In my opinion, the artificial noise does not look as realistic as real noise, but nevertheless this procedures was very useful for testing the behavior of the three OF estimation methods. The line sensor method and the results of the noise simulations were presented at the 6th International Symposium on Image and Signal Processing and Analysis (ISPA) in Salzburg, Austria, held September 16-18 2009.

The results (see [47]) are similar to the previous experimentations with simulated scars. The OF estimation derived from the coherent structure of parallel 


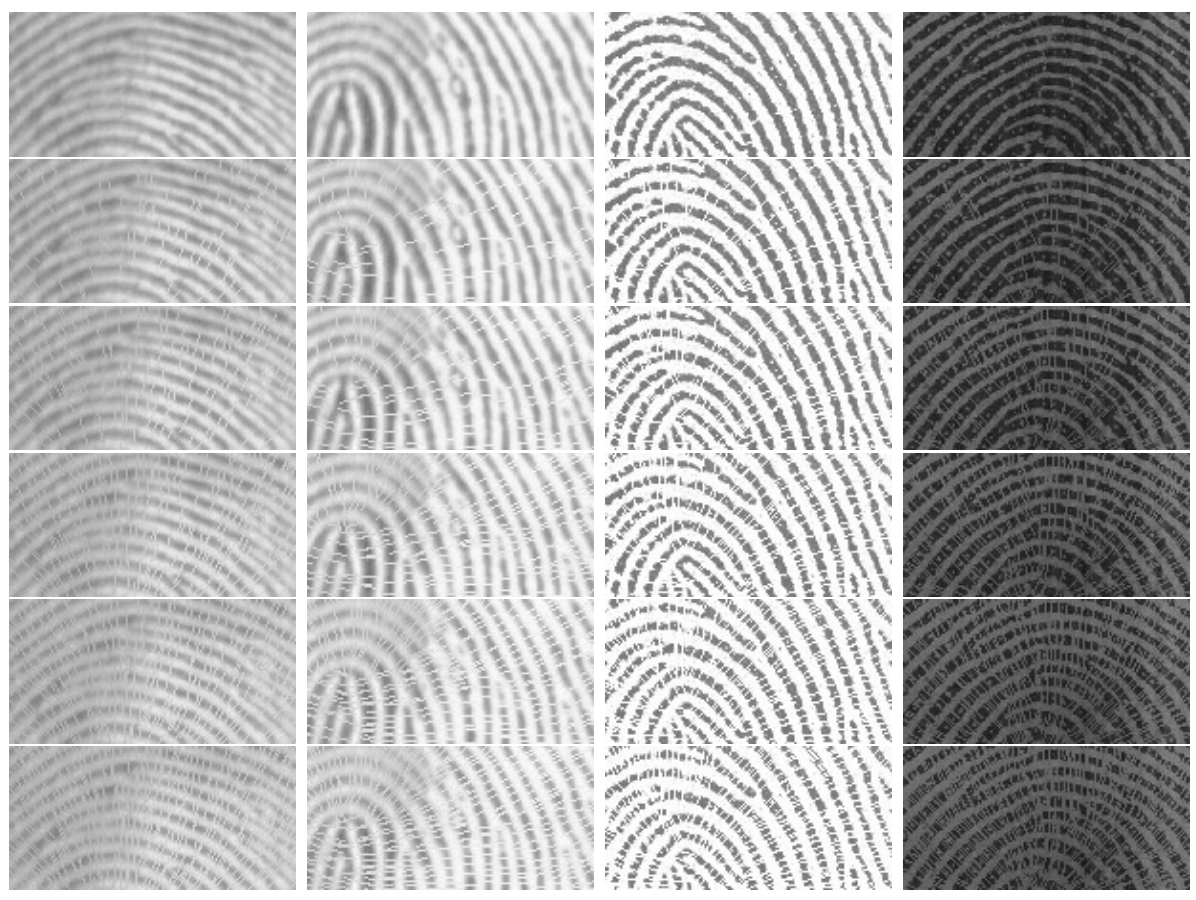

Figure 4.12: The top row depicts details from four images of FVC2000 [99] and FVC2002 [100]. Row 2 to 6 display simulations of noise levels from 500 to 2500 .

lines is less susceptible to this kind of simulated noise than the gradients based OF estimation and the multiscale directional operator.

\subsubsection{Real Life Noise}

For the benchmark test with real life noise, 60 poor quality images were chosen from FVC2004 [101]. This selection represents three typical types of noise (see Figure 4.2):

- Wet finger

- Dry finger

- Finger with scars

1782 orientations were manually marked with a focus on regions affected by noise. Selection criteria for the marked orientations were that ridge flow can be determined clear without ambiguity by a human expert and the location presumably poses a challenge to an automatic orientation estimation. All marked orientations and estimations by the line-sensor method consisting of the tuple image filename, $\mathrm{x}$ - and $\mathrm{y}$-coordinate and angle are made available for comparative tests. 


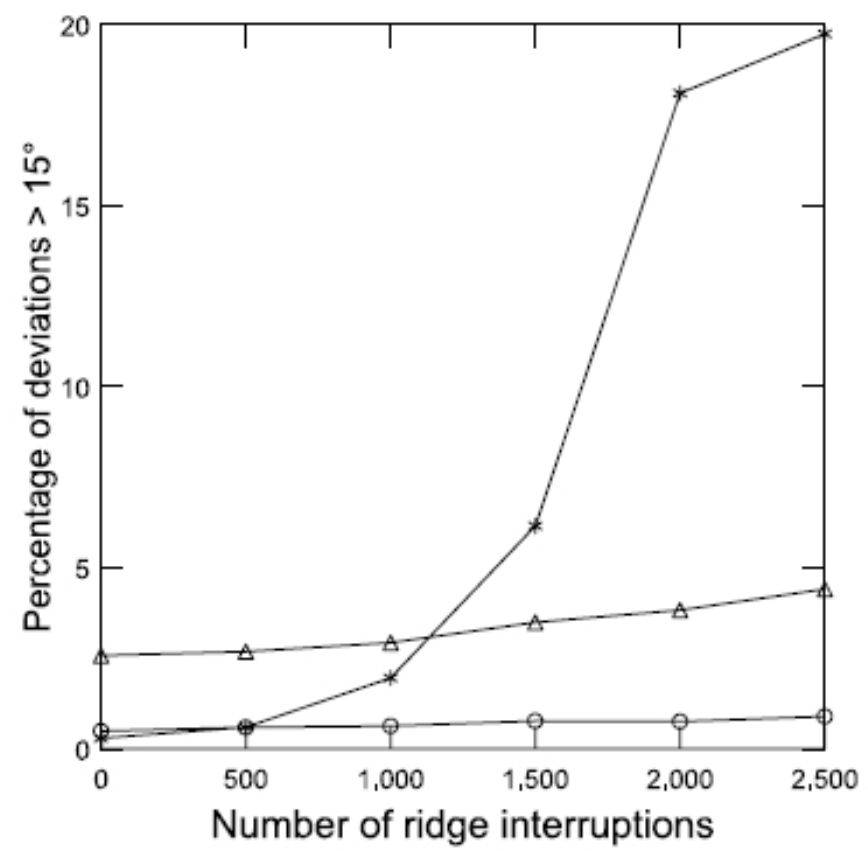

Line-sensor based orientation estimation

* Gradients based orientation estimation

$\triangle$ Multiscale directional operator

Figure 4.13: Performance comparison for different levels of simulated noise.

On these naturally poor fingerprint images (see Figure 4.14 for three examples), the line-sensor based method is compared with the gradients based method and the multiscale directional operator using the same performance measure as before. For the gradients based method, orientations were computed using averaging windows with sizes ranging from 5 to 101 pixels. Odd window sizes were applied, so that the marked location $(i, j)$ is the true center of the averaging window. If the window protrudes the image border, only the available gradients were accounted for. Results depending on the size of the averaging window are shown in Figure 4.15.

The multiscale directional operator [119] was calculated using the standard deviation as information parameter and with pixel-wise filtering as suggested by the authors. Orientation estimations were computed for 36 different combinations of the three parameters number of directions $D$, number of test points $n$ and the size of the smoothing window $\Omega$. For the number of directions, values in the range from 4 to 120 were tested.

As shown in Table 4.1, the line-sensor based orientation estimation deviates in 

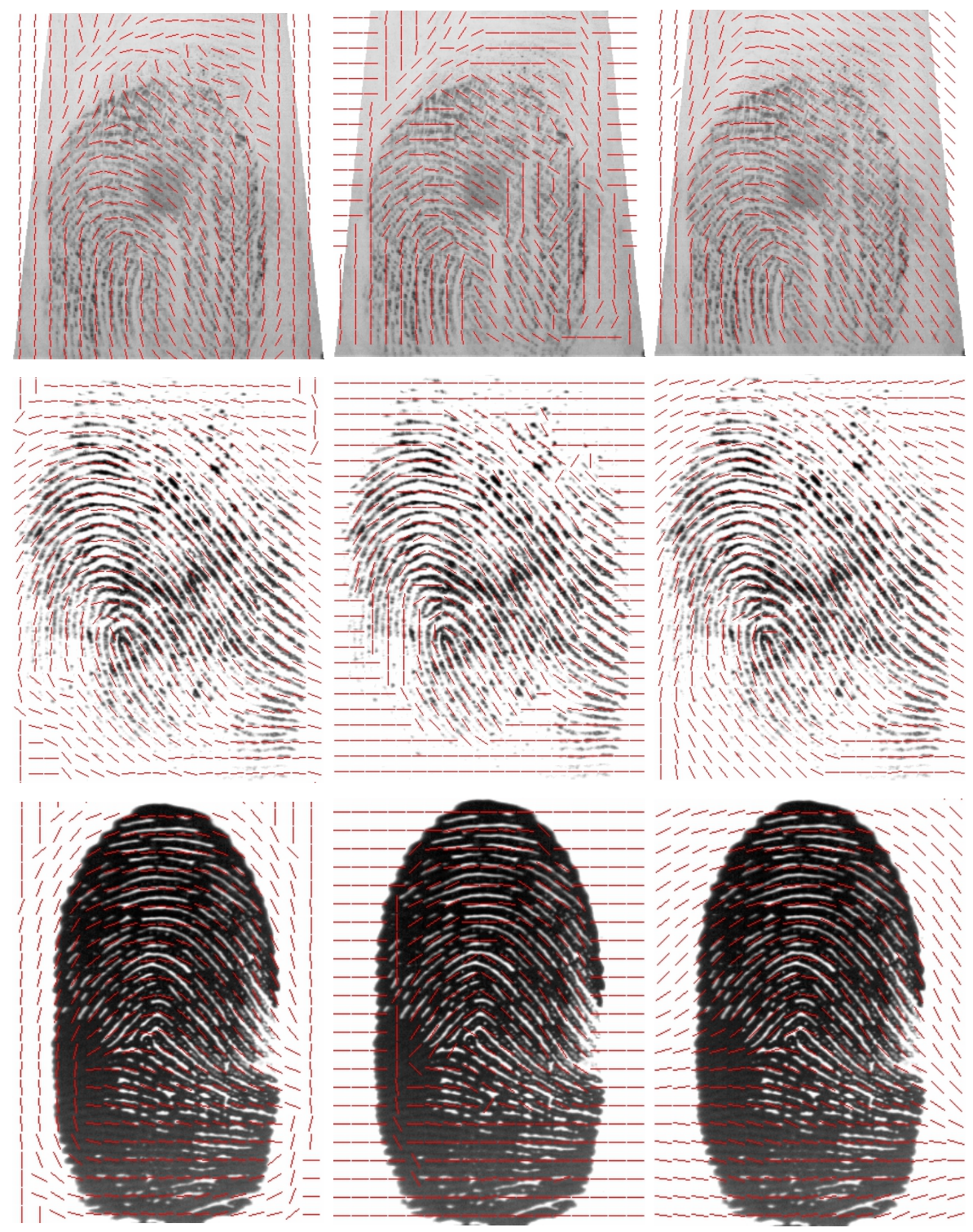

Figure 4.14: OF estimations for three images from FVC 2004 (top row: image 107_3 from database 2; below images $106 \_7$ and $101 \_8$ from database 1 ). The left column displays the gradients based estimation (with $W=59$ ), the central column the estimation of the multiscale directional operator (with $D=30$, $n=16$ and $\Omega=45$ ) [119] and the right column depicts the result of the linesensor based method. For illustrative purposes, the orientation at every 16th pixel horizontally and vertically is shown for the gradients based method and the multiscale directional operator which are endowed with pixel wise calculated orientation fields.

only 79 out of 1782 cases more than $15^{\circ}$ from the manually marked orientation, whereas the best result of the gradient based method was 119 erroneous esti- 

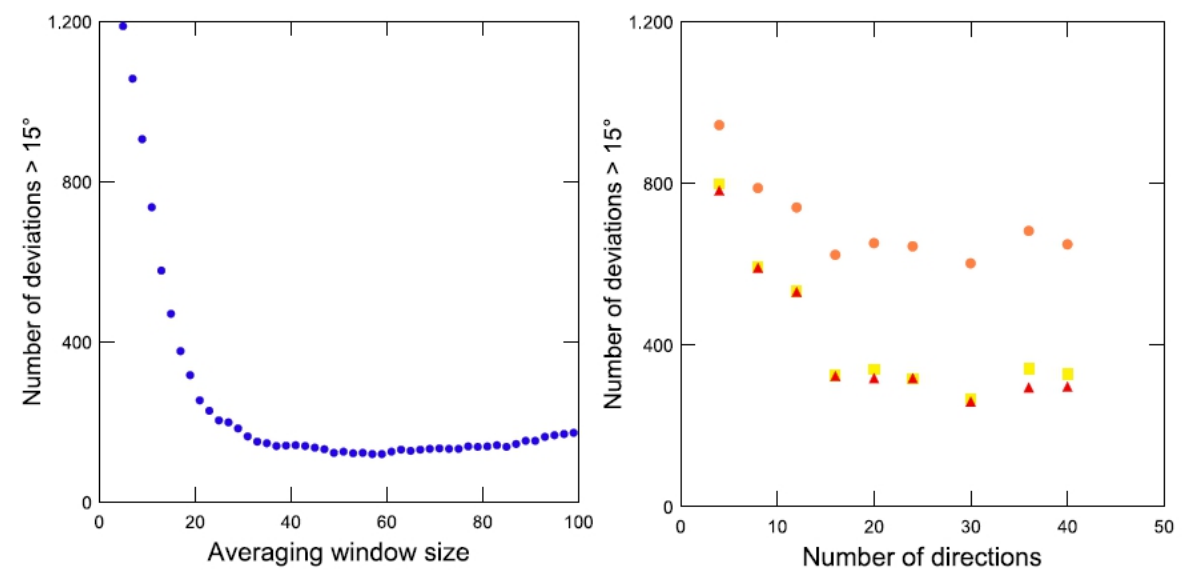

Figure 4.15: The performance of the gradients based OF estimation (left) and the multiscale directional operator (right) on 1782 marked orientations from 60 images of FVC2004. On the right, an orange circle indicates a smoothing window $\Omega$ of 15 pixels, a yellow square 31 pixels and a red triangle 45 pixels, respectively.

Table 4.1: Comparison of Orientation Estimation Performance on 1782 Marked Orientations from 60 images of FVC2004 [101]

\begin{tabular}{|c|c|}
\hline Orientation Estimation Method & Number of deviations $>15^{\circ}$ \\
\hline Line-sensor & 79 \\
\hline $\begin{array}{l}\text { Gradients } \\
\text { Best results with } \\
w=57 \text { and } w=59\end{array}$ & 119 \\
\hline $\begin{array}{l}\text { Multiscale directional operator } \\
\text { Best result with } \\
D=30, n=16 \text { and } \Omega=45\end{array}$ & 259 \\
\hline
\end{tabular}

mations for averaging window sizes of 57 and 59 pixels. The best performance of the multiscale directional operator was 259 deviations of more than $15^{\circ}$. 
This result demonstrates the robustness and effectiveness of the line-sensor based approach for orientation estimation in regions of fingerprint images which are disturbed by real life noise.

\subsection{Discussion}

\subsubsection{Application Areas and Computational Complexity}

In comparison to the gradients based method, the presented line-sensor based method can estimate the flow of ridges and valleys for noisy fingerprint image regions in a significantly more robust way as shown in Section 4.5.4. On the other hand, it has the disadvantage of a greater computational complexity than the gradients based approach. Hence, the choice of the OF estimation method will depend on the area of application. In forensics, the benefits of a more reliable orientation estimation can outweigh computational costs, whereas in on-line fingerprint verification fast computation is of great importance.

Bearing in mind these two different areas of application, the further development of the line-sensor based method for OF estimation should go in two directions: one is the formulation of a 'light version' that follows the principles of the line-sensor and the coherent structure of parallel segments and meets the computational requirements and time constraints of on-line verification systems. The second direction is the improvement of the 'forensic version' with aim of obtaining all the ridge flow information that is present in a fingerprint image, and simultaneously providing a segmentation into foreground and background as well as a local image quality estimation which can be used for assessing the reliability of extracted features at the matching stage. In this way, a subsequent image enhancement step is optimally prepared.

The 'light version' will be presented in the next section and the 'forensic version' is sketched in Chapter 5 and some first experimental results are presented. In addition, it will be of interest to apply the presented method to palmprints and fingerprint latents (and compare it e.g. to [153]).

\subsection{Fast Line Sensor}

In this section, the line sensor method described in Section 4.3 using straight lines is further developed into the direction of a 'light version' as announced in [46]. The general idea is to improve the method described in Section 4.3 by overcoming the drawbacks of the line tracing while preserving its advantages. One major strength of the line sensor is the robustness to noise, as discussed in Section 4.6 two weaknesses are the computational complexity and the limited accuracy in the core zone. Both drawbacks are connected to the tracing process, whereas, in my opinion, the robustness of the method is provided by the coherent structure.

The major differences between the 'light version' and the line sensor from Section 4.3 are: 

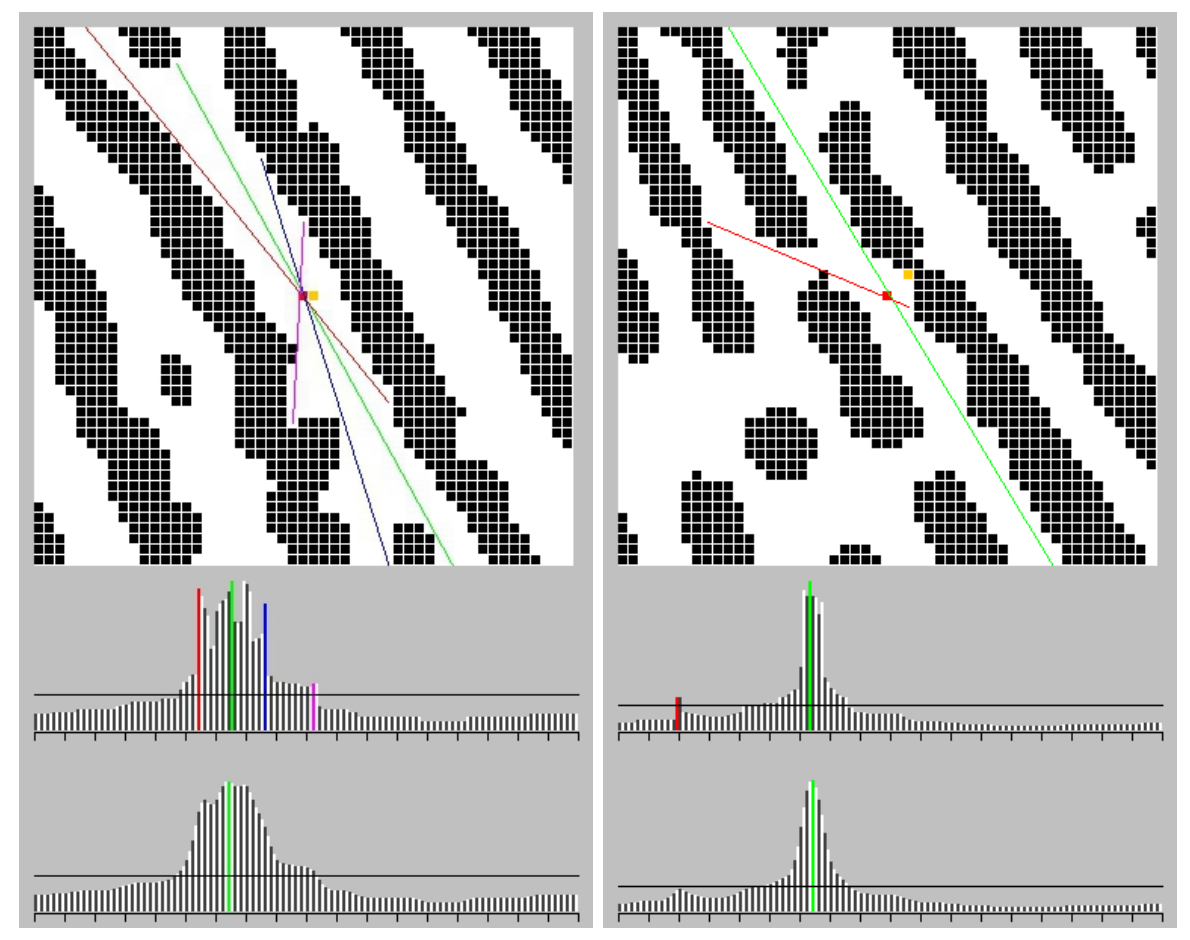

Figure 4.16: First, the grid points (orange square) are centralized, and from these start points (red square), the line sensor peeks into directions all-around. The length of opposite directions is summed up for the orientation histogram, depicted below. In the image on the left, the lines corresponding to the four local maxima are shown. Next, the orientation histograms are smoothed with a Gaussian kernel. For these two examples, the resulting histograms have only one maximum. Please note that local maxima in the neighborhood of a greater local maximum are ignored (here: minimum distance min $_{\text {dist }}=10$ ). The black horizontal line on top of the histograms indicates the minimum line length (here min $_{l e n}=15$ pixels). 
- no tracing is performed

- at each grid point, multiple directions can be considered

- all steps of the method are fully computable in parallel

In the subsequent paragraphs, all details of the implementation are described with an emphasis on the parts that differ from the previous version of the line sensor.

\subsubsection{Preprocessing}

The first step of the method is the preprocessing of the gray scale input image. This is performed exactly as described in Section 4.4.1 and results in a binary image which is stored as two dimensional Boolean array.

\subsubsection{Line Sensing}

Next, we iterate in grid with size $g s=8$ pixels over the binary image. At each grid point, the sensor start point is first centralized and then straight lines are sensed by Boolean comparison with precalculated lines as described in Section 4.4.1.

Centralization Purpose of the centralization is to avoid start points in 'pockets' of the coarse binary image. For a grid point, the 8-connected neighbors of the same color are determined iteratively in the binary image up to a maximum number of neighbors. Then, the barycenter of all neighboring pixels is computed and the nearest pixel of the same color as the grid pixels is chosen as start point. This procedure has an effect like inflating a balloon and the barycenter tends to move towards the center of the ridge or valley with each iteration (see Figure 4.16 , image on the right for an example).

Straight Line Sensing For each start point, the lines are sensed in the binary image by Boolean comparison of possible pixel steps using precalculated lines. In our experimentation, lines for $n=360$ directions are computed during the initialization of the line sensor.

At this point, the previous version of the line sensor chose the best direction (i.e. the direction with the maximum number of possible pixel steps without changing the color) in a all-around search, or while following a line, for a defined cone. Here, the length of opposite directions are summed up into an orientation histogram (see Figure 4.16). Next, the histogram is smoothed with a Gaussian filter which bundles the main directions and erase small peaks caused by noise (see Figure 4.16, the sensor on the right). However, larger scars that cross two or ridges are not removed by the Gaussian smoothing. They will be stored in a list together with all other detected segments and the decision which segments reflect the true ridge flow will be postponed. The alteration of permitting more than one segment per sensor is also helpful for representing curved lines, especially close to the core point. 

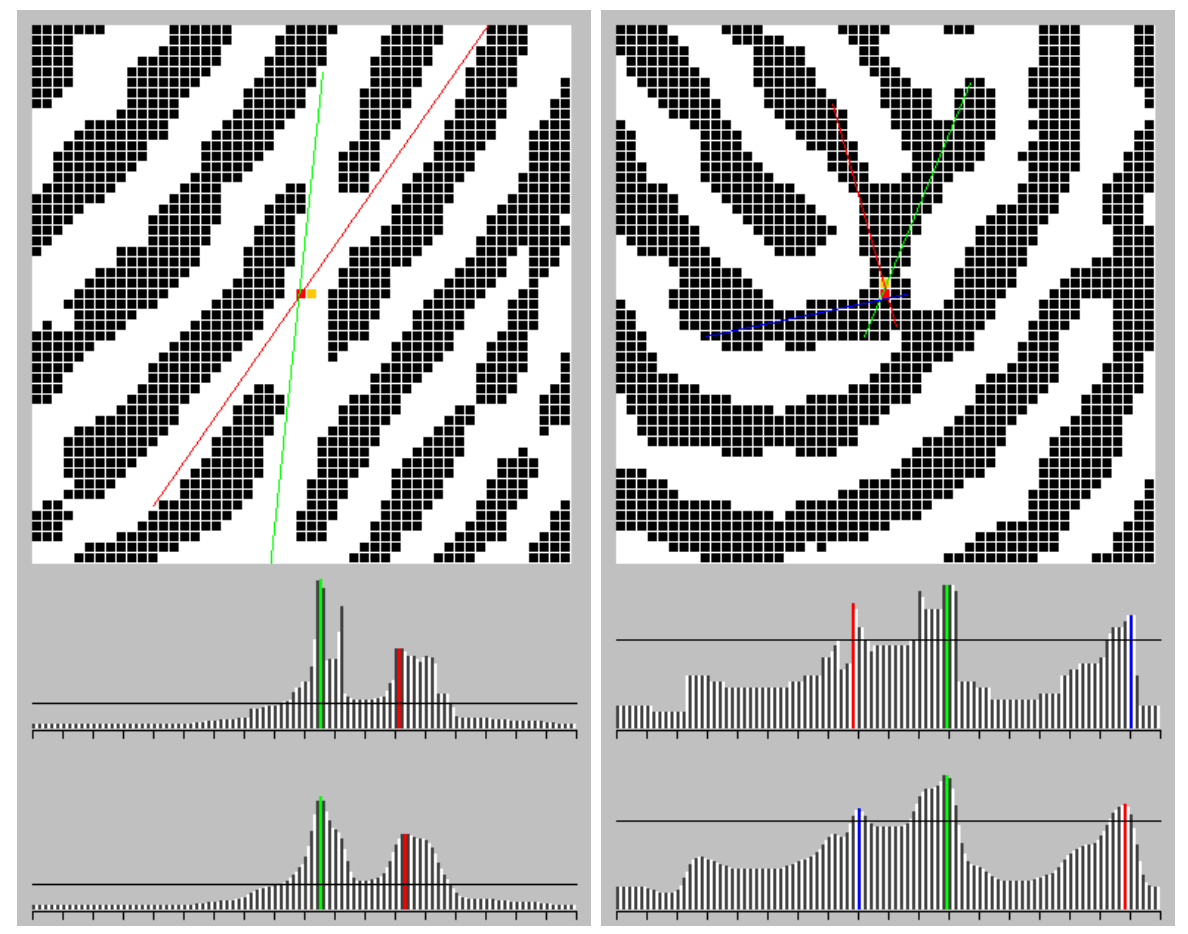

Figure 4.17: In the example on the left, the red line reflects the true ridge flow, whereas the green line belongs to scar. Both segments are stored for a later evaluation. During the growing of the coherent structure, the wheat will be separated from the chaff. The start point on the right is closely located to the core and all three lines are valid. Whether they have an impact on the OF estimation depends on the discovery of parallel segments. 


\subsubsection{Coherent Structure}

The growing coherent structure is build as described in Section 4.4.2 with one important difference: previously, it was time consuming to discover the neighbors for each line segment. Here, this piece of information is encoded into the grid index which saves a lot of time. Also, the tuning parameters of the growing process have to be adjusted with regard to fact that much more segments are available. A variety of constants was chosen empirically in order to optimize the performance of the orientation field estimation. All values are listed at the end of the section.

\subsubsection{Orientation Field Estimation}

All line sensors which are part of the merged coherent structure broadcast their orientation as explained in Section 4.4.2. Two examples are given in Figure 4.18 in which the merged coherent structure is depicted in the left column and the derived orientation field in the right column.

An alternative approach for this step is also thinkable: several authors proposed methods for the reconstruction of fingerprints from minutiae files (see e.g. [132] or [21]). Triplets of good quality minutiae form a triangle and the orientation inside the triangle is interpolated using the orientation of the three minutia points. A very similar idea is applied in [19] for speeding up the manual marking process of a fingerprint by a human expert. For reasonable number of points, the orientation and ridge frequency is marked and the OF and RF map is interpolated using Delaunay triangulation [31] (see Figure 7.1 for illustration), a technique well known from minutiae matching [93]. Analogous, the orientation can be estimated a sufficient number of points from line sensors and afterwards, the OF is interpolated using Delaunay triangulation. However, according to my experience it is difficult to ensure a good quality of the OF estimation near the core points applying this method.

\subsubsection{Parameters}

If we refer to a line sensor based OF estimation in the subsequent chapters, it was obtained with the following parameters:

- number of directions $n_{d}=360$

- number of orientations $n_{o}=180$

- $\operatorname{grid}$ size $g s=8$ pixels

- minimum line length $\min _{l e n}=15$ pixels

- maximum number of discovered lines exceeding the minimum line length $\max _{\text {lines }}=60$

- maximum number of local maxima in the orientation histogram $\max _{\max }=3$

- maximum angle for segments to be classified as parallel $t h r_{\text {parallel }}=8^{\circ}$ 

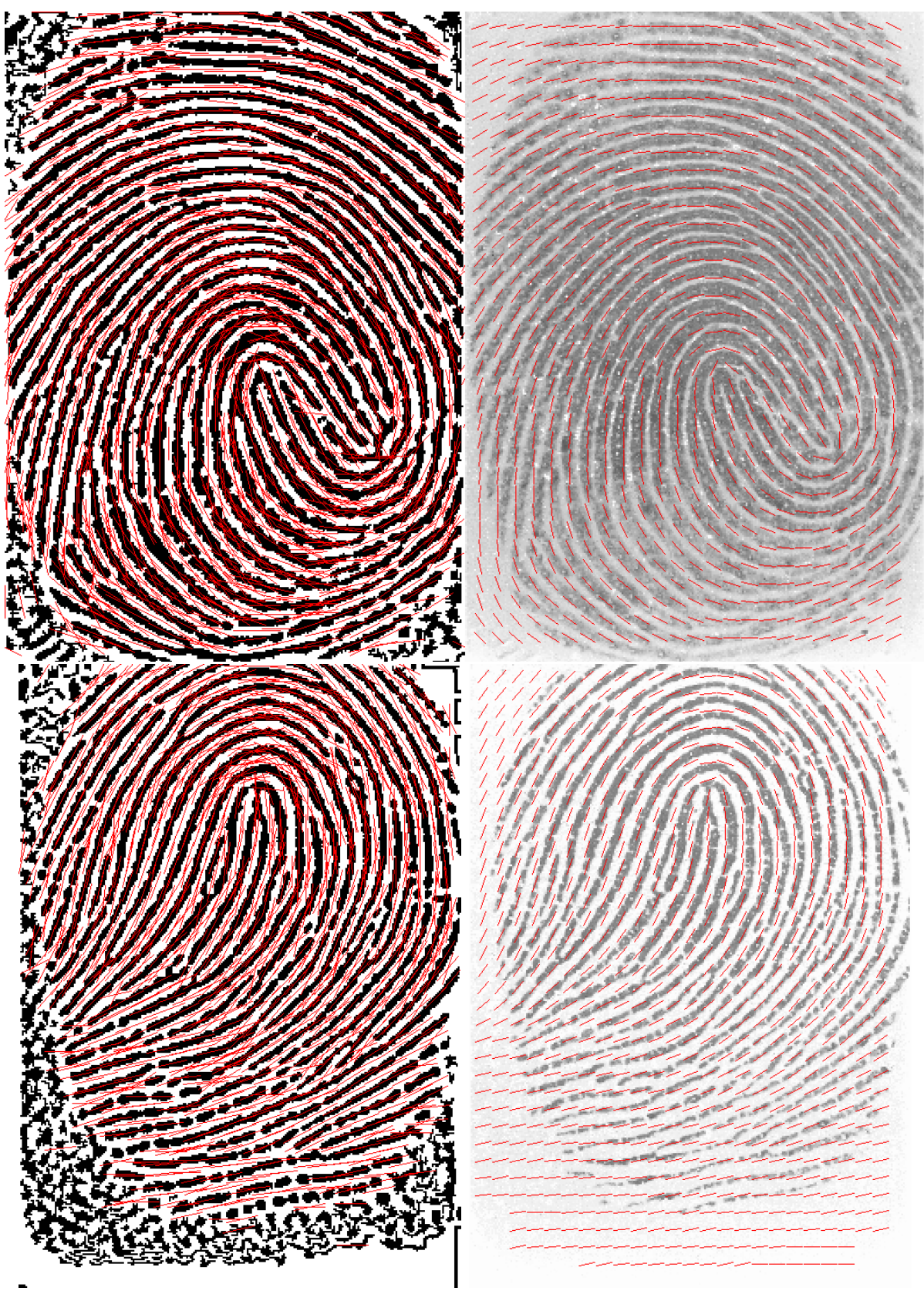

Figure 4.18: Left column: line sensors which are part of the merged coherent structure plotted on top of the binary image. Right column: derived OF estimation on top of the original image. 
- minimum number of parallel segments for a seed segment min $_{\text {seed }}=25$

- search radius (in grid index distance) $s r=3$

- minimum number of parallel segments for entering a coherent structure min $_{\text {enter }}=5$

- minimum structure size for merging $\min _{c s}=100$ segments

\subsubsection{Discussion}

In comparison to the previous version of the line sensor (see Section 4.3), the waiving of the tracing did not only speed up the OF estimation, but also increased the accuracy in the core region (see Figure 4.18 and Figure 4.19). The latter is facilitated by allowing more than one orientation for each sensor. E.g. at locations close the core, the sensor can detect two lines which form an angle $>15^{\circ}$. If both are valid reflections of the ridge flow and they have enough parallel lines (with respect to a threshold), then including both lines into the OF estimation tends to increase the precision of the estimation. Previously, this was impossible because of the way the tracing is designed (see Figure 4.20 for a comparison of both methods).

In addition, all steps of the line sensor light version can be computed in parallel. For the results in Chapter 7 and 8, a proof-of-concept implementation programmed Java ${ }^{2}$ was applied. In order to demonstrate the practicability of the line sensor based OF estimation for on-line fingerprint verification, it is desirable to implement the algorithm using the parallel processing capabilities of graphics processing units (GPUs) and measure the average runtime for fingerprint images from the FVC databases and other sources obtained with different scanners.

${ }^{2}$ http://java.sun.com/ 

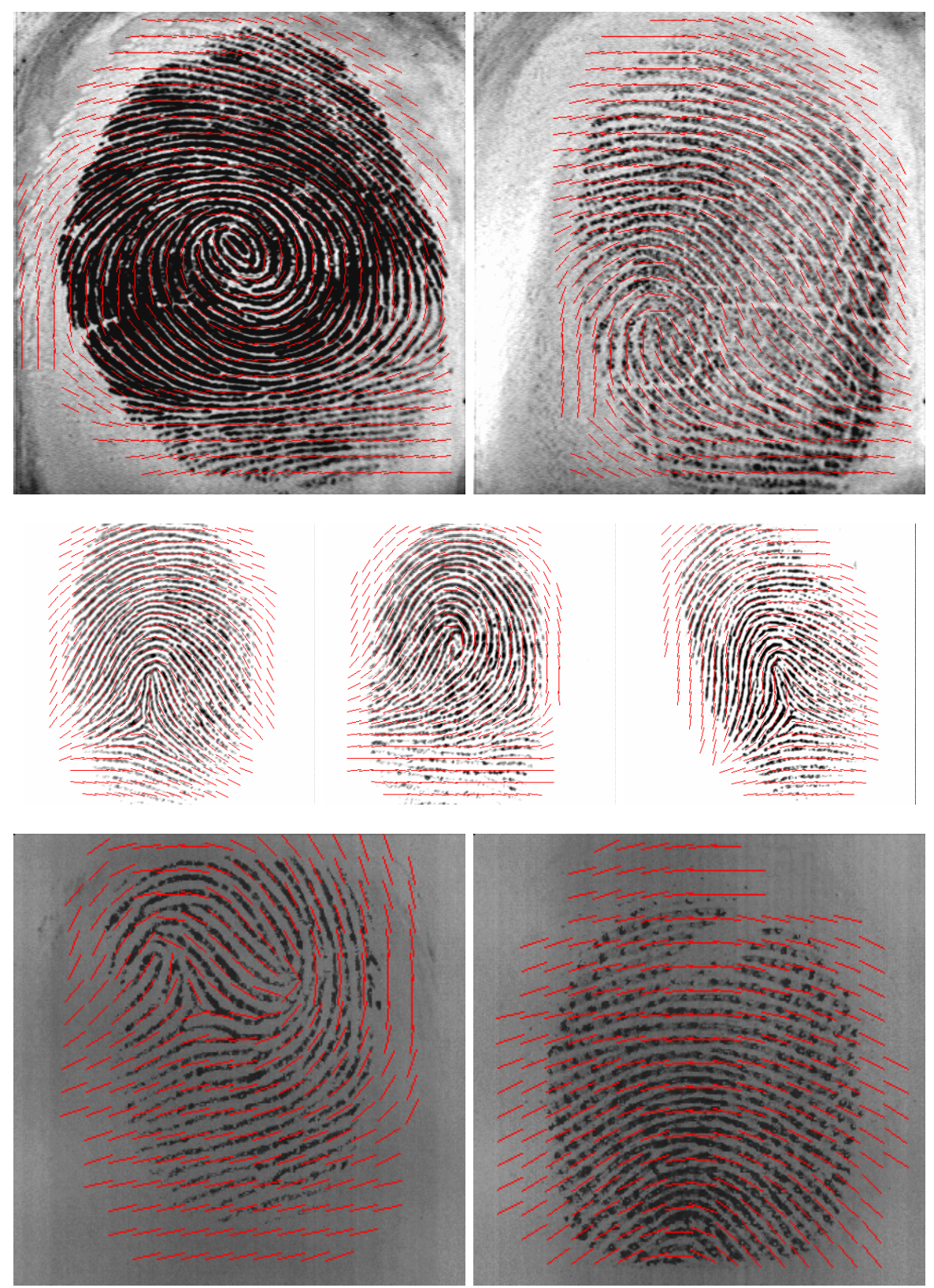

Figure 4.19: Line sensor OF estimations for a selection of images which illustrate the capability of the 'light version' to adapt to core regions with a precision that was not possible in the previous version due to the tracing procedure. 

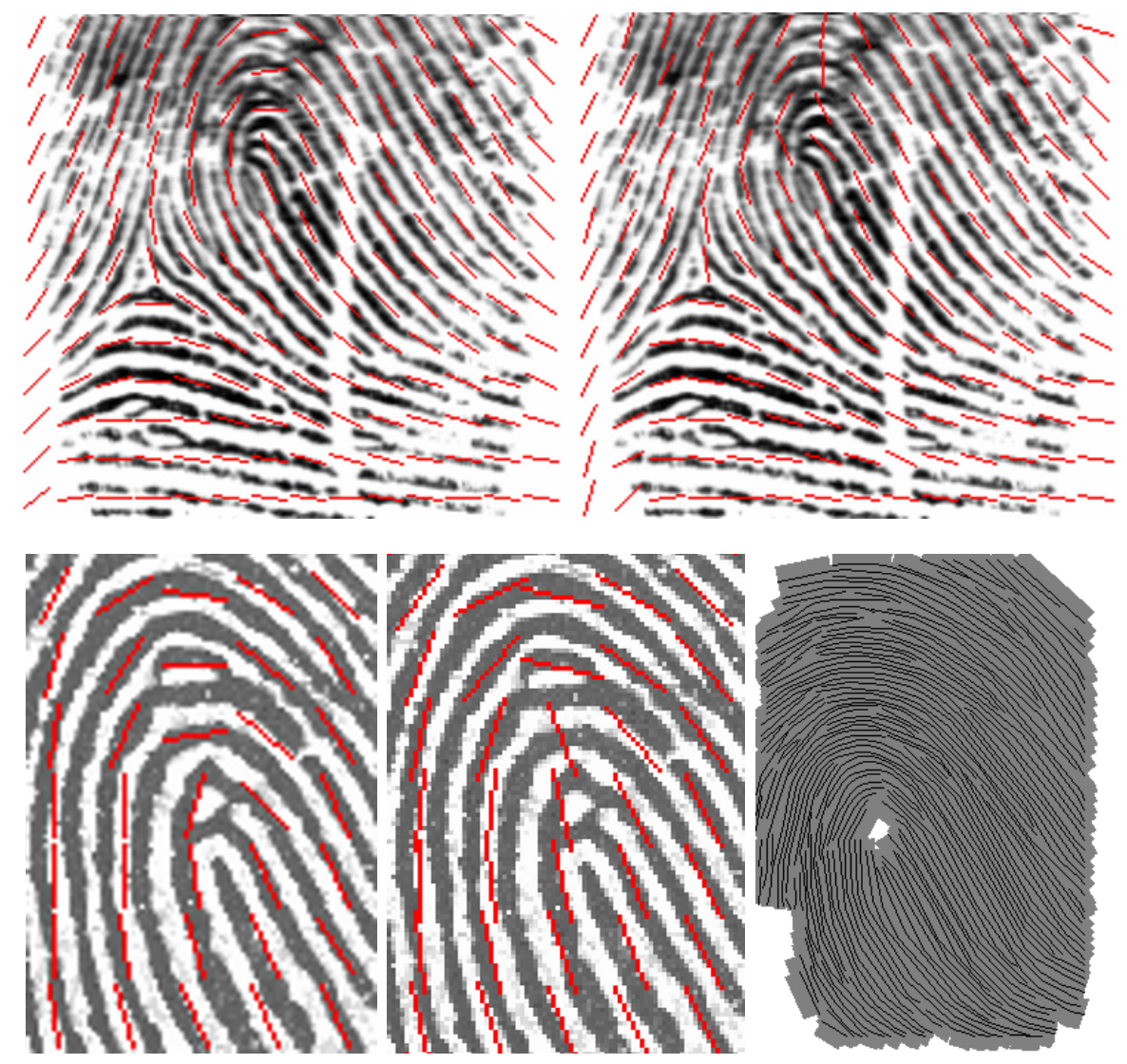

Figure 4.20: Details from images 2_1 of FVC 2004 database 1 (top row) and $30 \_6$ of FVC 2000 database 2. Comparing the fast line sensor based OF estimation (left column) with line sensor method tracing ridges and valleys (second from left). The latter method traces the core area of some images only partially due to the cone criteria of the ridge tracing (see Section 4.4.1) or if it is traced correctly, the segments may lack enough parallel segments in order to become part of the coherent structure (bottom right). In the majority of the cases, the reconstruction described in Section 4.4.2 resolves the situation, but if the core location estimation is too imprecise, the result of the reconstruction is an erroneous OF estimation for the core region. The example on the top right illustrates the strengths and weaknesses of the method. The orientation estimation is accurate for the area disturbed by the scar and the dryness of finger, but it is wrong for the region above the core which is located close to the image border and it is incorrectly reconstructed. The fast line sensor method overcomes these hindrances by considering all possible orientations at each grid point. 


\section{Chapter 5}

\section{Line Sensing Based on Quadratic Bezier Splines}

The curved ridges and valleys of fingerprints resemble, at least in parts, quadratic Bezier curves. Therefore, it is a quite natural approach to endow the previously described line sensor which currently senses only straight lines with capabilities to sense quadratic Bezier curves [134]. This approach accounts especially for regions of high curvature like the core region in fingerprints of the types loop and whorl, but also for the central regions of arch type fingerprints. Quadratic Bezier curves are based on Bernstein polynomials and defined by:

$$
C(t)=\sum_{i=0}^{2}\left(\begin{array}{l}
2 \\
i
\end{array}\right) t^{i}(1-t)^{2-i} P_{i}=(1-t)^{2} P_{0}+2 t(1-t) P_{1}+t^{2} P_{2}
$$

where $P_{0}$ and $P_{2}$ are the start and end points of the curve and $P_{1}$ is the central control point.

Splines are functions defined piecewise by polynomials. Here, we combine two quadratic Bezier curves to a spline, analogously to the combination of two linear curves in the previous chapter. Very related are B-splines [134] for which the polynomials are defined by a different basis. To my knowledge, there are two publications that suggest the application of B-splines for fingerprint recognition. In [26], the usage of B-splines for the compression of fingerprint data is proposed. More recently, the removal of digitization errors in the binary, thinned image by B-splines was recommended [14].

Analogous to the precalculated straight lines, quadratic Bezier curves were computed for combinations of start points $P_{0}$ and end points $P_{2}$ of the control polygon with a Chebyshev distance $d$ in the range from 15 to 42 pixels. The curves were rasterized and stored in a tree [87] structure. Instead of checking hundreds of millions of curves, due to the tree structure the computational costs shrink down to a few tens of thousands Boolean comparisons in the binary image. See Figure 5.1 for an example of two discovered curves in a valley (white) and on a 


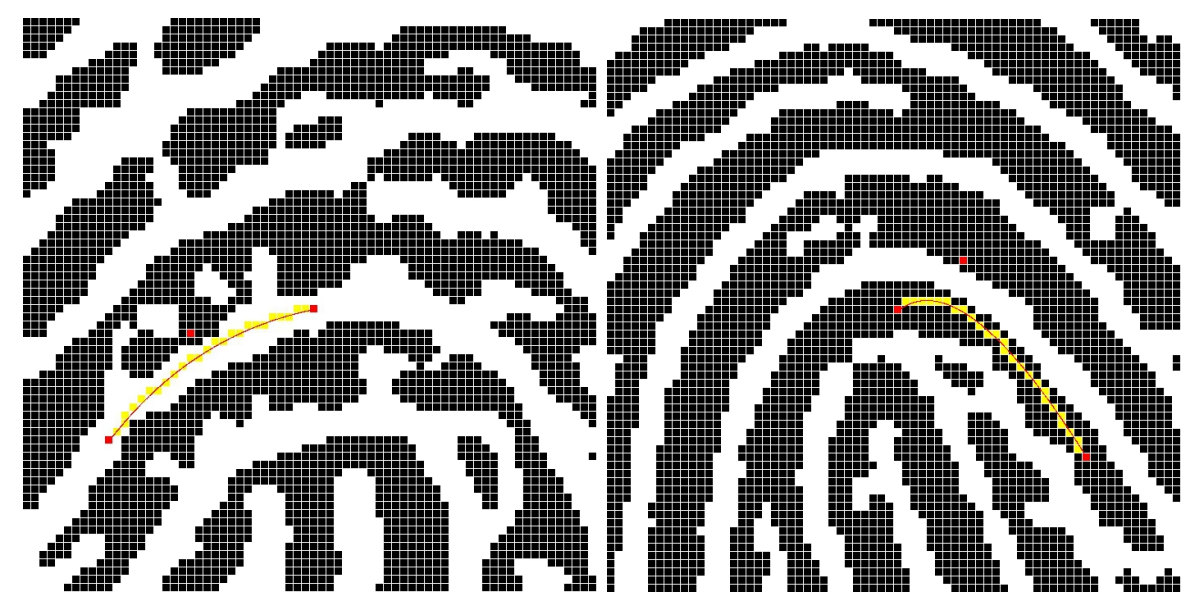

Figure 5.1: Peering around from the start point (red) in the center of the image, the sensor discovered a rasterized quadratic Bezier curve (yellow). The points of the control polygons are depicted in red.

ridge (black) close to the core point.

For combining two curves and preparing the creation of a coherent structure similar to the algorithm presented in Section 4.4.2, for each curve a score is calculated. The following weight factors where empirically determined on a selection of images which is representative for the typical types of noise:

- length of the curve $(80 \%)$

- degree of curvature (15\%)

- centralization of the end point (5\%)

The length of the curve is weighted positively and has a big influence. This contributes to the robustness of the method, because long segments are generally more trustworthy (with the exception of long scars which will be handled during the growing of the coherent structure). The curvature is weighted negatively, so that in the case of two approximately equally long segments, the straighter segment is preferred over the more curved one. And finally, among curves which are similar with respect to length and curvature, the one with more centered end point is chosen, because it is more likely to be connectible with further segments.

Any two quadratic Bezier curves can be combined, if the following the constraint is fulfilled: the angle formed by the central points of both control polygons and the sensor start point has to be close to $180^{\circ}$ (see Figure 5.2 for an illustration). This condition assures the continuity at the connection point. For all connectible pairs, the scores are summed up, and the combination with the highest score is chosen by the sensor. See Figure 5.2 for two examples of combinations with highest score for the respective sensor start point.

First results are very promising and it will be of interest to further investigate 

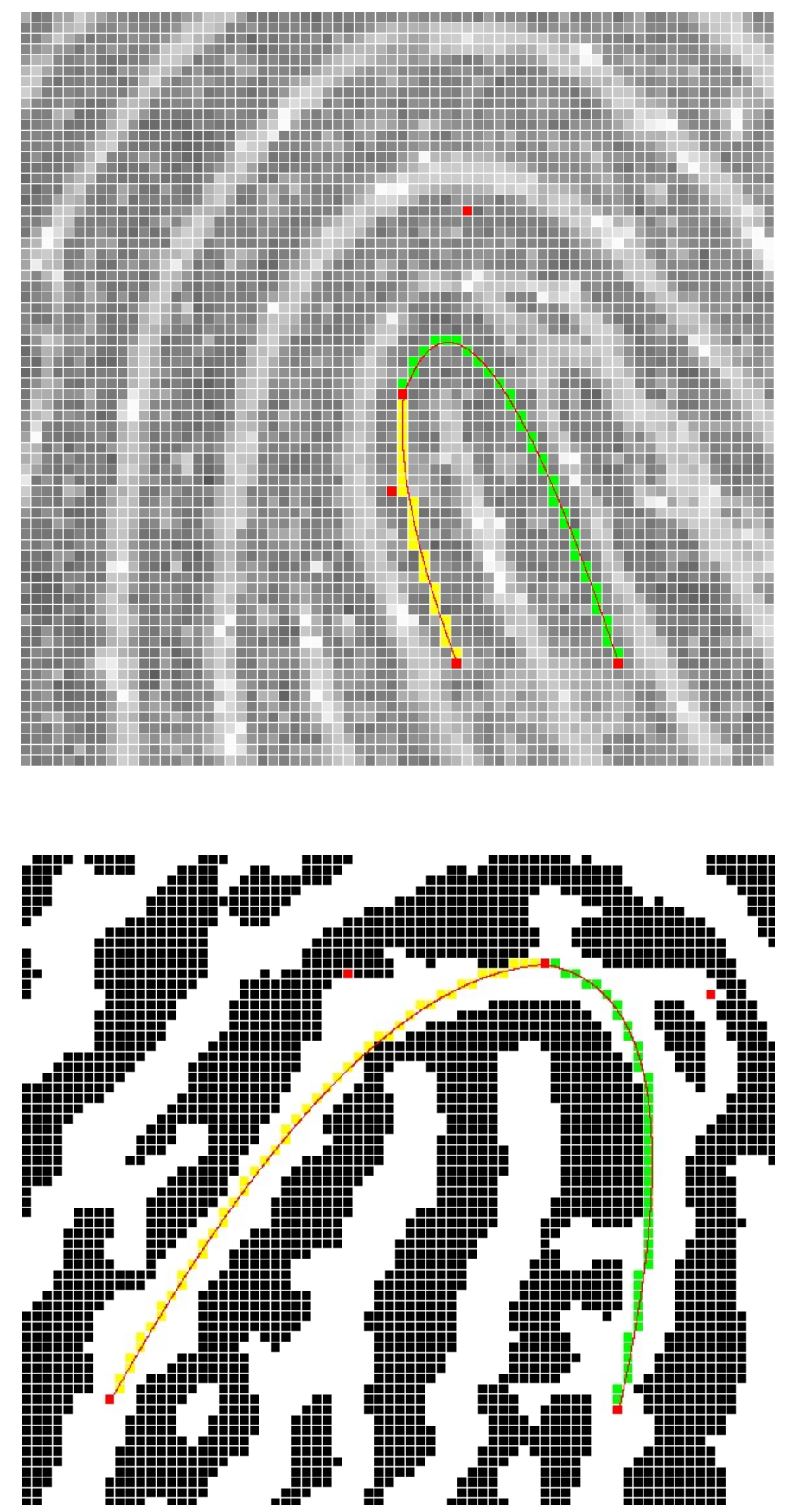

Figure 5.2: Two rasterized quadratic Bezier curves (green and yellow) and the points of the control polygons (red). The sensor start point connecting both curves is also the start point of both control polygons. The curves were detected in the binary image and, for image at the top plotted onto this detail of the grayscale image. For the image at the bottom, please note the centralization of the end points which increases the possibilities to connect additional curves. 
the application of quadratic Bezier curves to fingerprint images, particularly with regard to noisy core regions, for orientation field estimation, ridge frequency estimation and image enhancement. In the next chapter, this idea is adopted and curved regions are defined by a piecewise linear approximation of the curves. Chapter 7 introduces a curved Gabor filter which applies this idea to image enhancement. 


\section{Chapter 6}

\section{Ridge Frequency Estimation}

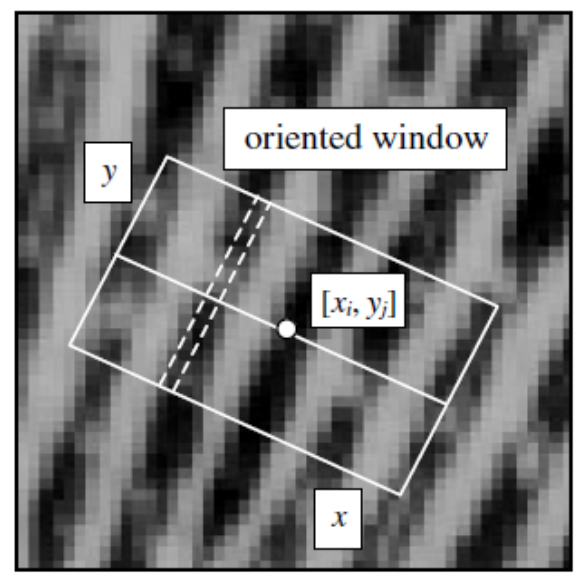

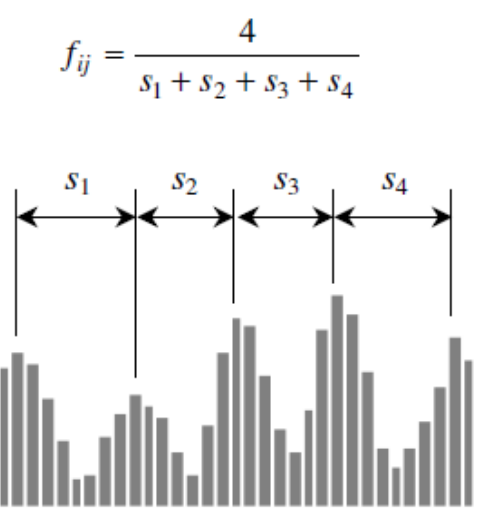

$x$-signature

Figure 6.1: Screenshot from [103], illustrating the ridge frequency estimation method called 'x-signature' proposed by Hong, Wan and Jain in [60].

In addition to the local ridge orientation, the ridge frequency $(\mathrm{RF})$ is a second local feature providing significant contextual information. It is defined as the inverse of the inter-ridge distance, measured orthogonal to the local orientation.

The ridge frequency is required by some image enhancement methods of which the Gabor filter is the most prominent one. It can vary considerably across different regions of a finger. If the ridge frequency is estimated incorrectly and these erroneous estimations are passed on to a Gabor filter, this can result in the creation of false additional ridges or the deletion of true ridges, depending on the tuning parameters of the filter, and possibly the number of iterations. An illustration of this effect is shown in Figure 6.2. Therefore, the quality of the ridge 

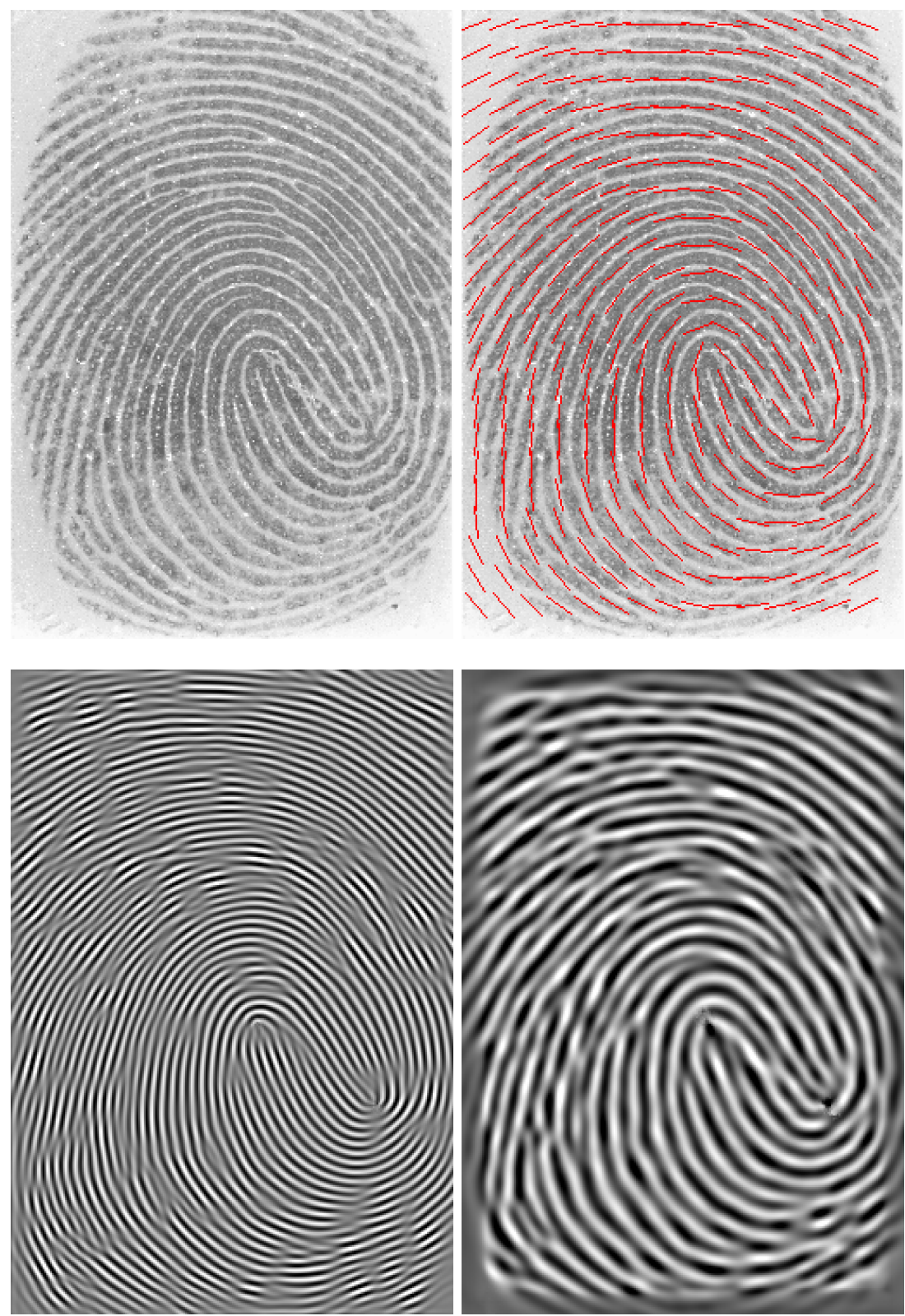

Figure 6.2: In the top row, the original image (left) 55_6 from FVC2000 database 2 and the line sensor based OF estimation (right) are displayed. The two images at the bottom were processed with a Gabor filter. The ridge frequency was globally set to $f=5.0$ for the image on the left and $f=15.0$ for the image on the right. The other filter parameters were identical for both images. 
frequency estimation can impact the overall performance, corresponding to the previous argumentation concerning the quality of the orientation estimation.

\subsection{Related Work}

\subsubsection{X-Signature}

A widely cited ridge frequency estimation method called 'x-signature' was proposed by Hong, Wan and Jain in [60]. The y-axis of a rectangular window centered in $\left(x_{i}, y_{i}\right)$ is aligned to the ridge orientation by rotation (see Figure 6.1). Gray-level values are averaged along the y-axis resulting in smoothed ridge and valley profile. The average distance between consecutive peaks in this profile is computed and the RF estimation is obtained by inverting the average distance.

\subsubsection{Higher Order Spectrum}

In order to achieve less error prone frequency estimations from a noise gray-level profile, [78] suggested the application of higher order spectrum which exploits the harmonics of the frequency. This approach is based on the assumption that the gray-level profile has in principal a sinusoidal shape which is disturbed by noise. A so called mix-spectrum is proposed for suppressing the noise.

\subsubsection{Geometric and Spectral Approach}

A two-step method for RF estimation is proposed in [86]. In the first step, a geometric method similar to the $\mathrm{x}$-signature and a spectral method based on harmonic coefficients are considered for estimating the ridge frequency of $64 \times 64$ pixels blocks. In the second step, the RF image is completed by the interpolation of missing values with diffusion equations.

\subsubsection{Short Time Fourier Transform}

The authors of [25] follow an approach similar to the x-signature and apply short time Fourier transform analysis for smoothing their RF estimation.

\subsubsection{Sinusoidal Shaped Surface}

In [97], the fingerprint image is modeled as two-dimensional, sinusoidal shaped surface, and the frequency is approximated using the variation of the graylevel values orthogonal to the orientation together with a computed average amplitude.

\subsubsection{Further Methods}

A survey of further methods for ridge frequency estimation can be found in [154] and [103]. 


\subsection{Curved Regions}

In my opinion, the idea of $\mathrm{x}$-signature method suggests itself from the definition of the ridge frequency being the inverse of the inter-ridge distance. The advantages of the $\mathrm{x}$-signature are the simplicity of the idea and implementation and that can be computed very fast. However, the lower the image quality, the more likely it is to produce erroneous RF estimations. Again, this is the dilemma of fingerprint image enhancement: for those images that could profit the most from the enhancement, it is the most difficult to estimate the local context (orientation and ridge frequency) correctly. For this reason, there is a strong need for more robust RF estimation methods.

The averaging of gray-level values along the y-axis (see Figure 6.1) smooths the gray-level profile and in doing so it contributes to the robustness of the RF estimation. For this reason, it would be desirable to extend the oriented window in y-direction for improving the stability of the estimation. However, due to rectangular shape, this idea is only applicable, if the ridge lines are straight and parallel to each other. In general, fingerprint ridges and valleys are more or less curved, and therefore an elongated y-axis tends to cross neighboring ridges and valleys which introduces noise into the gray-level profile and consequently degrades the ridge frequency estimation (see Figure 6.8).

This limitation can be overcome by applying the following idea: instead of averaging gray-level values along straight lines, we adjust our lines to the curved ridges and valleys. A self-evident method for this purpose is the usage of quadratic Bezier curves (QBC) as proposed in Chapter 5. In the case of an orientation estimation without QBC, these curves can be approximated in piecewise linear steps.

A curved region centered in the pixel $\left(x_{c}, y_{c}\right)$ is computed in the following way: let $\theta_{c}$ be the orientation at $\left(x_{c}, y_{c}\right)$. Both orthogonal directions are $\theta_{o 1}=\theta_{c}+\frac{1}{2} \pi$ and $\theta_{o 2}=\left(\theta_{c}+\frac{3}{2} \pi\right)$ mod $2 \pi$. By going $n_{p}$ pixel steps of unit length into both directions, the start points for all $2 n_{p}+1$ curves are obtained. For each start point $\left(x_{s}, y_{s}\right)$, the decimal coordinates are rounded to the nearest pixel $\left(i_{s}, j_{s}\right)$ and the local orientation $\theta_{s}$ is looked up in the orientation image. First, the curve is approximated in direction $d_{1}=\theta_{s}$, afterwards these steps are repeated for direction $d_{2}=\theta_{s}+\pi$. Along each curve, the next point $\left(x_{i+1}, y_{i+1}\right)$ is computed from the previous: $x_{i+1}=x_{i}+\cos \theta_{i}$ and $y_{i+1}=y_{i}+\sin \theta_{i}$. For each curve point $\left(x_{i}, y_{i}\right)$, the gray value is estimated from the surrounding four pixels by bilinear interpolation [45]. In this way, the curves are mapped to twodimensional array (see Figure 6.4) from which the gray-level profile is calculated by averaging along the curves and convolution with a Gaussian kernel. Next, all local minima and maxima are detected. All the distances of consecutive minima or maxima in the range from 3 to 25 are regarded as valid, and the inverse of the median is stored as the ridge frequency estimation for the pixel $\left(x_{c}, y_{c}\right)$.

Very noteworthy is the visibility of the minutiae in the raw ridge frequency image (see Figure 6.6). This effect demonstrates the precision of the RF estimation and many applications are imaginable in order to make good use of 

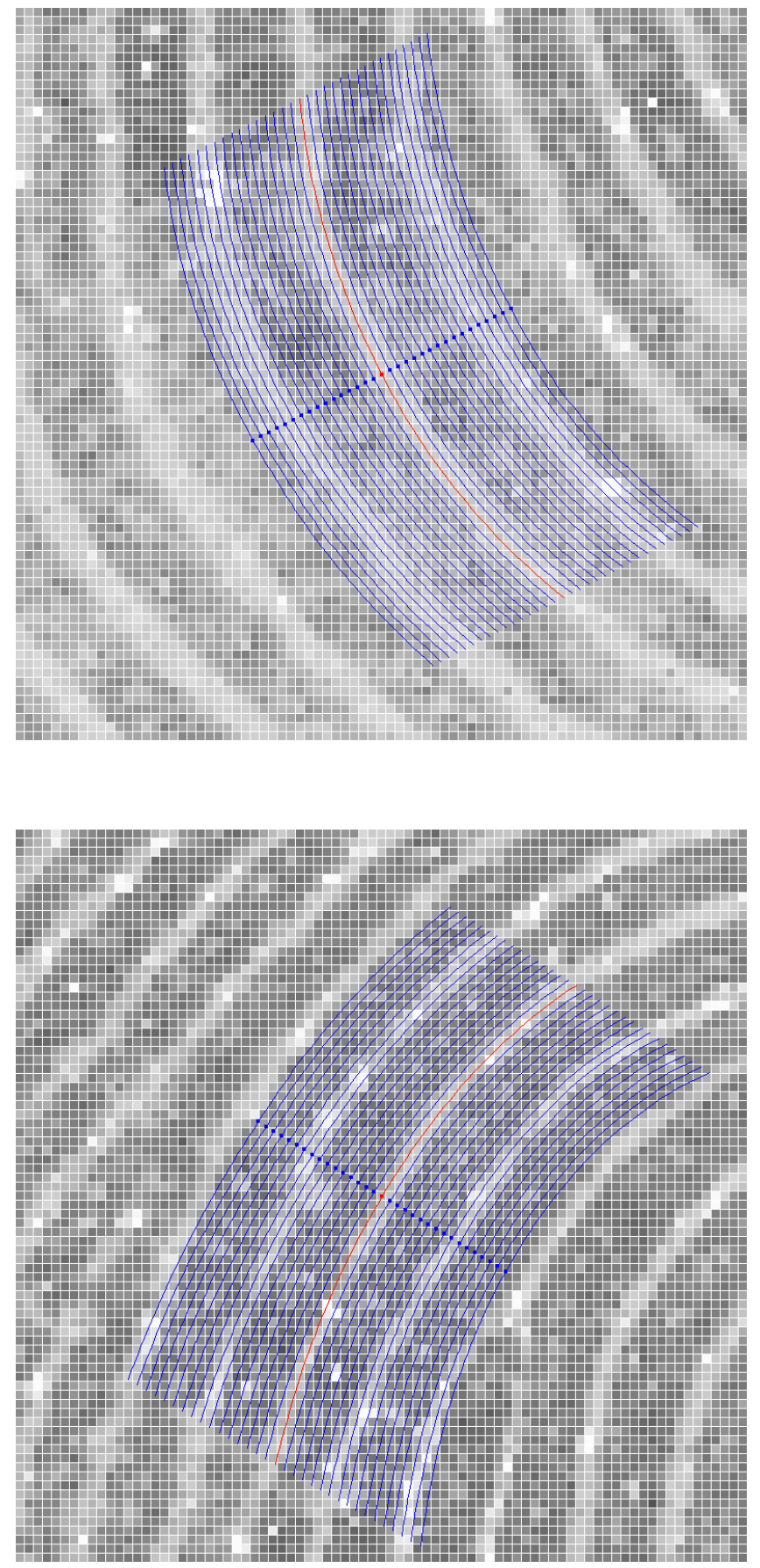

Figure 6.3: Both curved regions are centered at the red dot. Parallel curves start at the blue dots and are located on a line orthogonal to the ridge orientation at the center. Each curve consists of straight pieces which are obtained by following the local orientation for one pixel unit. 

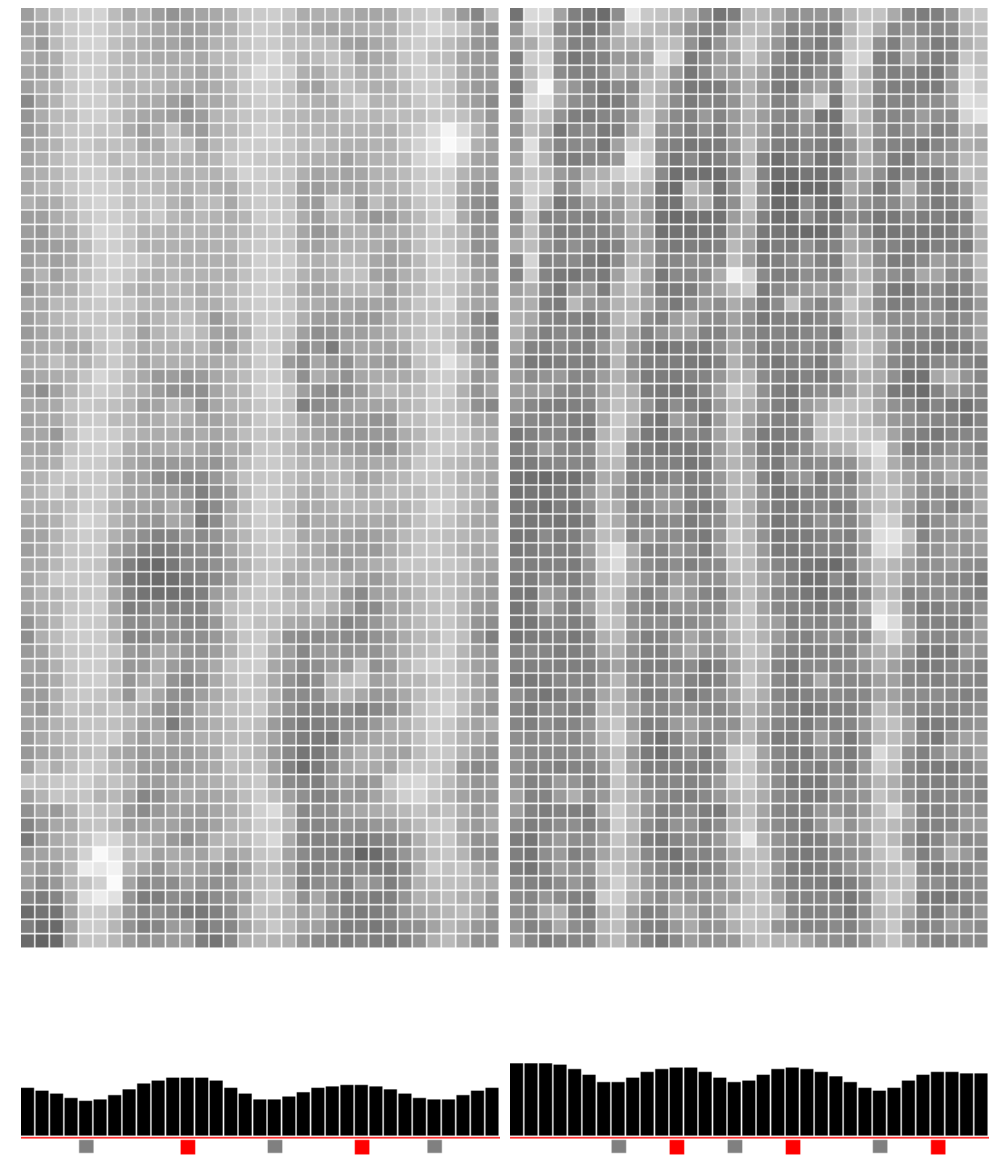

Figure 6.4: The bilinear interpolated gray values along the curves in Figure 6.3 are mapped to a matrix (top row). A column from top to bottom corresponds to a curve, the central column corresponds to the red curve in Figure 6.3. Below, the ridge and valley profile is obtained by averaging the gray values along each column and smoothing with a Gaussian kernel. Finally, local minima (gray square) and local maxima (red square) are detected. 

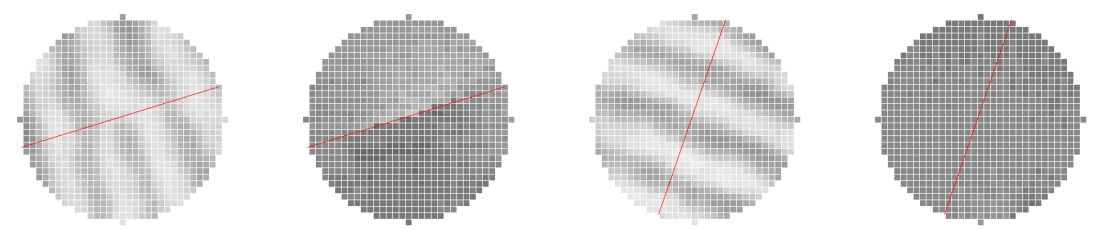

Figure 6.5: Two circular details from image 13_8 (left and third from left) of the FVC 2000 database 1 . The corresponding parts from the RF image are displayed right to the details. All circles are divided perpendicular to orientation into two halves. For left the detail, the mean inter-ridge distances are 10.2 pixels for the upper half and 8.4 pixels for the lower half. This gap is caused by the ridge ending in the center of the detail. The example on the right contains no minutiae and the mean inter-ridge distances are 8.2 and 8.4 pixels for the left and right half, respectively.

this piece of information. In my opinion, a thorough examination should be undertaken that clarifies how reliable minutiae can be directly extracted from the ridge frequency image. Moreover, the local properties of the RF image can be exceedingly useful for steering the curved Gabor filter which is presented in the next Chapter. In the proximity of a minutia location, the curved Gabor filter should proceed in a cautious way, i.e. limit the smoothing range along the local orientation, whereas in the absence of minutiae, it can operate with its full smoothing power. The RF image can also be applied for a retroactive inspection of minutiae which are detected by other methods, and for making decisions during the merging of minutiae templates. However besides minutiae, noise can also be a possible cause for peaks in the RF difference map. Therefore, the RF difference map should be applied in combination with a local quality map of the image. The Gabor filter should be tuned to smooth strongly in regions that are low quality or contain no minutiae, and it should be bridled in a neighborhood that is of medium to good quality and involves minutia points.

For the tests presented in Chapter 8 , the raw RF images were smoothed by simple averaging over a quadratic window as depicted in Figure 6.6. 

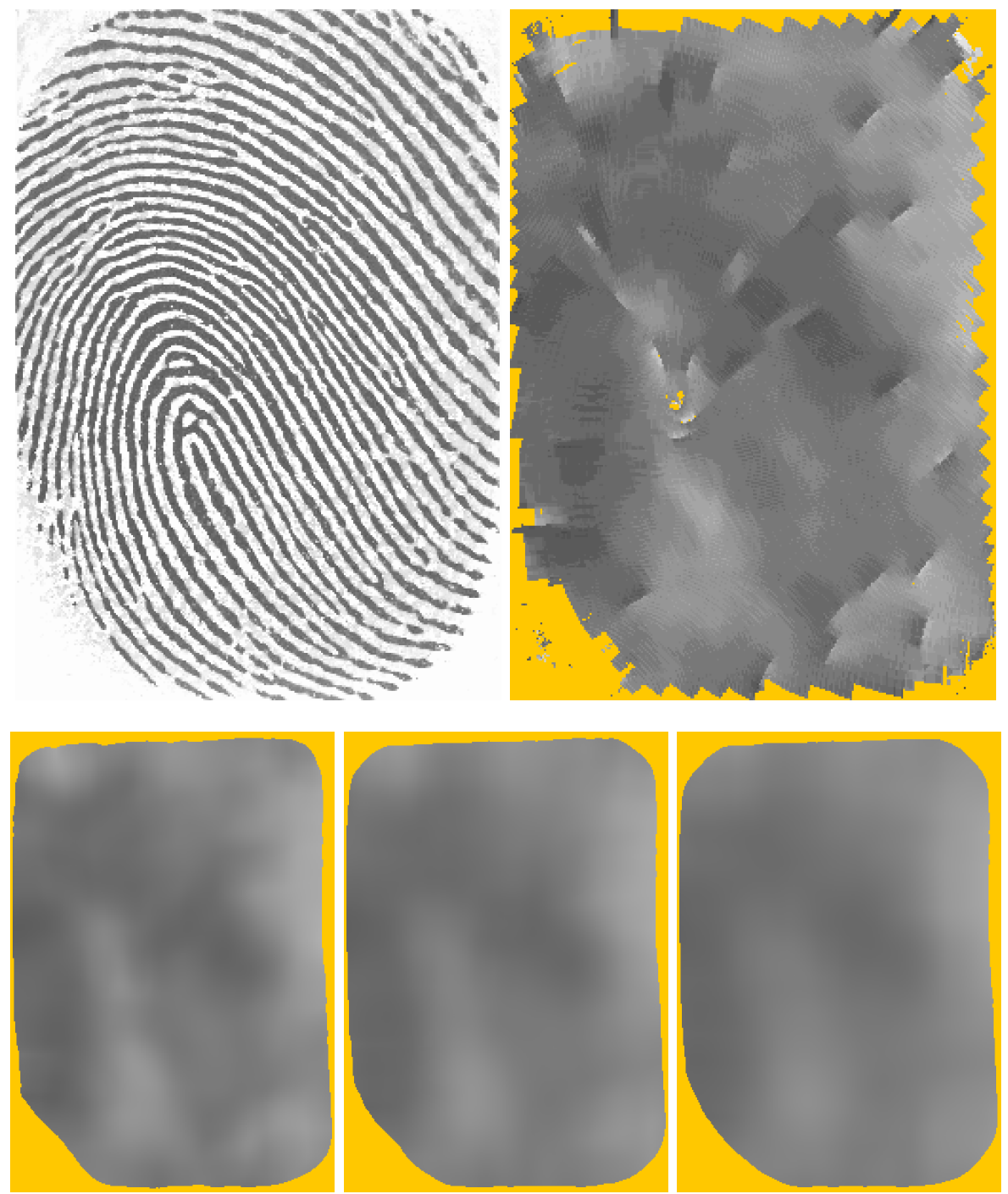

Figure 6.6: Top left: image 30_6 from FVC 2000 database 2; top right: the ridge frequency image. Light gray colors correspond to large inter-ridge distances, i.e. small frequencies. Dark gray colors indicate small inter-ridge distances, i.e. high frequencies. Ridge endings and bifurcations in the original image lead to edges in the frequency image which run orthogonal to the orientation. This enables the direct extraction of minutiae from RF image. Please note that the location and direction of the minutia can be estimated, but endings and bifurcations can not be distinguished. The bottom row displays mean smoothed frequency images. Smoothing window sizes are 33, 49 and 65 pixels from left to right. 


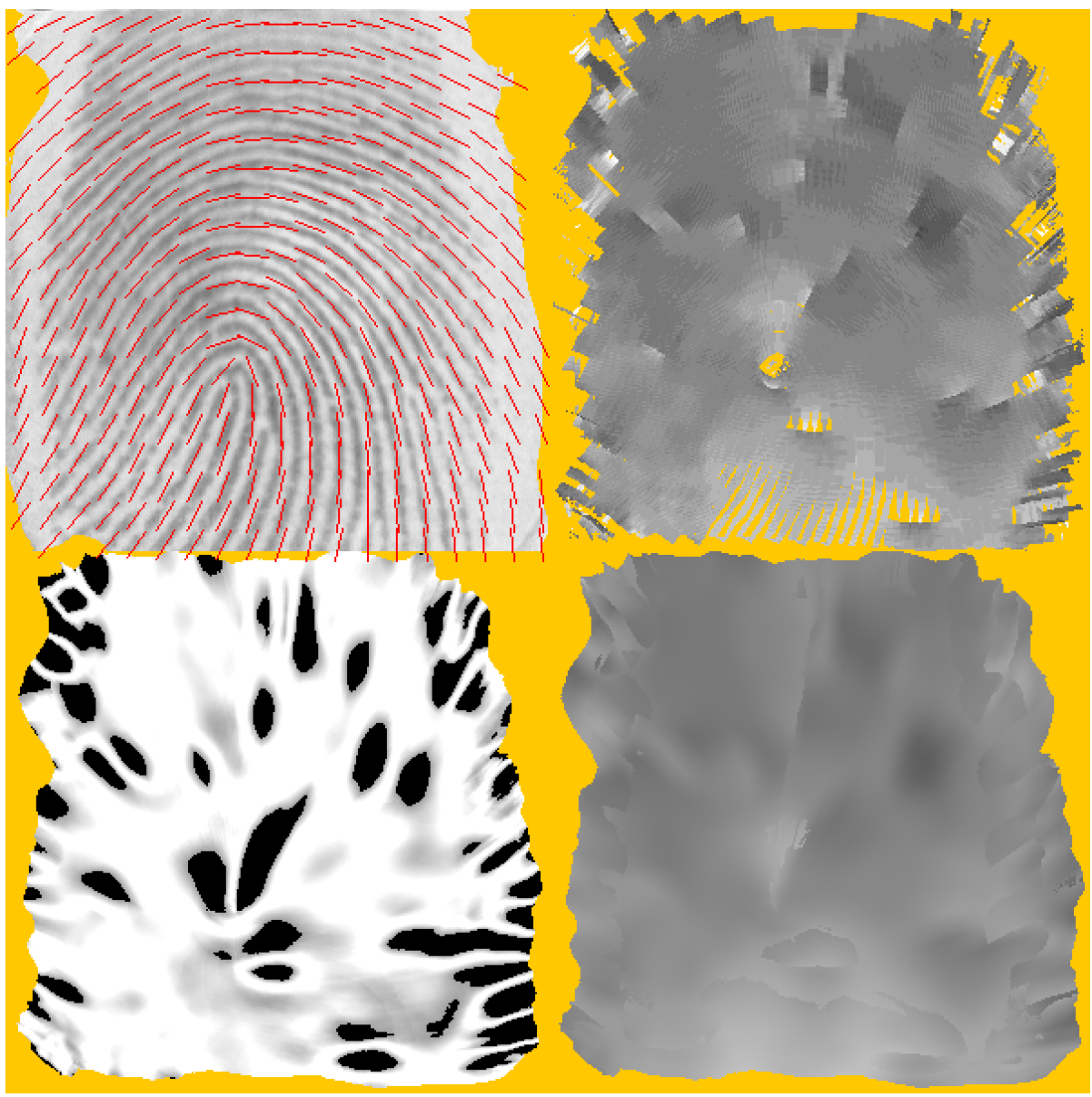

Figure 6.7: The orientation field (top left) and raw, pixelwise obtained ridge frequencies (top right) plotted for image 13_8 of FVC 2000 database 1. Bottom left, the RF differences computed according to Figure 6.5 are visualized. Differences below 0.4 pixels are drawn in white, values greater than 1.0 in black, and intermediate values correspondingly in gray. This RF difference map can be useful for verifying minutiae, steering the Gabor filter and further purposes. Bottom right, an alternative way of smoothing the RF image is shown. It was computed simultaneously with the difference image by setting the local RF to the mean value of the half circle which is closer to the mean of the full circle (see Figure 6.5). 

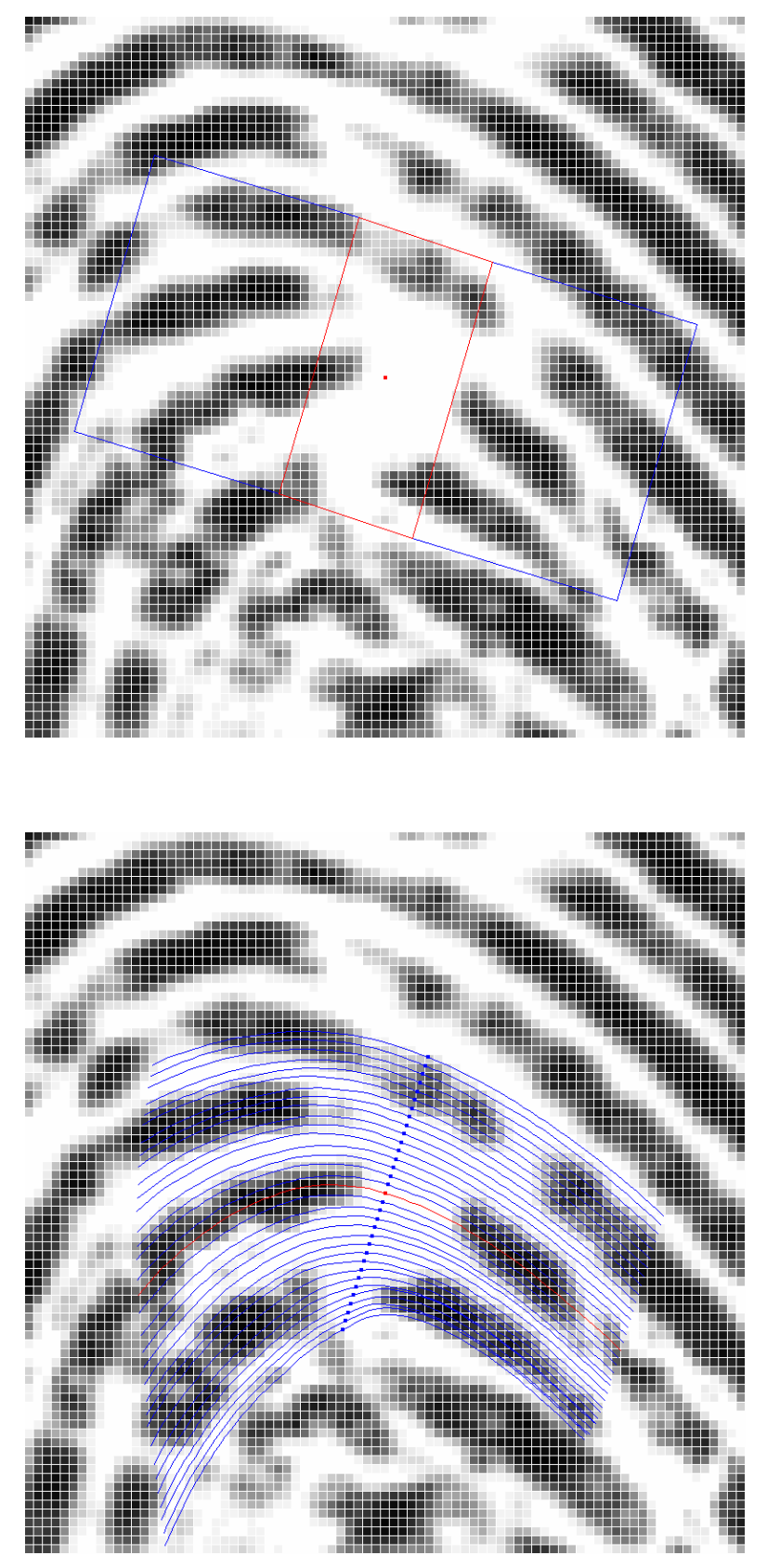

Figure 6.8: Comparing the $\mathrm{x}$-signature (top) and curved region (bottom) based methods for ridge frequency estimation. Noise can cause the $\mathrm{x}$-signature method to fail, because the oriented window (red rectangle) contains an insufficient amount of information. Magnifying the window (blue) along the local orientation does not remedy these deficiencies in regions of curvature and would lead to an erroneous gray level profile. The RF estimation based on curved regions overcomes these limitations by considering the change of local orientation, i.e. curvature. 


\section{Chapter 7}

\section{Image Enhancement}

The purpose of fingerprint image enhancement is to improve the overall performance of the fingerprint recognition system by optimally preparing input images for later processing stages. Most systems extract minutiae from fingerprints, and the presence of noise can interfere with the extraction. As a result, true minutia may be missed and false minutia may be detected which both has a negative impact on the recognition rate. In order to avoid these two types of errors, image enhancement aims at improving the clarity of the ridge and valley structure. The image quality is also an important factor for algorithms which extract other features like e.g. ridges [108] or apply correlation based techniques (see e.g. [12]).

With special consideration of the typical types of noise occurring in fingerprints, an image enhancement method should have four important properties:

- reconnect broken ridges e.g. caused by dryness of the finger or scars

- separate falsely conglutinated ridges e.g. caused by wetness of the finger or smudges

- preserve ridge endings and bifurcations

- robustness of the method to noise

The fourth point appears to be self-evident, but the robustness of the image enhancement method to noise is of special importance and should not be taken for granted. Many of the methods which can be found in the literature are error-prone and their performance tends to degrade in cases for which image enhancement matters the most: when dealing with low and very low quality images. Ironically, there is a substantial risk that the verification performance can be impaired by image enhancement (see Table 7.1 which cites the results from Table II in [38]).

For measuring the performance of the image enhancement and comparing different methods, it is important to have clear concept of the goal which shall be achieved by the image enhancement step. In this thesis, we will focus on enhancement methods that prepare the input image for algorithms which subsequently extract and match minutiae features [148]. In general, these methods 


\begin{tabular}{|c|c|c|c|c|}
\hline Enhancement method & DB1 & DB2 & DB3 & DB4 \\
\hline No enhancement & 14.5 & 9.5 & 6.2 & 7.3 \\
\hline Hong, Wan, Jain [60] & $(16.9)$ & 14.4 & 7.1 & 9.8 \\
\hline Chikkerur, Cartwright, Govindaraju [25] & $(19.1)$ & 11.9 & 7.6 & 10.9 \\
\hline Fronthaler, Kollreider, Bigun [38] & 12.0 & 8.2 & 5.0 & 7.0 \\
\hline
\end{tabular}

Table 7.1: EERs in \% of the NIST minutiae extractor and matcher for the original and enhanced images of FVC 2004 [101]. Parentheses indicate that only a small fingerprint area was useful for recognition. Results are cited from [38].

improve the clarity of the ridge and valley structure, and together with what can unequivocally be defined as noise (e.g. ridge interruptions caused by the dryness of the finger during scanning), they tend to remove sweat pores (see e.g.[67]), creases, scars, dots and incipients [24], so that these potential features will no longer be available in the enhanced image.

Most filters applied in fingerprint recognition systems are contextual filters [103] which means that the filter adapts its parameters and behavior according to the local context. As discussed in the previous chapters, the two most important types of contextual information are the local orientation and the local ridge frequency.

\subsection{Related Work}

A multitude of methods has been proposed for contextual filtering of fingerprint images. Among the first suggestions was a bank of drawn-out bell shaped filters created by rotation in 16 discrete directions [118]. For each pixel, one of the filters is chosen depending on the local orientation and convoluted with the image.

\subsubsection{Pyramid Methods}

Pyramid based techniques (see e.g. [39, 38] decompose the image into subbands which correspond to different scales. On each pyramid level, smoothing is performed. For choosing the orientation, a structure tensor [15] is applied whose usage was also suggested for coherence enhancing diffusion filtering [147].

\subsubsection{Short Time Fourier Transform}

In [25], the image is partitioned into blocks and for each block, a Fourier analysis is performed. As mentioned earlier, this method estimates the orientation and ridge frequency block-wise and utilizes this piece of information for filtering the image in the Fourier domain. In Chapter 8, the STFT method is included in the performance comparison of image enhancement approaches. 


\subsubsection{Discrete Cosine Transform}

An idea similar to [25] was presented in [81]. Again, the input image is partitioned into blocks and then transformed into the DCT domain. The estimations of the ridge frequency and orientation are followed by a filtering in the DCT domain with a modified Gabor filter. The authors compare the DCT method with a separable Gabor filter [6] in the spatial domain and the STFT method [25].

\subsubsection{Wavelet Transform}

A transform with a Daubechies wavelet basis decomposes the input image into a multi-resolution representation in [63]. The enhancement step is accomplished by convolution with quadrature mirror filters (QMFs) of eight coefficients.

\subsubsection{Gabor Filter}

The most popular technique for contextual filtering is the Gabor filter [41]. In the next section, we will discuss its properties and with respect to the specifics of fingerprints, an improvement in comparison to [60] is suggested.

\subsubsection{Anisotropic Filtering}

In $[49,50]$, anisotropic filters are proposed that are similar to the Gabor filter, but do not require a ridge frequency estimation. An ellipse shaped filter smooths the image pixel-wise along the ridges.

\subsubsection{Further Methods}

A survey of further fingerprint image enhancing methods is given in [3] and [103].

\subsection{Gabor Filter}

The Gabor filter is a two-dimensional, linear filter formed by the combination of a cosine with a two-dimensional Gaussian function and it has the general form:

$$
\begin{gathered}
g\left(x, y, \theta, f, \sigma_{x}, \sigma_{y}\right)=\exp \left\{-\frac{1}{2}\left[\frac{x_{\theta}^{2}}{\sigma_{x}^{2}}+\frac{y_{\theta}^{2}}{\sigma_{y}^{2}}\right]\right\} \cdot \cos \left(2 \pi \cdot f \cdot x_{\theta}\right) \\
x_{\theta}=x \cdot \cos \theta+y \cdot \sin \theta \\
y_{\theta}=-x \cdot \sin \theta+y \cdot \cos \theta
\end{gathered}
$$

Here, the Gabor filter is centered at the origin. $\theta$ denotes the rotation of the filter related to the x-axis and $f$ the local frequency. $\sigma_{x}$ and $\sigma_{y}$ signify the standard deviation of the Gaussian function along the $\mathrm{x}$ - and $\mathrm{y}$-axis, respectively. The Gabor filter is a local operator which is applied to each pixel of the image 

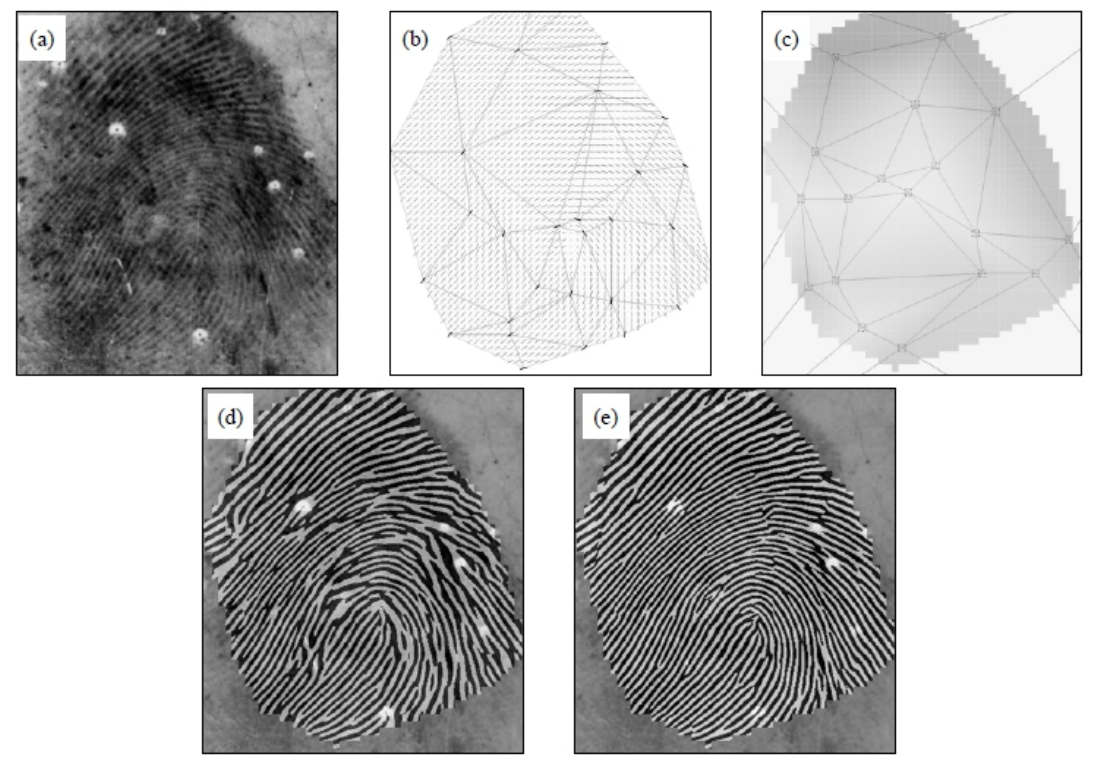

Figure 7.1: Screenshot from [19]. (a) displays a latent fingerprint from NIST special database 27 [43]. (d) and (e) show the result of image enhancement with a Gabor filter. In (d), the orientation field and ridge frequency image was estimated automatically as described in [60]. For (e), the orientation field (b) and ridge frequency $(\mathrm{c})$ were marked by a human expert.

foreground. It considers the gray values of the pixels in a predefined neighborhood and its parameters for the aim of producing an enhanced output pixel. The Gabor filter has the beautiful property of simultaneously minimizing the uncertainty in space and spatial frequency [29, 85].

\subsubsection{Filter Parameters and Their Impact}

The four Gabor filter parameters have a decisive influence on the behavior of the filter. Two of them, the local orientation $\theta$ and the local ridge frequency $f$ are estimated prior to the filter application. The standard deviations of the Gaussian envelope $\sigma_{x}$ and $\sigma_{y}$ are tuning parameters which steer the range of included neighboring pixels.

Local Orientation and Ridge Frequency The local orientation $\theta$ plays a key role for the Gabor filter. Orientation estimation errors tend to create spurious minutiae and ultimately deteriorate the verification performance. Illustrations of this effect are given in Figure 7.2 and 7.1. Additional examples can be found in [19] and [140].

The correctness and precision of the local ridge frequency is of a great importance as well, because the Gabor filter creates a ridge structure with the specified input frequency. Underestimating the actual ridge frequency (i.e. overestimating the inter-ridge distance) will cause the deletion of ridge lines, and the inverse 

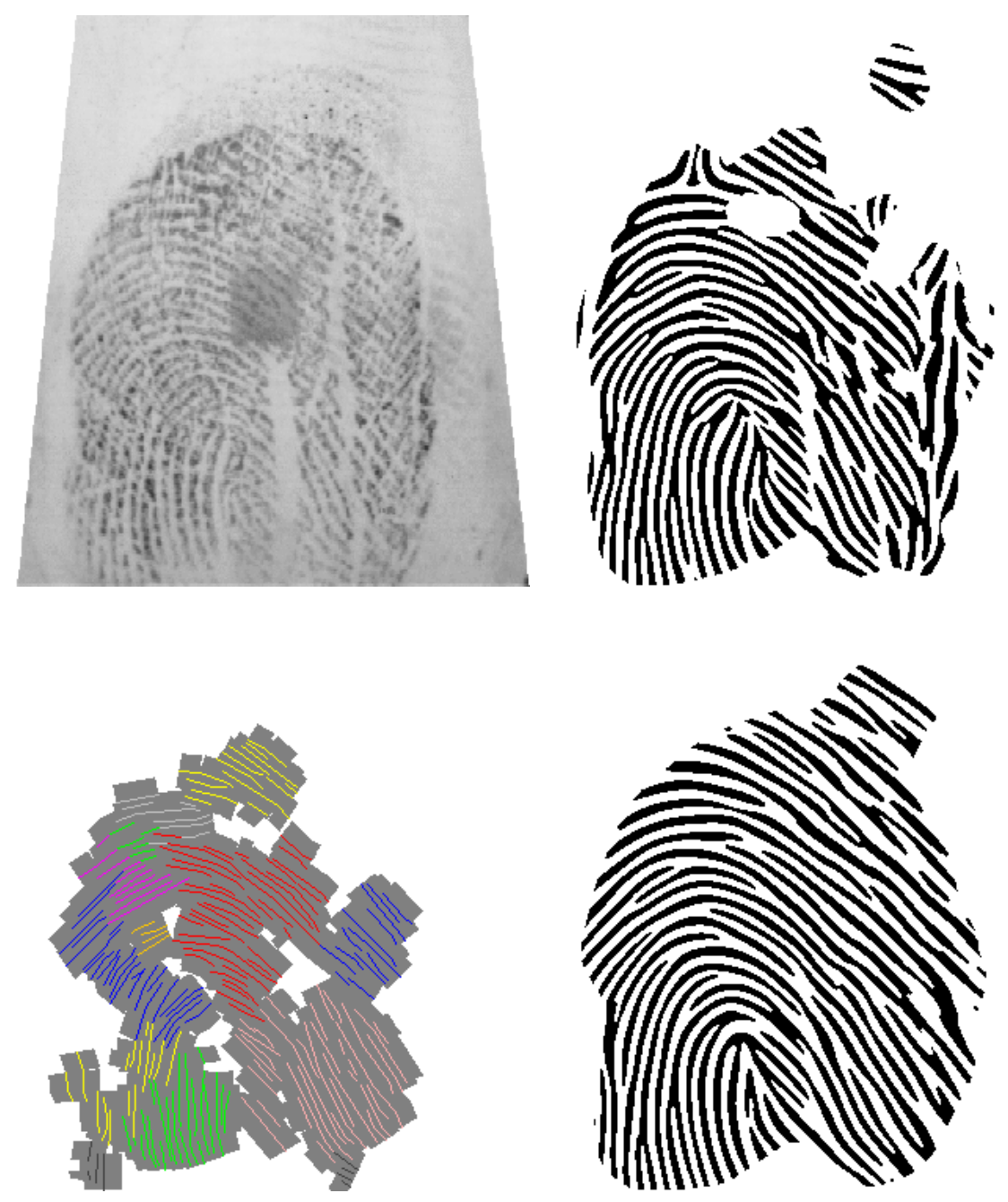

Figure 7.2: Top left: image 107_3 from FVC 2004 database 2. On the top right, the original image was contextually filtered and binarized by the VeriFinger 5.0 SDK. Evidently, artifacts are caused by errors in the orientation estimation. If instead the line sensor method with tracing is applied for the OF estimation (illustration of the merged coherend structure on the bottom left) and the original image is smoothed along the orientation, the binarization using the VeriFinger 5.0 SDK leads to an improved output. 
situation of overestimating the real ridge frequency will induce the creation of false ridges. Figure 6.2 depicts the consequences of defective ridge frequency estimations for both cases. These effects are also visible when comparing images (d) and (e) in Figure 7.1.

In summary, accurate estimations of the local orientation and ridge frequency are a conditio sine qua non for the application of the Gabor filter. As extensively discussed in Chapter 4 and 6 , the steadiness of these estimations are a challenge for low quality fingerprint images. The authors of [19] argue likewise and suggest to manually mark the orientation and frequency for the enhancement of very low quality images like latents. Here, I would like emphasize that I do not share this pessimistic point of view. The previously described methods for OF and RF estimation achieve strong improvements in comparison to currently widely used methods like the gradients based OF estimation and the x-signature for RF estimation.

In Section 7.3, we will introduce the novel idea of a curved Gabor filter and in Chapter 8 an architecture for multi-level judgment aggregation. In my opinion, the combination of these four factors provides an excellent tool kit for tackling low and very low quality images.

Standard Deviations $\sigma_{x}$ and $\sigma_{y}$ The choice of $\sigma_{x}$ and $\sigma_{y}$ steers the range of influence along the local orientation and in orthogonal direction. This property corresponds to the extension of the oriented window (see Figure 6.1) of the x-signature. Larger values of both parameters increase on the one hand, the capability to remove noise, and on the other hand, the probability of the creating spurious features. Again, this effect is caused by the rectangular shape of the Gabor filter window and the often curved course of fingerprint ridges. For smaller $\sigma_{x}$ and $\sigma_{y}$ values, the filter tends to create fewer spurious features, but also become less effective in improving the ridge structure. So, the choice of $\sigma_{x}$ and $\sigma_{y}$ boils down to a trade-off between these two extremes. The authors of [60] suggest $\sigma_{x}=4.0$ and $\sigma_{y}=4.0$ based on their experiences. In [19], $\sigma_{y}$ is chosen to be larger than $\sigma_{x}$ which is defined to have a specified number of peaks independently of the ridge frequency: $\sigma_{x}=\frac{3.4}{9 \cdot f}, \sigma_{y}=\frac{4}{3} \sigma_{x}$.

Illustrations showing how different values of $\sigma_{x}$ and $\sigma_{y}$ influence the enhanced image are given in Figure 7.4.

\subsubsection{Gabor Filter Modifications}

Some modified versions of Gabor filters can be found in the literature. The authors of [152] argue that the cosine function taking effect orthogonal to the orientation does not map the real ridge profile in many cases and they propose an adaptation of the filter to the local gray-level profile.

Some experimentations with adaptive Gabor filter parameters applied on partial images from FVC 2000 were presented in [44]. A separable Gabor filter was introduced in [6] for a reduction of the computing time. 
Carrying on the work of [60], a Gabor filtering method with alterations is suggested in [158]: the first modification is the use of circular instead of rectangular shaped windows for the Gabor filter in order to avoid the creation of aforementioned artifacts. And secondly, an average ridge frequency is estimated for the complete image foreground. Comparing their altered method with the Gabor filter from [60] on 14 images, the authors observe an improvement. In my opinion, it seems natural to apply a circular mask instead of rectangle, but the influence of the excluded pixels is because of the Gaussian kernel so close to zero that the gain should be negligible. In regions with bending ridges, the circular filter can still cross neighboring ridges, depending on the size of the mask and the local inter-ridge distance. The data basis of 14 images is too small to draw any conclusions, but in my opinion any possible improvements are achieved by using a globally averaged ridge frequency. This speaks volumes about the quality of the $\mathrm{x}$-signature based RF estimation and underlines the demand for a robust RF estimation method as discussed in the previous chapter. Moreover, in many images there is a huge disparity of local ridge frequency from one image region to another. The use of a global frequency value remains unsatisfactory and contradicts the idea of contextual filtering.

Gabor filter banks are also used for measuring texture energy which is called "Gabor features" [76]. For fingerprint images, these Gabor features can e.g. be employed for singular point detection [90], for image segmentation [5] and for quality estimation [136]. Another example are the well-known FingerCodes [75].

\subsection{Curved Gabor Filter}

Bearing in mind the specifics of fingerprints, we introduce a curved Gabor filter (see Figure 7.3) which is bent according to the degree of local ridge curvature. In this way, we overcome all aforementioned limitations that were imposed by the rectangular shape of the filter window. Especially, a strong smoothing along the ridges for the reconnection of broken ridges and the separation of falsely conglutinated ridges (see Figure 7.4) without creating spurious features is enabled by the curved Gabor filter. A comparison of image enhancement by the Gabor filter as defined in [60] and the curved Gabor filter is depicted in Figures 7.6 to 7.11 .

In this thesis, we apply the curved regions (see Figure 6.3) from the previous chapter for building the curved Gabor filter. In principal, the quadratic Bezier curves from Chapter 5 could also be utilized for this purpose. The reuse of the curved regions has the additional advantage of saving computational time. For estimating the ridge frequency, the curved region is mapped to a twodimensional array of bilinear interpolated gray values (see Figure 6.4). Exactly the same array will be accessed for curved Gabor filter. Further acceleration is to be expected using the GPUs of the graphics card. An implementation of the fast line sensor, the curved ridge frequency estimation and the curved Gabor filter for GPUs (e.g. using Nvidia's CUDA ${ }^{1}$ technology) is one of the goals for the near future.

${ }^{1}$ http://developer.nvidia.com/object/cuda.html 


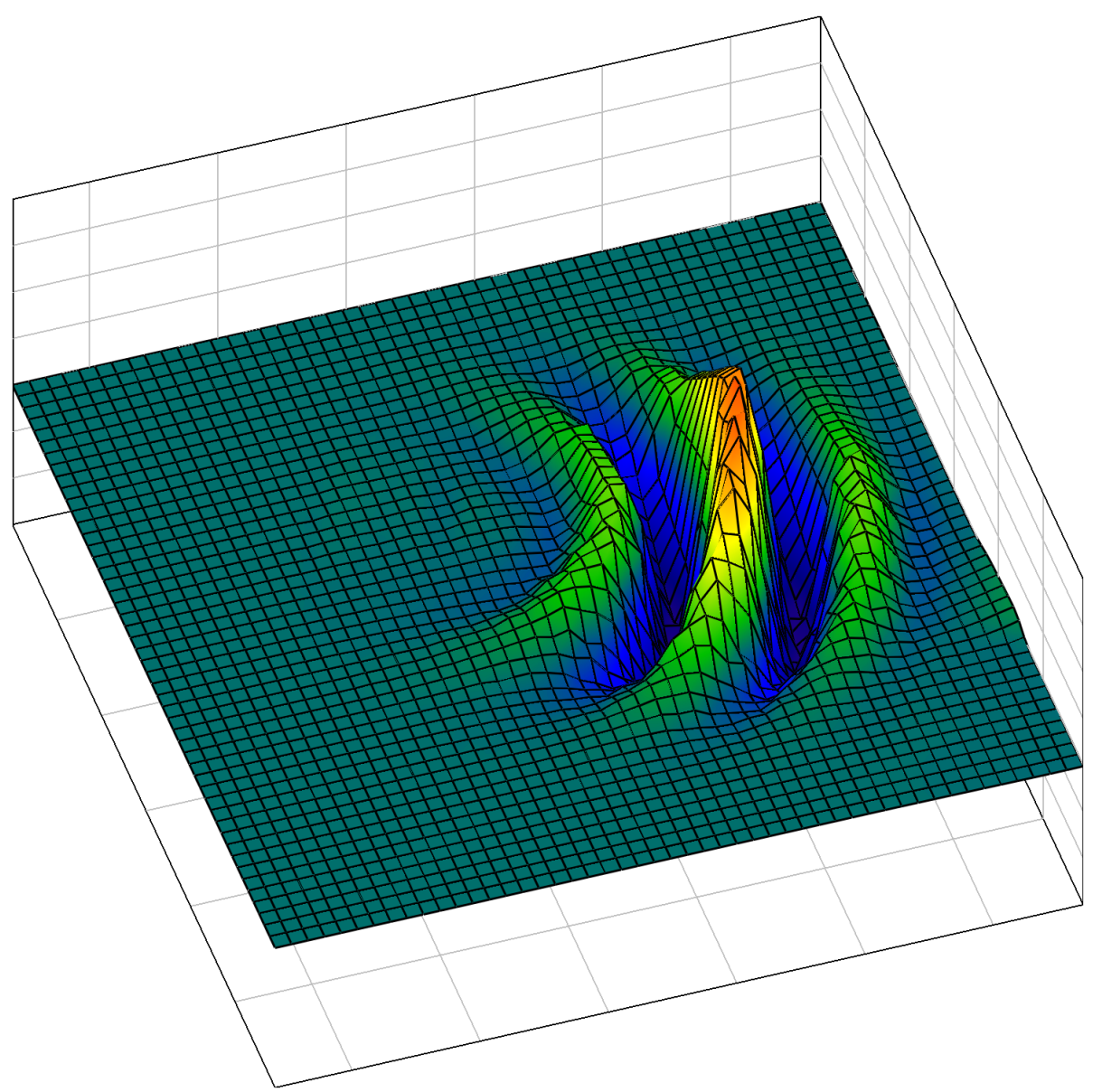

Figure 7.3: Visualization of a curved Gabor filter for a pixel near a core point. Filter parameters are: $\sigma_{x}=6.0, \sigma_{y}=8.0$. The underlying curved region consists of 33 parallel curved lines with 65 points for each line. 

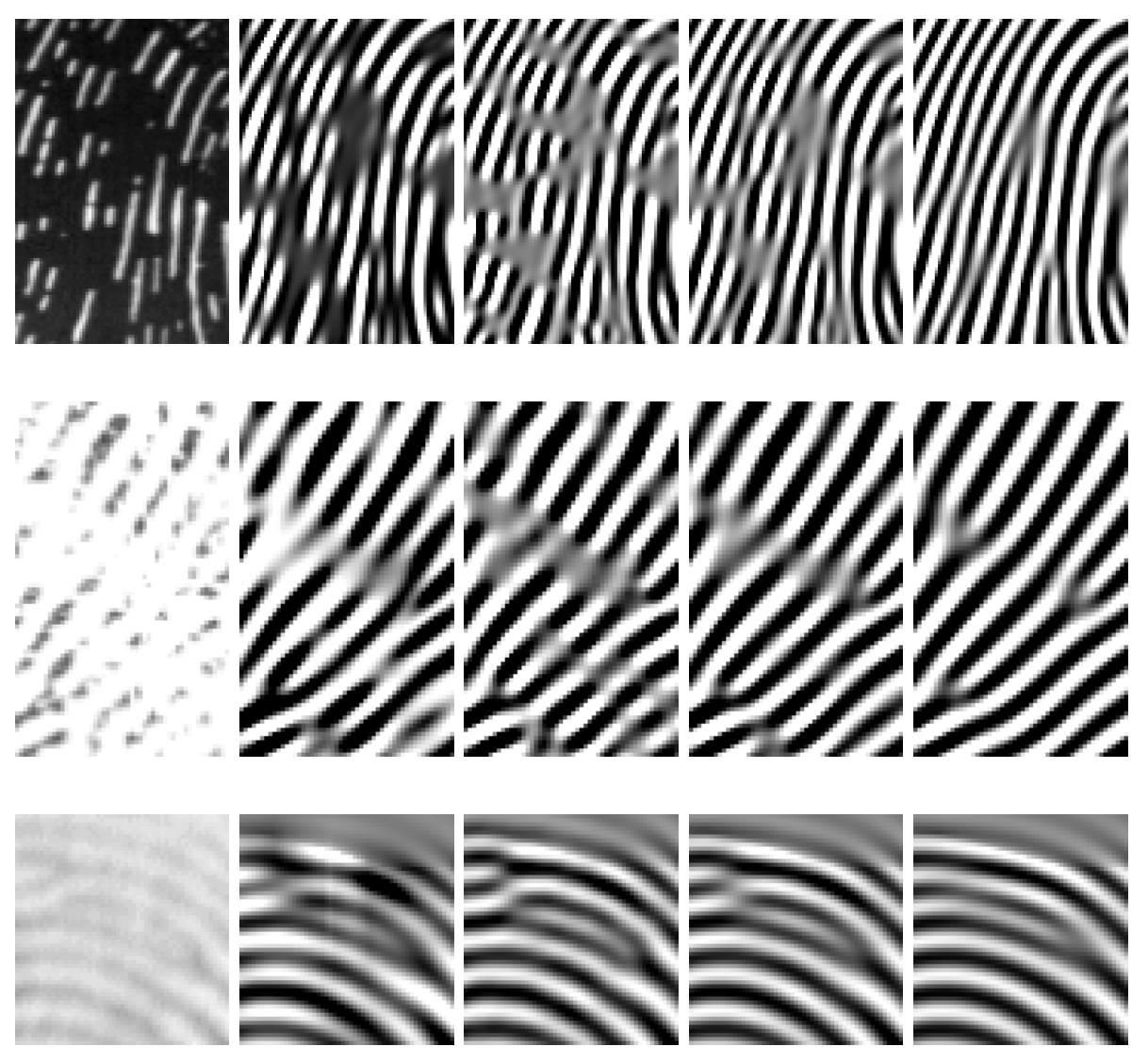

Figure 7.4: From top to bottom: details from images 10_8 and 107_2 of FVC2004 database 1 and image 21_5 of FVC2000 database 1. The left column displays details of the original images and aside, the corresponding parts from enhanced versions applying the curved Gabor filter with the following parameters, from left to right: $\sigma_{x}=2.0, \sigma_{y}=4.0 ; \sigma_{x}=4.0, \sigma_{y}=2.0 ; \sigma_{x}=4.0, \sigma_{y}=4.0$; $\sigma_{x}=6.0, \sigma_{y}=8.0$; In the top row, the example on the right demonstrates in particular the usefulness of the curved Gabor filter with large $\sigma_{y}$ values for separating falsely conglutinated ridges. The images in the middle row illustrate the capabilities of the curved Gabor filter to reconnect interrupted ridges (especially on the right, by applying large $\sigma_{y}$ values). The example at the bottom row was chosen in order to create an awareness for the dangers of smoothing. Depending the specific configuration of ridges and the precision of the local orientation estimation, it is possible that the smoothing step alters a bifurcation into an ending, and vice versa. In this particular case, two bifurcations switched to opposing endings that were connected for the filter with the largest $\sigma_{y}$ value. 

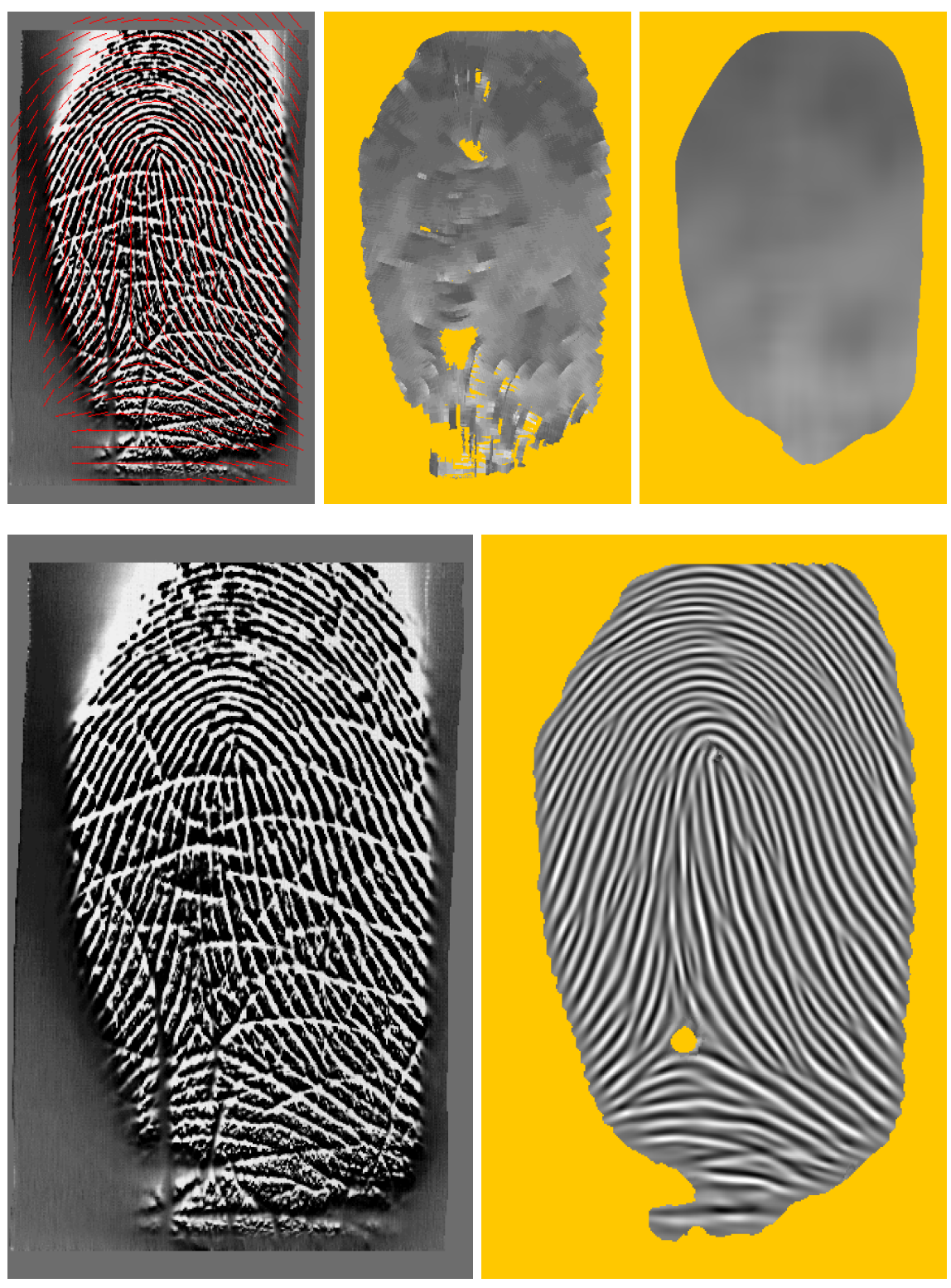

Figure 7.5: Enhancement with curved Gabor filter. Overview for image 10_1 of FVC2004 database 3. Top row from left: line sensor OF, raw RF image and smoothed RF image. Bottom right: enhanced with filter parameter $\sigma_{x}=3.0$ $\sigma_{y}=7.0$. The same OF and smoothed RF image is passed on to all filters. 

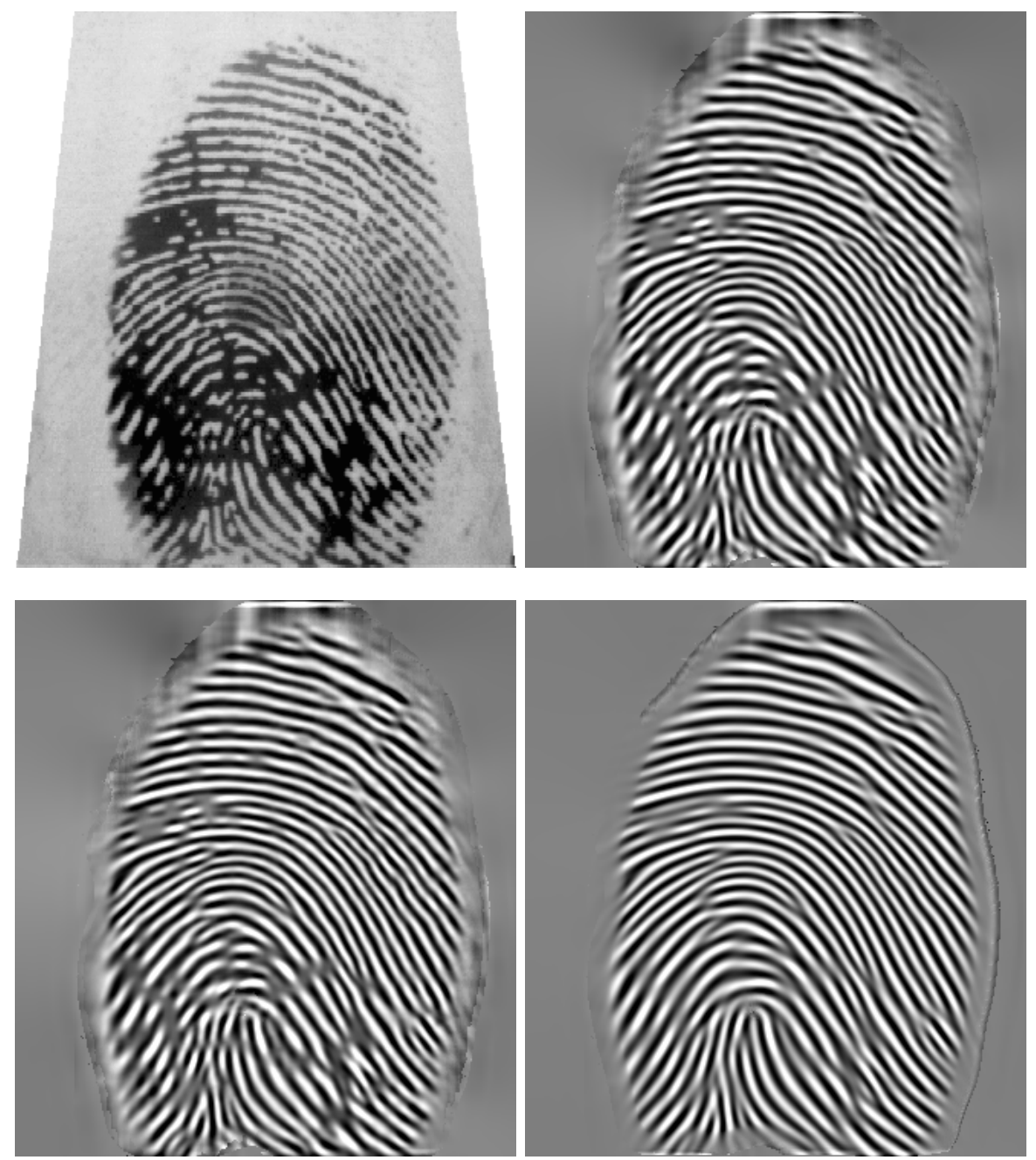

Figure 7.6: Comparing the enhancement of image 69_7 of FVC 2004 database 2 using the Gabor filter as defined by Hong et al. in [60] (top right and bottom left) and the curved Gabor filter (bottom right). Filter parameters are $\sigma_{x}=4.0$, $\sigma_{y}=4.0$ for top right, $\sigma_{x}=4.0, \sigma_{y}=7.0$ for the bottom row. The same OF and smoothed RF image is passed on to all filters. 

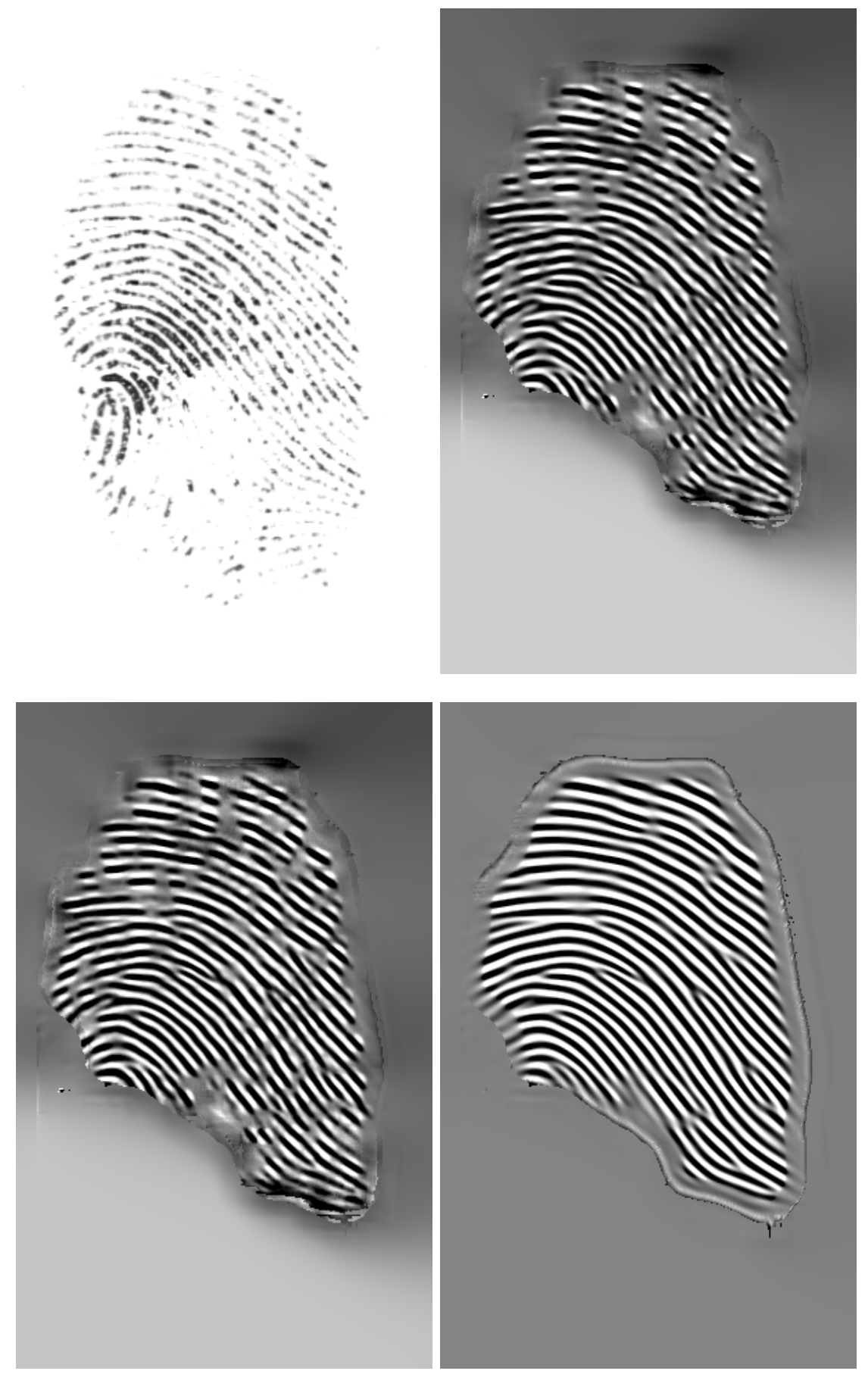

Figure 7.7: Comparing the enhancement of image 105_1 of FVC 2004 database 1 using the Gabor filter as defined by Hong et al. in [60] (top right and bottom left) and the curved Gabor filter (bottom right). Filter parameters are $\sigma_{x}=4.0$, $\sigma_{y}=4.0$ for top right, $\sigma_{x}=4.0, \sigma_{y}=7.0$ for the bottom row. 

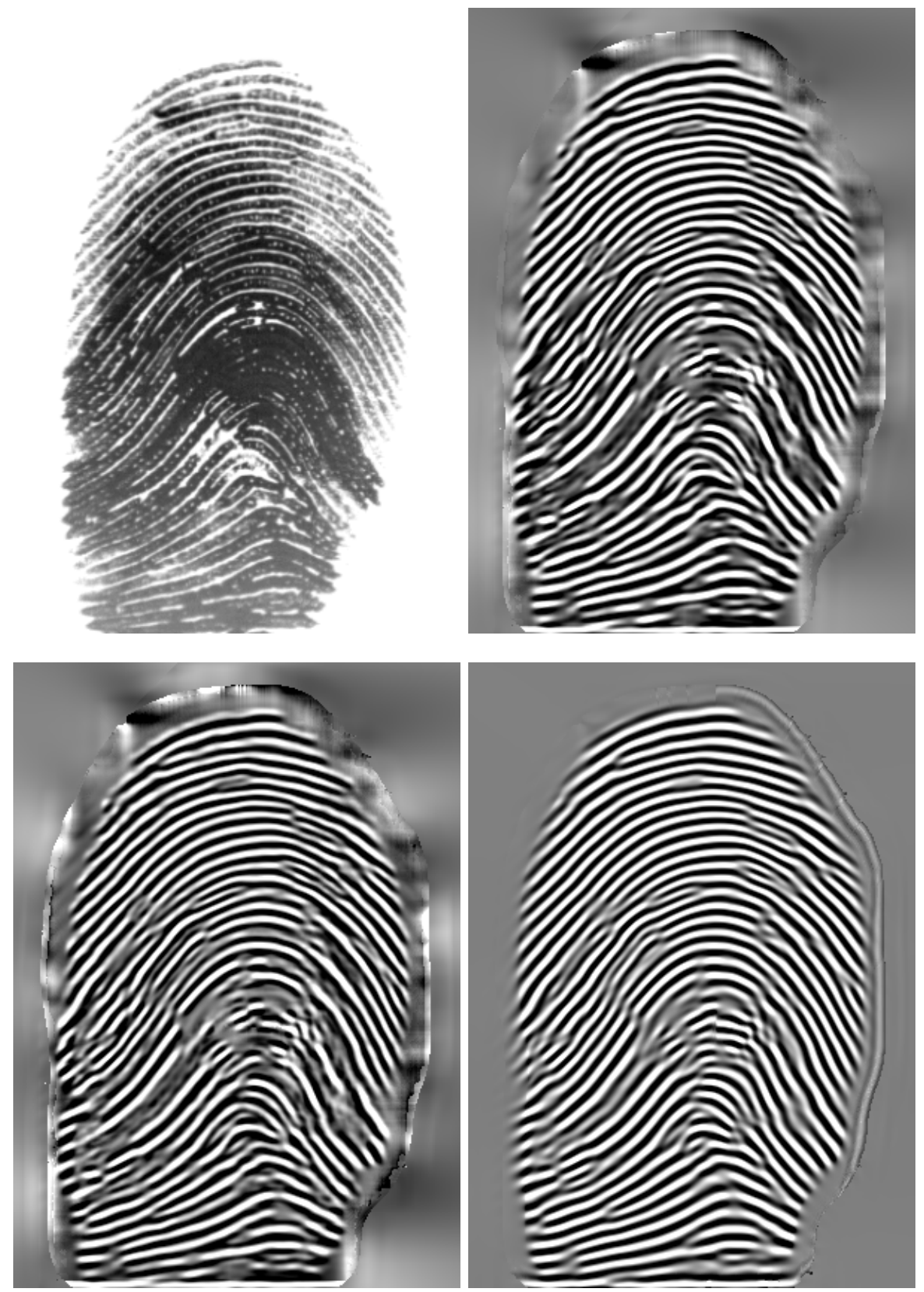

Figure 7.8: Comparing the enhancement of image 100_8 of FVC 2004 database 1 using the Gabor filter as defined by Hong et al. in [60] (top right and bottom left) and the curved Gabor filter (bottom right). Filter parameters are $\sigma_{x}=4.0$, $\sigma_{y}=4.0$ for top right, $\sigma_{x}=7.0, \sigma_{y}=4.0$ for the bottom row. The same OF and smoothed RF image is passed on to all filters. 

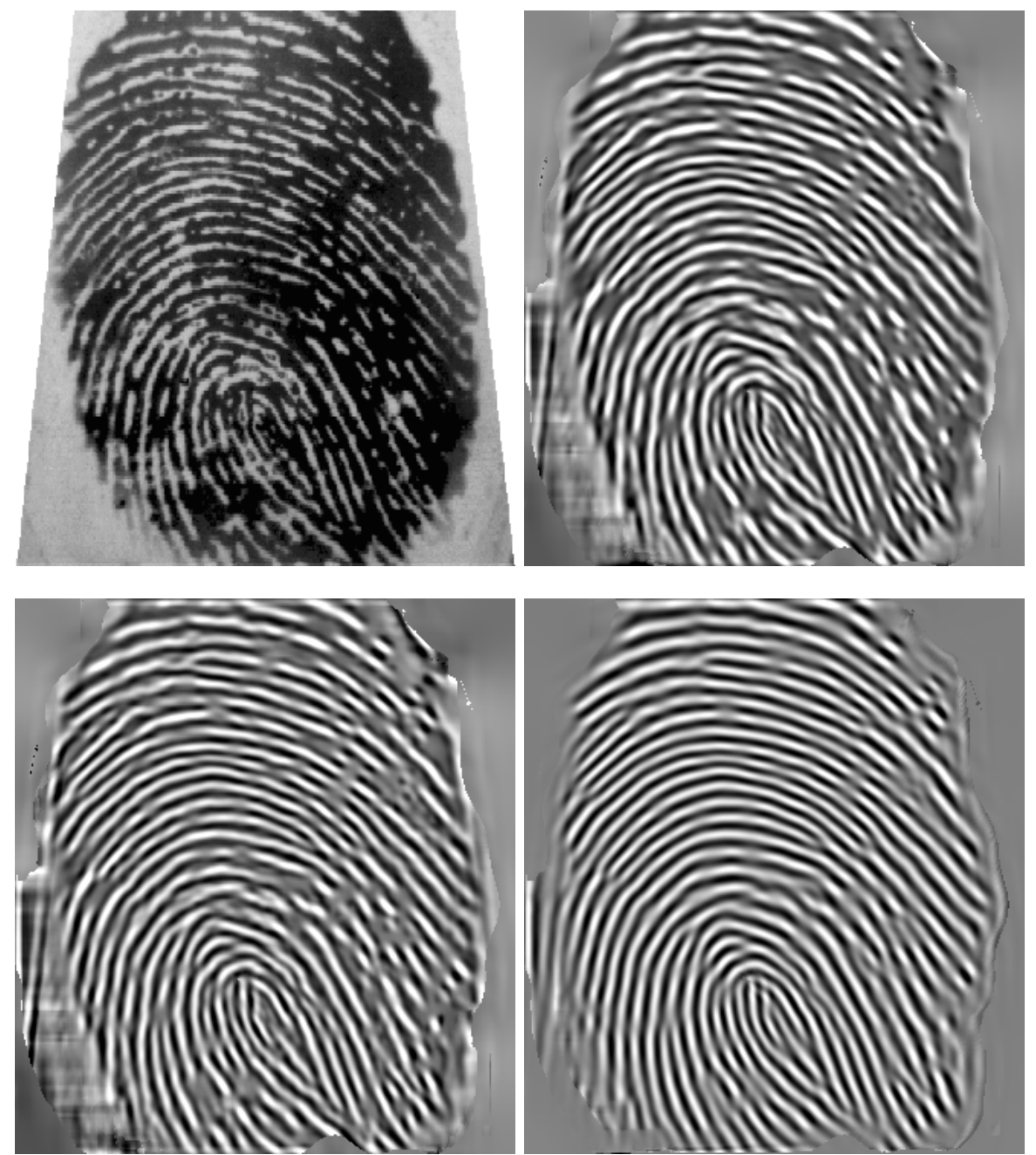

Figure 7.9: Comparing the enhancement of image 102_8 of FVC 2004 database 2 using the Gabor filter as defined by Hong et al. in [60] (top right and bottom left) and the curved Gabor filter (bottom right). Filter parameters are $\sigma_{x}=4.0$, $\sigma_{y}=4.0$ for top right, $\sigma_{x}=7.0, \sigma_{y}=4.0$ for the bottom row. The same OF and smoothed RF image is passed on to all filters. 

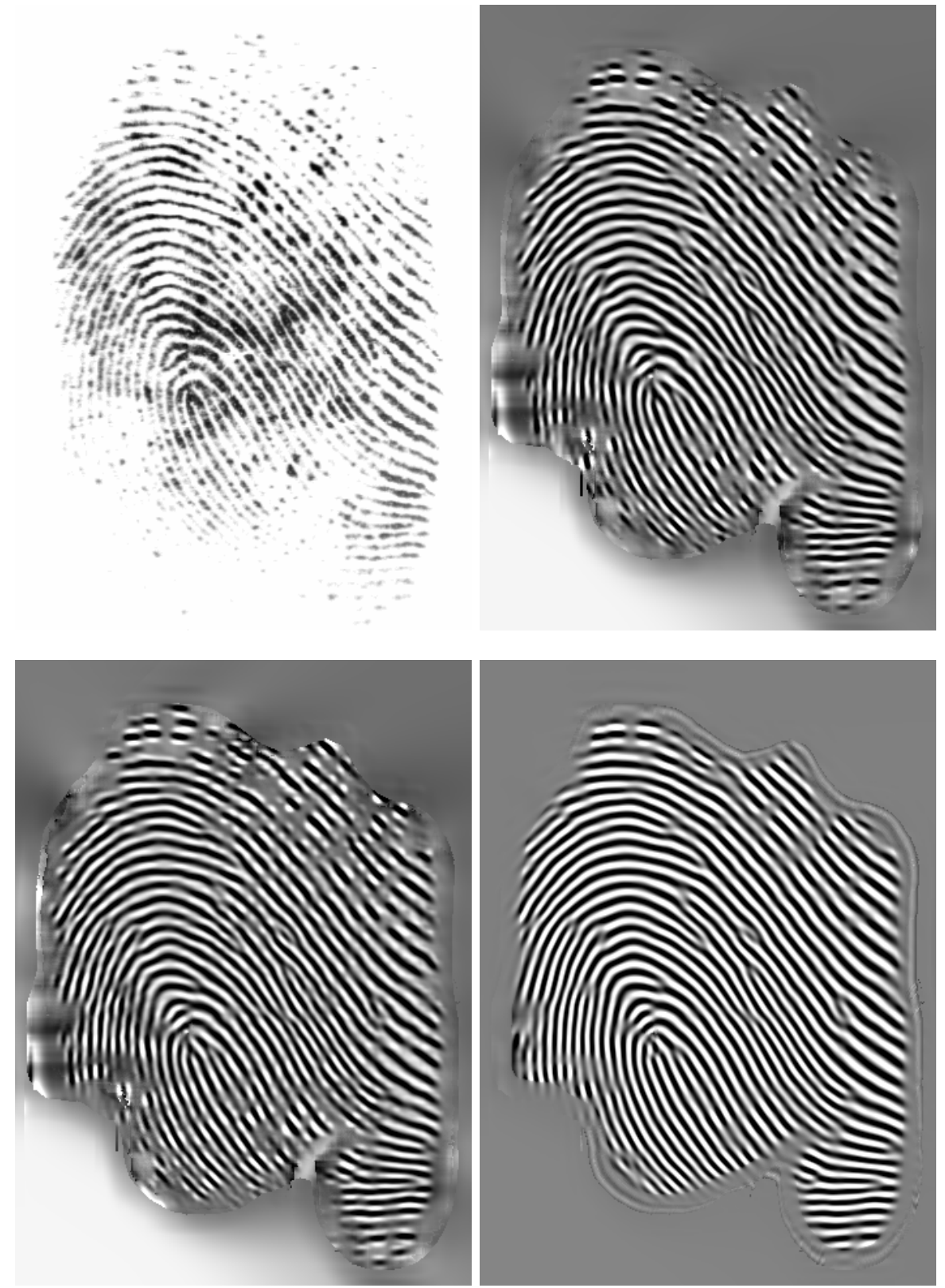

Figure 7.10: Comparing the enhancement of image 106_7 of FVC 2004 database 1 using the Gabor filter as defined by Hong et al. in [60] (top right and bottom left) and the curved Gabor filter (bottom right). Filter parameters are $\sigma_{x}=4.0, \sigma_{y}=4.0$ for top right, $\sigma_{x}=7.0, \sigma_{y}=4.0$ for the bottom row. The same $\mathrm{OF}$ and smoothed RF image is passed on to all filters. 

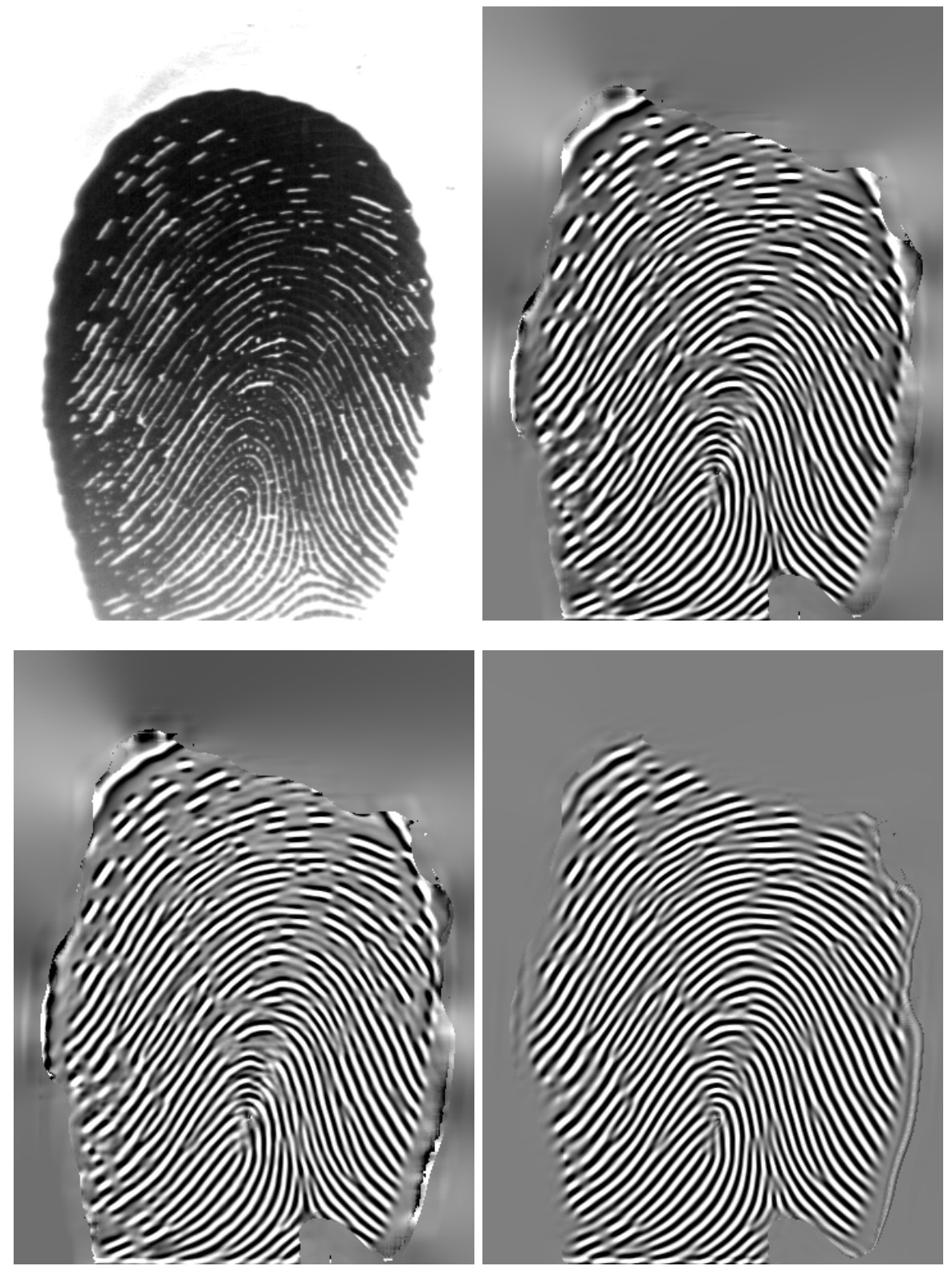

Figure 7.11: Comparing the enhancement of image 16_8 of FVC 2004 database 1 using the Gabor filter as defined by Hong et al. in [60] (top right and bottom left) and the curved Gabor filter (bottom right). Filter parameters are $\sigma_{x}=4.0$, $\sigma_{y}=4.0$ for top right, $\sigma_{x}=4.0, \sigma_{y}=7.0$ for the bottom row. The same OF and smoothed RF image is passed on to all filters. 


\section{Chapter 8}

\section{Multi-Level Judgment Aggregation}

Fusion is the concept of combining different sources of information in order to improve the performance of a biometric system. Depending on the type of information that is combined, customarily the following kinds of fusion are distinguished:

- Multiple modalities [130], e.g. face, iris and fingerprint

- Multiple units, e.g. left iris and right iris, or right index finger and right thumb

- Multiple sensors, e.g. different cameras for face, or an optical and a capacitive sensor for fingerprints

- Multiple samples, e.g. two prints of the same finger

- Multiple algorithms, e.g. two different extractors and matchers

In this thesis, we focus on the last-mentioned item on the list and consider the case that we have one sample (image) of one unit (finger) of one modality (fingerprint) captured by one sensor. For this scenario, the following fusion categories are covered in the literature:

- Decision-level fusion

- Rank-level fusion

- Score-level fusion

Decision-level fusion (see e.g. [141]) is the combination of binary decisions made by different matchers. Established combination rules are AND, OR, majority voting and weighted majority voting. On all levels of information fusion, the danger exists that the decision based on fusion can impair the performance of the biometric system in comparison to the best individual matcher. For decisionlevel fusion, this aspect was pointed out in [30]. 
Rank-level fusion (see e.g. [113]) can be applied, if the task of the system is identification (opposed to verification which is the main focus of this chapter). For a given query, each algorithm outputs a list of potential identities which are sorted by the computed similarity to the query. The highest similarity is assigned to the lowest rank. A rank-level fusion which was originally proposed for political voting is called Borda count. The combined ranks are obtained by summing up the individual ranks for each biometric identity or political candidate. It was suggested by the French mathematician Jean-Charles de Borda. A modified version of Borda count is used at the Eurovision Song Contest, in which the top ranked country wins 12 points, the second ranked 10 points, the third ranked 9 points and so on.

Score-level fusion is the most popular type of fusion. In general, fusion techniques can be divided into three categories.

- Score density-based methods (see e.g. [115] and [114])

- Classifier-based methods

- Transformation-based methods

Transformation-based methods for score fusion consist of two steps: in a preparation step, scores are normalized, e.g. by min-max normalization which transforms the scores to the interval $[0,1]$ or z-normalization which attains a zero mean and a unit variance of the score distribution. Normalization is necessary e.g. if different fingerprint matchers are combined or if different modalities (e.g. iris, face, fingerprint) are fused (refer to [74] and [103] for a survey). After normalization, score fusion can be performed. The basic combination rules are self-explaining:

- Sum rule

- Max rule

- Median rule

- Min rule

- Product rule

In [84], these rules are embedded into a common theoretical framework originating from Bayesian theory. In Chapter 2, we applied above listed rules for improving the verification performance in the case of matching adult fingerprints with unscaled and rescaled juvenile prints. Transformation-based score fusion is examined in the following sections.

Furthermore, image-level fusion and feature-level fusion are discussed in the context of multiple samples. E.g. in mosaicking [77, 127], two images are aligned and combined to one mosaicked image. Mosaicking improves the recognition rates in comparison to the individual images. The combination of different templates acquired from multiple impressions of the same finger are discussed under the headwords template consolidation and template adaptation. Consolidation refers to the enrollment phase, whereas adaption indicates modifications 
of the database template during later recognition attempts. Remembering the fingerprint growth analysis, we can imagine the case of a juvenile enrolling to a database, e.g. for access control to a library. Assuming that the person visits the library regularly, these recognition attempts give the system the opportunity to adapt to the changing, i.e. growing finger, and the template might be rescaled in this way without explicitly knowing about the grow process.

\subsection{Multi-Level Judgment Aggregation}

All aforementioned fusion techniques have their benefits and merits. And it is clear that in some scenarios only score-based, rank based or decision-based fusion is possible, because e.g. matchers are combined that act as black boxes and they output nothing but a score, rank or binary decision. Or another scenario is imaginable in which the matching algorithms are more transparent, cooperative and talkative, but they do not share mutual features, e.g. if a ridge-based algorithm is combined with a correlation-based and a Gabor feature-based matching algorithm. However, in most cases they do extract mutual features. A report on the Fingerprint Verification Competition 2004 states that 27 out of 29 participating algorithms extract minutiae as feature used for global or local matching [35]. For a moment, let us image what kind of minutiae template could be build, if these 27 algorithms cooperated and shared the pieces of information they gained from the image. Coinstantaneously the question arises: which strategy can be adopted for aggregating these extraction results?

The idea of judgment aggregation is to assemble as many pieces of information as possible on each level and render a judgment which is more substantiated and meaningful than the best of the individual judgments. The term 'judgment aggregation' is preferred in comparison to 'fusion' in order to indicate the capability of architecture to deal with incoherent and abstaining judgments [125] that goes beyond linear averaging. Let us imagine a board of judges or experts who shall deliver a judgment on a certain issue. Some of the judges may abstain from a decision, others might deliver incoherent judgments. [125] gives the example of experts forecasting probabilities. If expert Alice assigns a probability of 0.75 to event $X$ and a probability of 0.25 to the occurrence of $X \operatorname{or} Y$, she delivers incoherent judgments. Let us consider the level of orientation estimation. Here, an OF estimation method can be regarded as an expert and the orientation estimation for a certain pixel as a judgment. Method A may be unable to estimate the orientation for certain pixels (abstaining) and if estimations of neighboring pixels are discontinuous at locations other than the core and delta points, these judgments can be considered as incoherent. Furthermore, the judgment aggregation should account for the fact that experts have different skills. E.g. let us consider the scenario of combining three $\mathrm{OF}$ estimation methods named A, B and C. We assume that A and B are in general robust to noise, whereas $\mathrm{C}$ works only for regions of high quality and produces erroneous estimations for noisy parts of the image. Any kind of simple averaging of the three estimations will lead to an impaired OF estimation for low quality images. On the other hand, if the different skills are factored in, the weakness of method $\mathrm{C}$ can become a strength: if all methods agree on a judgment, i.e. if all estimations are located within a small cone, this allows not only to derive 
a combined estimation, but there is also reason to have confidence in the estimation and conclude that the pixel belongs probably to a good quality region. The conclusions may be even more specific, e.g. if it is know that a certain method is prone to fail especially in dry parts with broken ridge lines. The term 'multi-level' indicates that judgment aggregation should be performed on all possible levels, and in particular on the following levels (please regard this as an unordered list of partially overlapping, deeply interconnected tasks and bear in mind that different algorithms may operate in different sequences on different subsets):

- image quality estimation (global or local)

- segmentation

- orientation field estimation

- ridge frequency $(\mathrm{RF})$ estimation

- image enhancement

- binarization

- minutiae extraction and evaluation

- alignment

- score normalization

Subsequently, a few practical applications are presented which illustrate the potential of the design. On the OF level, a combination of the line sensor method and the gradients based method is tested. Pertaining to the binarization level, some experiments with three different binarization methods were conducted in which a new binary image was obtained by majority voting for each pixel. It is of interest to investigate this further. In Section 8.3, five image enhancement variants are described which are applied in Section 8.7 and 8.8 for judgment aggregation on the image enhancement and score normalization levels. In the next section, the general experimental setup is described and details of the used fingerprint image databases, verification protocols and performance measure are given.

\subsection{Test Setup}

The experiments were conducted on all available data sets from the series of the fingerprint verification competitions (FVC). Each competition held in the years 2000 [99], 2002 [100] and 2004 [101] comprises of four databases, and for each databases, 8 impressions of 110 fingers are provided. In all competitions, database 4 is made up of artificially generated fingerprints. The images of first 100 fingers were used in the competition, and the images of the other 10 fingers of each database were published in advance, so that the participants could tune their algorithms according to the sample. Table 8.1 gives an overview of the database properties. 


\begin{tabular}{|r|c|c|c|}
\hline Database & Sensor Type & $\begin{array}{c}\text { Image size } \\
\text { in pixels }\end{array}$ & $\begin{array}{c}\text { Resolution } \\
\text { in DPI }\end{array}$ \\
\hline \hline FVC2000 DB1 & Low-cost Optical Sensor & $300 \times 300$ & 500 \\
\hline DB2 & Low-cost Capacitive Sensor & $256 \times 364$ & 500 \\
\hline DB3 & Optical Sensor & $448 \times 478$ & 500 \\
\hline DB4 & Synthetic Generator & $240 \times 320$ & about 500 \\
\hline \hline FVC2002 DB1 & Optical Sensor & $388 \times 374$ & 500 \\
\hline DB2 & Optical Sensor & $296 \times 560$ & 569 \\
\hline DB3 & Capacitive Sensor & $300 \times 300$ & 500 \\
\hline DB4 & SFinGe v2.51 & $288 \times 384$ & about 500 \\
\hline \hline FVC2004 DB1 & Optical Sensor & $640 \times 480$ & 500 \\
\hline DB2 & Optical Sensor & $328 \times 364$ & 500 \\
\hline DB3 & Thermal sweeping Sensor & $300 \times 480$ & 512 \\
\hline DB4 & SFinGe v3.0 & $288 \times 384$ & about 500 \\
\hline
\end{tabular}

Table 8.1: Overview of the 12 FVC databases used in this thesis. Additional information can be found at the FVC websites: http://bias.csr.unibo.it/fvc200x/ where $\mathrm{x}$ has to be replaced by 0,2 or 4 .

In this thesis, tests are performed on all 12 databases and the enhancement is not adjusted to any specific database. The enhancement variants described in the next section are executed on all 9600 images with the same parameters.

The following verification protocol was established for the performance evaluation in FVC 2000 [99] and applied in all subsequent competitions:

Genuine Recognition Attempts For each of the 100 fingers, the first impression is matched against the seven other impressions, the second against the remaining six and so on, adding up to 28 genuine recognition attempts per finger and 2800 per database.

Impostor Recognition Attempts For the impostor recognition attempts, only the first of the eight available impressions is used. The first print of the first finger is matched against the first prints of 99 other fingers, the first print of the second finger is matched against the remaining 98 first prints of the other fingers and so on. This adds up to 4950 impostor recognition attempts per database.

All subsequently presented results are obtained following exactly the above described verification protocol. False acceptance rates (FARs), false rejection rates (FRRs) and the equal error rates (EERs) are calculated as explained in [99]. FARs are also called 'false match rates' (FMRs) and FRRs are also known as 'false non-match rates' (FNMRs). In this thesis, the EER is used as the main performance indicator, because it is particularly suitable for measuring the reduction of false accepts and false rejects by image enhancement. Other performance indicators like FMR100, FMR1000 and ZeroFMR [18] are very relevant for the evaluation of practical applications [71], but here, they are only of subordinate interest, and for reasons of clarity and readability, they are omitted. 
On comparing different image enhancement and fusion approaches, it is very important to account for the applied verification protocol. The relation of genuine to impostor recognition attempts as well as the choice of certain subsets of images from a database massively influences the performance of the system and results become incomparable. Therefore, the conduction of all experimentations according to the full FVC protocol is of great significance in order to produce results that can be compared with other methods.

For minutiae extraction and matching, the same software was applied as described in Section 2.4.2.

\subsection{Image Enhancement by Applying the Curved Gabor Filter}

In this section, five image enhancement versions are described which apply curved regions for the ridge frequency estimation as introduced in Section 6.2 and the curved Gabor filter (CGF) introduced in Section 7.3. Acronyms are established, so that they can referenced in all subsequent sections. Due to time constraints and computational limitations, these enhancement variants are sample applications with an ad hoc parameter choice. Further parameter combinations should be tested more systematically in order to investigate possible improvements by better parameter choices.

All enhancement versions have the same general layout: first, the $\mathrm{OF}$ is estimated, either by a single method or by a combination of methods. Second, a raw, pixelwise RF image is obtained using the curved regions based RF estimation and the OF from the first step. Afterwards, the RF is smoothed by averaging in a window. Finally, the curved Gabor filter is applied. Input arguments are the $\mathrm{OF}$, the smoothed $\mathrm{RF}$ and the tuning parameters $\sigma_{x}$ and $\sigma_{y}$. Segmentation or quality estimation is not explicitly performed in this experimentation. Since image enhancement is only performed for those pixels that are equipped with orientation and ridge frequency estimation, all other pixels are set to the global mean gray value and regarded as background.

Enhancement version E1. Two orientation fields are computed applying the line sensor method and the gradients based method with a smoothing window size of 33 pixels. The OFs are compared at each pixel. If the angle between both estimations is smaller than $15^{\circ}$, the orientation of the combined $\mathrm{OF}$ is set to the average of the two. Otherwise, the pixel is marked as missing. Afterwards, all inner gaps are reconstructed as described in Section 4.4.2 and up to a radius of 16 pixels, the orientation of the outer proximity is extrapolated. The raw $\mathrm{RF}$ image is computed and smoothed in a window of size 49 pixels. Finally, the curved Gabor filter is applied with $\sigma_{x}=4$ and $\sigma_{y}=4$.

Enhancement version E2. Like in the previous version, two orientation fields are estimated using the line sensor method and the gradients based method (smoothing window size of 33 pixels). Here, the condition for averaging is an 
angle smaller than $10^{\circ}$ between both estimations and pixels for which both methods disagree (i.e. forming an angle $\geq 10^{\circ}$ ) are not reconstructed. Since image enhancement is performed only for pixels endowed with an orientation estimation, this leads to gaps in the enhanced image which are filled with the mean gray value. The raw RF image is smoothed over windows of 33 pixels width and the CGF parameters are $\sigma_{x}=5$ and $\sigma_{y}=4$.

Enhancement version E3. The OF is estimated by the line sensor method. A window size of 33 pixels is used for smoothing the raw RF image and the curved Gabor filter parameters are $\sigma_{x}=6$ and $\sigma_{y}=7$.

Enhancement version E4. Here, the line sensor method is applied with a grid size of 6 pixels (instead of 8 pixels which is used in all cases when the grid size is not explicitly stated). The averaging window for RF smoothing is 49 pixels wide and $\sigma_{x}=6, \sigma_{y}=7$ are the tuning parameters of the CGF.

Enhancement version E5. For version E5, the OF is estimated using the gradients based method with an averaging window of size 33 pixels. The ridge frequency is smoothed over windows of 65 pixels and the curved Gabor filter is applied with $\sigma_{x}=4$ and $\sigma_{y}=4$.

\subsubsection{Results and Discussion}

Table 8.2 specifies the EERs which are obtained, if the the three algorithms BZ3, VFM and VFG (see Section 2.4.2) are applied to the original images and enhanced images using version E1. On comparing the results, it is important to bear in mind that the free NIST software binarizes the grayscale input image and extracts the minutiae without performing any image enhancement, whereas the two algorithms derived from the Neurotechnology VeriFinger 5.0 SDK, VFM and VFG, have a built-in image enhancement which can not be turned off. In the case of these two algorithms, the test should be understood as a comparison of the VeriFinger built-in image enhancement versus a pre-enhancement using version E1 as described in Section 8.3 followed by the VeriFinger built-in enhancement. Bearing in mind that the VeriFinger software belonged to the top five algorithms in the FVC competitions, it is quite astonishing that EER reductions of up to $75 \%$ are possible by the additional pre-enhancement using version E1. On the other hand, the results on FVC 2002 database 1 remind us of the risk that image enhancement can also impair the performance.

Basically, there are two types of errors which can occur during the minutiae extraction stage: first, true minutiae can be missed and second, false minutiae can be extracted. The image enhancement techniques we regard in this thesis aim at reducing the number of extraction errors. Ideally, fewer true minutiae are missed after the enhancement without introducing additional false minutiae. The image enhancement version E1 is well suited to function as a stand-alone image enhancement step, whereas e.g. version E2 is specifically designed for enhancing only those parts of the image which can be regard as reliable in terms of agreeing orientation estimations by the line sensor and gradients based method. 


\begin{tabular}{|c|c|c|c|}
\hline Matcher: BZ3 & Original images & Enhancement E1 & EER Change \\
\hline$\overline{\text { FVC2000 DB1 }}$ & $25.96 \%$ & $4.18 \%$ & $-83.9 \%$ \\
\hline DB2 & $4.8 \%$ & $2.68 \%$ & $-44.2 \%$ \\
\hline DB3 & $6.3 \%$ & $4.93 \%$ & $-21.7 \%$ \\
\hline DB4 & $10.41 \%$ & $4.85 \%$ & $-53.4 \%$ \\
\hline FVC2002 DB1 & $4.13 \%$ & $4.2 \%$ & $+1.7 \%$ \\
\hline DB2 & $3.32 \%$ & $2.38 \%$ & $-28.3 \%$ \\
\hline DB3 & $8.54 \%$ & $9.13 \%$ & $+6.9 \%$ \\
\hline DB4 & $6.92 \%$ & $4.41 \%$ & $-36.3 \%$ \\
\hline 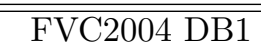 & $\overline{15.06 \%}$ & $11.42 \%$ & $-24.2 \%$ \\
\hline DB2 & $16.34 \%$ & $5.93 \%$ & $-63.7 \%$ \\
\hline$\overline{\text { DB3 }}$ & $7.37 \%$ & $5.31 \%$ & $-28 \%$ \\
\hline DB4 & $9.88 \%$ & $6.8 \%$ & $-31.2 \%$ \\
\hline Matcher: VFM & Original images & Enhancement E1 & EER Change \\
\hline FVC2000 DB1 & $4.5 \%$ & $1.65 \%$ & $-63.3 \%$ \\
\hline DB2 & $1.05 \%$ & $0.74 \%$ & $-29.5 \%$ \\
\hline$\overline{\mathrm{DB}} 3$ & $8.04 \%$ & $4.61 \%$ & $-42.7 \%$ \\
\hline DB4 & $3.77 \%$ & $2.6 \%$ & $-31 \%$ \\
\hline$\overline{\text { FVC2002 DB1 }}$ & $\overline{0.76 \%}$ & $\overline{0.95 \%}$ & $+25 \%$ \\
\hline DB2 & $1.08 \%$ & $0.46 \%$ & $-57.4 \%$ \\
\hline DB3 & $2.9 \%$ & $2.31 \%$ & $-20.3 \%$ \\
\hline DB4 & $2.09 \%$ & $1.23 \%$ & $-41.1 \%$ \\
\hline FVC2004 DB1 & $8.29 \%$ & $6.87 \%$ & - $-17.1 \%$ \\
\hline DB2 & $7.1 \%$ & $4.18 \%$ & $-41.1 \%$ \\
\hline DB3 & $7.35 \%$ & $4.37 \%$ & $-40.5 \%$ \\
\hline DB4 & $2.38 \%$ & $2.29 \%$ & $-3.8 \%$ \\
\hline " Matcher: VFG & Original images & Enhancement E1 & EER Change \\
\hline FVC2000 DB1 & $4.37 \%$ & $1.03 \%$ & - $76.4 \%$ \\
\hline DB2 & $0.87 \%$ & $0.5 \%$ & $-42.5 \%$ \\
\hline DB3 & $6.7 \%$ & $3.19 \%$ & $-52.4 \%$ \\
\hline DB4 & $3.64 \%$ & $1.45 \%$ & $-60.2 \%$ \\
\hline FVC2002 DB1 & $0.68 \%$ & $0.74 \%$ & + \\
\hline$\overline{\text { DB2 }}$ & $0.9 \%$ & $0.39 \%$ & $-56.7 \%$ \\
\hline$\overline{\mathrm{DB}} 3$ & $2.32 \%$ & $1.39 \%$ & $-40.1 \%$ \\
\hline DB4 & $1.67 \%$ & $0.44 \%$ & $-73.7 \%$ \\
\hline FVC2004 DB1 & $8.78 \%$ & $6.16 \%$ & $-29.8 \%$ \\
\hline DB2 & $6.62 \%$ & $2.93 \%$ & $-63.9 \%$ \\
\hline DB3 & $5.32 \%$ & $2.3 \%$ & $-56.8 \%$ \\
\hline DB4 & $1.97 \%$ & $1.31 \%$ & $-33.5 \%$ \\
\hline
\end{tabular}

Table 8.2: EERs for the original and enhanced images. For each entry, a full FVC verification test with 7750 recognition attempts was conducted. BZ3, VFM and VFG denote the three algorithms described in Section 2.4.2. 
E2 targets for improving the clarity of the ridge structure in these parts, so that further true minutiae can be discovered without adding false minutiae. On the downside, a substantial amount of information is lost, because the regions in which the $\mathrm{OF}$ estimations disagree, i.e. forming an angle $\geq 10^{\circ}$, are ignored and set to the mean gray value. For these reasons, E2 is unsuitable as a standalone enhancement method. An obvious concept, however, is the combination of the minutiae from the original and enhanced image following the idea that additional true minutiae are discovered in the foreground of the enhanced image and that a combined template will perform better than each of the individual templates. This notion is examined in the next section.

\subsection{Score Fusion vs. Minutiae Fusion}

In this section, we investigate whether the EERs can be further reduced by two traditional fusion approaches: score-level fusion and minutiae-level fusion.

On the score level, the VFM score for a recognition attempt with the original images is combined with the VFM score obtained on the enhanced images for each of the five enhancement variants. Ideally, the enhancement amplifies the scores of the genuine recognition attempts without changing the scores of the impostor recognition attempts. In some cases, matching two original images of the same finger produces scores close to 0, especially for low quality images, whereas matching the enhanced images may lead to scores which lie above the threshold at the EER. Averaging the scores (which is equivalent to applying the sum rule) would not be favorable for these cases. Therefore, scores are fused by the max rule [84] in this test.

Two minutiae templates are combined by simply attaching all minutiae of one template to the other with one constraint: in avoidance of duplicates, existing minutiae are checked whether they are located in a radius of 5 pixels and they have a similar direction $\left(\leq 15^{\circ}\right)$. If such a minutia is already part of the template, than the second, similar minutia is not added.

Regarding the results listed in Table 8.3, a number of conclusions can be drawn. Generally, both score-level fusion and minutiae level fusion have the potential to improve the verification performance, partially EERs shrink down to a quarter of the original rate. On the other hand, in a few cases the EERs increase, especially for the combination of algorithm E3 and minutiae fusion. Relating to the order of magnitude, both fusion techniques yield approximately equivalent results. Performance differences are apparent between the enhancement variants. Score and minutiae fusion involving version E1 which combines the line sensor and gradients based method for OF estimations achieves the lowest EER on 11 out of the 12 databases. Additional to the combinations listed in Table 8.3, minutiae and score fusion of two or three enhancement versions (excluding the scores and templates of the original images) were also tested. These combinations performed similar to those which fuse E2 to E5 in Table 8.3, but EERs were higher than the EERs achieved by the combination of E1 and the 


\begin{tabular}{|l|c|c|c|c|}
\hline FVC 2000 & Database 1 & Database 2 & Database 3 & Database 4 \\
\hline \hline Original images & & & & \\
\hline \hline Score: O. and E1 & $1.5 \%$ & $1.05 \%$ & $8.04 \%$ & $3.77 \%$ \\
\hline Minutiae: O. and E1 & $1.36 \%$ & $0.7 \%$ & $4.39 \%$ & $1.64 \%$ \\
\hline Score: O. and E2 & $1.53 \%$ & $0.79 \%$ & $4.25 \%$ & $1.58 \%$ \\
\hline Minutiae: O. and E2 & $1.36 \%$ & $0.94 \%$ & $4.59 \%$ & $2.55 \%$ \\
\hline Score: O. and E3 & $1.88 \%$ & $0.77 \%$ & $5.29 \%$ & $2.4 \%$ \\
\hline Minutiae: O. and E3 & $1.81 \%$ & $0.98 \%$ & $5.49 \%$ & $3.28 \%$ \\
\hline Score: O. and E4 & $1.79 \%$ & $0.9 \%$ & $5.44 \%$ & $2.16 \%$ \\
\hline Minutiae: O. and E4 & $1.46 \%$ & $0.91 \%$ & $5.06 \%$ & $2.16 \%$ \\
\hline Score: O. and E5 & $1.45 \%$ & $0.79 \%$ & $5.04 \%$ & $2.08 \%$ \\
\hline Minutiae: O. and E5 & $1.43 \%$ & $0.91 \%$ & $5.27 \%$ & $1.7 \%$ \\
\hline \hline & & & & \\
FVC 2002 & Database 1 & Database 2 & Database 3 & Database 4 \\
\hline \hline Original images & $0.76 \%$ & $1.08 \%$ & $2.9 \%$ & $2.09 \%$ \\
\hline \hline Score: O. and E1 & $0.67 \%$ & $0.28 \%$ & $1.25 \%$ & $0.8 \%$ \\
\hline Minutiae: O. and E1 & $0.72 \%$ & $0.38 \%$ & $1.56 \%$ & $0.9 \%$ \\
\hline Score: O. and E2 & $0.67 \%$ & $0.43 \%$ & $2.08 \%$ & $1.17 \%$ \\
\hline Minutiae: O. and E2 & $0.62 \%$ & $0.42 \%$ & $2.07 \%$ & $1.24 \%$ \\
\hline Score: O. and E3 & $0.82 \%$ & $0.31 \%$ & $2.12 \%$ & $1.75 \%$ \\
\hline Minutiae: O. and E3 & $0.87 \%$ & $0.41 \%$ & $2.41 \%$ & $2.31 \%$ \\
\hline Score: O. and E4 & $0.75 \%$ & $0.39 \%$ & $2.0 \%$ & $1.71 \%$ \\
\hline Minutiae: O. and E4 & $0.75 \%$ & $0.46 \%$ & $1.88 \%$ & $1.54 \%$ \\
\hline Score: O. and E5 & $0.69 \%$ & $0.49 \%$ & $2.18 \%$ & $1.33 \%$ \\
\hline Minutiae: O. and E5 & $0.81 \%$ & $0.7 \%$ & $2.45 \%$ & $0.95 \%$ \\
\hline \hline & & & & \\
FVC 2004 & Database 1 & Database 2 & Database 3 & Database 4 \\
\hline \hline Original images & $8.29 \%$ & $7.1 \%$ & $7.35 \%$ & $2.38 \%$ \\
\hline \hline Score: O. and E1 & $5.84 \%$ & $4.12 \%$ & $3.95 \%$ & $1.5 \%$ \\
\hline Minutiae: O. and E1 & $6.48 \%$ & $4.01 \%$ & $4.99 \%$ & $1.88 \%$ \\
\hline Score: O. and E2 & $6.67 \%$ & $4.43 \%$ & $4.5 \%$ & $2.17 \%$ \\
\hline Minutiae: O. and E2 & $7.05 \%$ & $4.57 \%$ & $4.7 \%$ & $2.21 \%$ \\
\hline Score: O. and E3 & $7.35 \%$ & $5.07 \%$ & $4.74 \%$ & $2.15 \%$ \\
\hline Minutiae: O. and E3 & $7.67 \%$ & $5.33 \%$ & $5.33 \%$ & $3.07 \%$ \\
\hline Score: O. and E4 & $6.46 \%$ & $4.64 \%$ & $4.86 \%$ & $2.05 \%$ \\
\hline Minutiae: O. and E4 & $6.49 \%$ & $4.22 \%$ & $5.06 \%$ & $2.56 \%$ \\
\hline Score: O. and E5 & $6.2 \%$ & $5.42 \%$ & $5.08 \%$ & $1.55 \%$ \\
\hline Minutiae: O. and E5 & $6.8 \%$ & $4.55 \%$ & $4.95 \%$ & $1.69 \%$ \\
\hline
\end{tabular}

Table 8.3: EERs for verification tests applying algorithm VFM. Performance on the original images is compared with score fusion using the max rule and combination of minutiae templates. O. indicates the original images.. 
original images. Interestingly, E1 as a standalone method performs better than the score or minutiae fusion of $\mathrm{E} 1$ and the original images on three databases of FVC 2004. Bearing the in mind that FVC 2004 contains many low quality images, it suggests itself to incorporate quality estimation into decisions at the score level.

\subsection{Image Quality Based Score Normalization}

Fingerprint image quality influences the verification performance. In low quality images, large parts of the image or the whole image is disturbed by one or more kinds noise. As discussed previously, typical types of noise are caused by wetness or dryness of the finger, or by scars. During one the scanning sessions for acquiring the fingerprints used in FVC 2004, the fingers of the volunteers were intentionally dried and moistened in order to introduce this kind of difficulty. Generally, dryness interrupts the ridges, wetness causes neighboring ridges to conglutinate and scars usually cut some ridges along a line, often simultaneously joining the cut neighboring lines near the cutting point. During the minutia extraction, it is more likely that true minutiae are missed and false minutiae are discovered in regions disturbed by noise.

Verification errors can be reduced, if the image quality is taken into account during the score fusion [37]. This approach is also useful for multibiometric fusion (e.g. see [116] for fusion of fingerprint and iris).

For our study of possible improvements by an image quality based score normalization, we apply the NFIQ (NIST fingerprint image quality) software [139]. NFIQ outputs a discrete number in the range from 1 to 5 as a global quality estimation for the input image. 1 indicates excellent quality and 5 stands for poor quality. Quality assessment is viewed as a classification problem by NFIQ. The decisive weights were determined using a neural network at the NIST and their training images may have varied perspicuously from the FVC images. This is how I interpret the fact that my human quality estimation disagrees with the NFIQ assessment on many of the visually inspected images. Another point of critique is the susceptibility of the NFIQ quality estimation to slight changes. In my experimentation, I tested NFIQ on the original images and on various smoothed variants using median or Gaussian filters [138] and found assessment jumps from 1 to 5 for slightly smoothed images which visually appeared to have the about same quality. In principle, it is desirable for an image quality estimation method to be robust to small changes of the image.

\subsubsection{Test Details}

The basic idea of quality based score normalization is to compensate for poor quality by upgrading the scores obtained on matching low quality fingerprint images. In our experimentation, boosting low quality images was performed in the following way: for each of the 7750 recognition attempts per FVC database, the max value (lowest quality) of the quality assessment by NFIQ for the two involved images is determined. The scores of recognition attempts with qualities between 2 and 5 are valorized by factors $q_{2}$ to $q_{5}$. Combinations are tested with 


\begin{tabular}{|l|c|c|c|c|}
\hline FVC 2000 & Database 1 & Database 2 & Database 3 & Database 4 \\
& & & & \\
\hline \hline Original images & $4.5 \%$ & $1.05 \%$ & $8.04 \%$ & $3.77 \%$ \\
\hline Q(Original images) & $4.28 \%$ & $0.92 \%$ & $6.91 \%$ & $3.58 \%$ \\
\hline \hline Enhancement E1 & $1.65 \%$ & $0.74 \%$ & $4.61 \%$ & $2.6 \%$ \\
\hline Q(Enhancement E1) & $1.59 \%$ & $0.6 \%$ & $3.48 \%$ & $2.39 \%$ \\
\hline \hline SF(O. and E1) & $1.02 \%$ & $0.7 \%$ & $4.39 \%$ & $1.64 \%$ \\
\hline Q(SF(O. and E1) $)$ & $1.00 \%$ & $0.52 \%$ & $3.35 \%$ & $1.57 \%$ \\
\hline \hline & & & & \\
FVC 2002 & Database 1 & Database 2 & Database 3 & Database 4 \\
\hline \hline Original images & $0.76 \%$ & $1.08 \%$ & $2.9 \%$ & $2.09 \%$ \\
\hline Q(Original images $)$ & $0.74 \%$ & $0.94 \%$ & $2.51 \%$ & $2.03 \%$ \\
\hline \hline Enhancement E1 & $0.95 \%$ & $0.46 \%$ & $2.31 \%$ & $1.23 \%$ \\
\hline Q(Enhancement E1) & $0.86 \%$ & $0.4 \%$ & $2.02 \%$ & $1.1 \%$ \\
\hline \hline Score: O. and E1 & $0.67 \%$ & $0.28 \%$ & $1.25 \%$ & $0.8 \%$ \\
\hline Q(SF(O. and E1) $)$ & $0.63 \%$ & $0.23 \%$ & $1.15 \%$ & $0.75 \%$ \\
\hline \hline & & & & \\
FVC 2004 & Database 1 & Database 2 & Database 3 & Database 4 \\
\hline \hline Original images & $8.29 \%$ & $7.1 \%$ & $7.35 \%$ & $2.38 \%$ \\
\hline Q(Original images) & $7.67 \%$ & $5.72 \%$ & $6.24 \%$ & $2.38 \%$ \\
\hline \hline Enhancement E1 & $6.87 \%$ & $4.18 \%$ & $4.37 \%$ & $2.29 \%$ \\
\hline Q(Enhancement E1) & $6.44 \%$ & $3.47 \%$ & $3.02 \%$ & $2.17 \%$ \\
\hline \hline Score: O. and E1 & $5.84 \%$ & $4.12 \%$ & $3.95 \%$ & $1.5 \%$ \\
\hline Q(SF(O. and E1) $)$ & $5.53 \%$ & $3.21 \%$ & $2.67 \%$ & $1.4 \%$ \\
\hline
\end{tabular}

Table 8.4: EERs of verification tests using algorithm VFM. O denotes the original images, SF score fusion and Q indicates that quality based score normalization was performed.

the constraints $1 \leq q_{5} \leq 5$ and $q_{2} \leq q_{3} \leq q_{4} \leq q_{5}$. Steps of 0.1 were considered. E.g. in Table 8.4, the algorithm VFM achieved an EER of $7.1 \%$ on matching the templates of the original images of FVC 2004 database 2. If matching scores are multiplied by $q_{5}=2.8, q_{4}=1.7, q_{3}=1.5, q_{2}=1.1$, the EER is reduced to $5.72 \%$.

\subsubsection{Results and Discussion}

Pertaining to the results listed in Table 8.4, it can be positively noted that in all 36 cases the EERs of the verification tests applying the quality based normalized scores are smaller (or equal in one case) than the EERs of the tests using the corresponding non-normalized scores. This is far from being self-evident, especially bearing in mind that NFIQ produced a number of erroneous estima- 
tions for the inspected images. However, in most cases the gained improvement was very small. It would be of interest to test other quality estimation methods than NFIQ, e.g. [91], and also, to compare it to quality estimations by a human expert. In my opinion, it is quite obvious that on the one hand, some further reduction of the EERs is possible by applying better quality estimation methods, but on the other hand, there are limits which can not be overcome by regarding image quality only. In Section 8.8 , image quality will be an important part of our considerations in conjunction with other factors.

\subsection{The Doddington Zoo Effect}

The 'Doddington zoo effect' is named after George Doddington who investigated the performance variability in speaker recognition systems [32]. Based on the observation that recognition errors are inhomogeneously distributed among speakers, population statistics are analyzed in order to detect speakers who perform conspicuously well or poor, because these speakers strongly influence the performance of whole the system. Afterwards, this concept was transferred from speaker recognition to other biometric modalities including fingerprint recognition. This approach became widely known in the biometrics community and received a lot of attention recently. The objective of this section is to examine whether the Doddington zoo effect can be capitalized for a user-specific score adaptation in order to reduce the number of verification errors on the FVC databases used in our experimentation.

Depending on the performance characteristics, users are assigned to following classes which have been given animal names:

- Sheep: Users who generally perform well. They achieve high scores in genuine recognition attempts and low scores in impostor recognition attempts. These users are ideal from a biometric system's point of view.

- Goats: Users who are particularly difficult to match. They achieve low scores when matched against themselves or others and therefore, they tend to cause false rejects.

- Lambs: Users who are vulnerable to impersonation. They achieve high scores when being matched against resulting in false accepts.

- Wolves: Users who are disproportionately likely to be successful as an impostor and in doing so, they tend to produce false accepts.

For our analysis, lambs and wolves are regarded conjointly, because we do not consider the sequence in which images or templates are matched and both tend to cause false accepts. An expansion of biometric menagerie by four additional animals is proposed in $[150,151]$.

The idea of exploiting the Doddington zoo effect is to identify users belonging to the classes goat or lamb/wolf, and then magnify (for goats) or diminish (for lamb/wolf) the matching scores, so that ideally all scores of the genuine recognition attempts for that user are located above the threshold and for impostor scores end up below the threshold. Ways of exploiting the effect can be 


\begin{tabular}{|l|c|c|c|c|}
\hline FVC 2004 & DB 1 & DB 2 & DB 3 & DB 4 \\
\hline \hline Error free & 0 & 6 & 7 & 26 \\
\hline \hline Involved in false accepts (FA) & 49 & 57 & 58 & 55 \\
\hline Involved in false rejects (FR) & 1 & 2 & 7 & 4 \\
\hline Involved in FAs and FRs & 50 & 25 & 28 & 15 \\
\hline \hline Separable & 40 & 62 & 49 & 49 \\
\hline Inseparable & 60 & 32 & 44 & 25 \\
\hline
\end{tabular}

Table 8.5: Based on the verification results achieved by score fusion of the original images and E1 using VFM (see Table 8.3), fingers are divided into four classes depending on the kind of verification errors they are involved in. Separable indicates that the minimum genuine score for a finger is greater than the maximum impostor, so that in the best case, a user-specific score normalization could transform that finger from one of the three error categories into the class 'error free'. Inseparable denotes that the minimum genuine score for a finger is less or equal to the maximum impostor score.

found in [32] and [131]. The latter describes its application for fusion of iris and fingerprint data. Two very similar methods are F-ratio [122] and Z-shift [123]. Fingerprint pattern classification was analyzed in [146] and the author comes to the conclusion that wolves and lambs exist in fingerprint recognition (with gradual transition between sheep and these populations). In [16], verification performance after single enrollment was statistically analyzed using a commercial matcher. $10 \%$ of the users were more likely to be involved in false rejects than the remaining $90 \%$. Based on this result, the authors attest these $10 \%$ a 'goat'-like behavior. Goats are also in main focus of [58]. Based on studying 6000 subjects who are frequent users of US-VISIT, the authors reject the notion of goats and summarize:

There have been a variety of statements made in the recent past that a small percentage of people (usually $2 \%$ ) cannot be fingerprinted due to poor quality fingers. This study shows these statements are based on misconceptions: the fact that some small percentage of fingerprints may be hard to match does not mean that a corresponding percentage of people are hard to match.

Scrutinizing the false rejects which remain after image enhancement, I agree with the statement. There are no goats in the FVC databases, i.e. no persons who are consistently hard to match. Rather, the false accepts are caused by single impressions which have a particularly low quality or a small area of overlap with the other impressions of that finger (a more detailed illumination of this fact follows in Sections 8.8; e.g. see Figure 8.2).

\subsubsection{Discussion}

Taking into account the Doddington zoo effect certainly has its merits, especially in speech and speaker recognition. And it can be useful for establishing 
criteria to verify the adequacy of a template at enrollment and the choice of selection criteria for multimodal fusion [124] In fingerprint recognition, analyzing the verification performance of a system for the Doddington zoo effect can be a very useful approach for detecting the weaknesses of system. E.g. if persons whose fingers are disturbed by scars show a goat-like behavior, this fact could indicate that the algorithm has a flaw for this kind of noise. On the other hand, if user-specific score normalization is applied in order not minimize verification errors, a drawback is that impostor scores are required as training data [36], i.e. for computing the z-norm. In real life scenarios, fulfilling this prerequisite might not be feasible.

Table 8.5 illustrates the potential and the limitations of what be achieved by a user-specific score normalization. There is a large number of separable fingers which means that their minimum genuine score is greater than the maximum impostor score, so that in principle, a user-specific score normalization could transform in such a way that after the transformation, all genuine scores are located above the score threshold at the EER and all impostor scores below. On the other hand, the inseparable cases show the limitations. No matter what transformation is applied, verification errors remain. In the light of the inseparable cases, it is unsatisfying that there is no hope to solve the pattern recognition challenge by applying a user-specific score normalization. That is way I decided not to pursue this direction. In Section 8.8, we will consider a novel approach for addressing this challenge.

\subsection{Cross Matching}

Score-level fusion is typically performed in a scenario with multiple algorithms acting as black boxes. Each algorithm outputs only a score which measures the similarity between the two fingerprints that are compared in the recognition attempt.

In our study, we experiment with different image enhancement variants which enables us to combine them with each other. In my opinion, this is a quite natural idea that has, to the best of my knowledge, not yet been considered in literature. The name cross matching was chosen, because each variant is combined with all other variants. Here, 'variant' shall include the original image as well as all enhanced versions. Templates can be cross-matched against other templates (BZ3, VFM), or images can be cross-matched against other images (VFG). The rationale behind this approach is that different enhancement versions may produce better or worse results on different images. E.g. one version may have very strong smoothing and ridge reconnecting properties and e.g. it may lead to the best results on dry images, whereas another enhancement version may achieve the best results on another type of noise. If e.g. in a genuine recognition attempt, an image with many scars is matched against a dry fingerprint, the highest score may be obtained by a combination of two different enhancement versions. 


\begin{tabular}{|c|c|c|c|c|}
\hline FVC 2000 & Database 1 & Database 2 & Database 3 & Database 4 \\
\hline Original images & $4.5 \%$ & $1.05 \%$ & $8.04 \%$ & $3.77 \%$ \\
\hline $\begin{array}{l}\text { Trad. score fusion of } \\
\text { O and E1 }\end{array}$ & $1.02 \%$ & $0.7 \%$ & $4.39 \%$ & $1.64 \%$ \\
\hline $\begin{array}{c}\text { Cross matching of } \\
\mathrm{O} \text { and E1 }\end{array}$ & $1.08 \%$ & $0.66 \%$ & $3.69 \%$ & $1.63 \%$ \\
\hline $\begin{array}{c}\text { Trad. score fusion of } \\
\text { O, E1 and E5 }\end{array}$ & $1.01 \%$ & $0.66 \%$ & $4.21 \%$ & $1.55 \%$ \\
\hline $\begin{array}{c}\text { Cross matching of } \\
\text { O, E1 and E5 }\end{array}$ & $0.96 \%$ & $0.62 \%$ & $3.33 \%$ & $1.34 \%$ \\
\hline FVC 2002 & Database 1 & Database 2 & Database 3 & Database 4 \\
\hline Original images & $0.76 \%$ & $1.08 \%$ & $2.9 \%$ & $2.09 \%$ \\
\hline $\begin{array}{l}\text { Trad. score fusion of } \\
\text { O and E1 }\end{array}$ & $0.67 \%$ & $0.28 \%$ & $1.25 \%$ & $0.8 \%$ \\
\hline $\begin{array}{c}\text { Cross matching of } \\
\mathrm{O} \text { and E1 }\end{array}$ & $0.72 \%$ & $0.25 \%$ & $1.23 \%$ & $0.79 \%$ \\
\hline $\begin{array}{l}\text { Trad. score fusion of } \\
\text { O, E1 and E5 }\end{array}$ & $0.66 \%$ & $0.28 \%$ & $1.25 \%$ & $0.78 \%$ \\
\hline $\begin{array}{c}\text { Cross matching of } \\
\text { O, E1 and E5 }\end{array}$ & $0.64 \%$ & $0.25 \%$ & $1.15 \%$ & $0.71 \%$ \\
\hline FVC 2004 & Database 1 & Database 2 & Database 3 & Database 4 \\
\hline Original images & $8.29 \%$ & $7.1 \%$ & $7.35 \%$ & $2.38 \%$ \\
\hline $\begin{array}{l}\text { Trad. score fusion of } \\
\text { O and E1 }\end{array}$ & $5.84 \%$ & $4.12 \%$ & $3.95 \%$ & $1.5 \%$ \\
\hline $\begin{array}{l}\text { Cross matching of } \\
\mathrm{O} \text { and E1 }\end{array}$ & $5.32 \%$ & $3.84 \%$ & $3.37 \%$ & $1.33 \%$ \\
\hline $\begin{array}{l}\text { Trad. score fusion of } \\
\text { O, E1 and E5 }\end{array}$ & $5.71 \%$ & $4.09 \%$ & $3.61 \%$ & $1.3 \%$ \\
\hline $\begin{array}{l}\text { Cross matching of } \\
\text { O, E1 and E5 }\end{array}$ & $4.96 \%$ & $3.27 \%$ & $3.0 \%$ & $1.1 \%$ \\
\hline
\end{tabular}

Table 8.6: Comparison of EERs for verification tests applying algorithm VFM. Performance of the traditional score fusion applying the max rule is compared to the verification performance of cross matching. O denotes the templates created on the original images. For enhancement versions E1 and E5, confer to Section 8.3 . 
In Table 8.6, the verification performance of traditional score fusion and cross matching is compared. Improvements are achieved by all three algorithms on the difficult databases (FVC 2000 DB 3 and all databases of FVC 2004). The best results are obtained by VFG. For reasons of comparability, Table 8.6 reports the results for algorithm VFM which is applied in previous and subsequent sections.

Another aspect is by far more important than the achieved improvements. Cross matching prepares score revaluation which is presented in the next section by potentially creating alternative matches in regard of the matched minutiae and the respective alignment. On comparing the available templates for each impression pairwise, it is obvious that they may contain different subsets of true minutiae and different sets of false minutiae. From this circumstance arises the possibility that different sets of minutiae are paired and regarded as best match by an algorithm. Recognition attempts are retroactively re-evaluated in the following section. Wrong alignments (non-fitting with respect to an introduced measure) are downgraded which can equally affect impostors and genuine users. If a genuine recognition attempt is devaluated due to non-fitting alignment, it is imperative to have alternative combinations in order to recognize the genuine user. Cross matching assists to provide these alternatives.

Once the currently existing obstacles at the matcher level are eliminated and additionally, local quality estimation methods are part of the architecture, then the main purpose of cross matching will be the integration of different methods on the image enhancement level, e.g. combining curved Gabor filters with anisotropic diffusion techniques and pyramid-based image enhancement.

\subsection{Score Revaluation}

Previously, we considered possible improvements by a quality based score normalization and by a user-dependent score adaption. Both approaches can reduce the EERs to some extent, but they also have their limitations. Let us remember our goal stated in the introduction of this thesis: assuming the individuality of fingerprints, how can a sufficient amount of discriminative information be extracted, so that for each recognition attempt a non-ambiguous decision between genuine users and impostors can be made?

Reckoning visualizations of false accepts and false rejects, it is quite obvious for a human being where the machine algorithm erred. Analogous to the beginnings of AFIS in the 1960s when algorithms learned from the way forensic experts process fingerprint images, the basic idea of score revaluation is to the teach the algorithm comparable skills for the post-matching stage.

The orientation field is a pivotal point in this approach. E.g. if a human expert glances at the recognition attempt depicted in Figure 8.1 and regards the proposed alignment and matched minutiae, it is instantaneously clear that these two images belong to two different fingers. Subsequently, we develop features and criteria allowing for an automated score revaluation. 


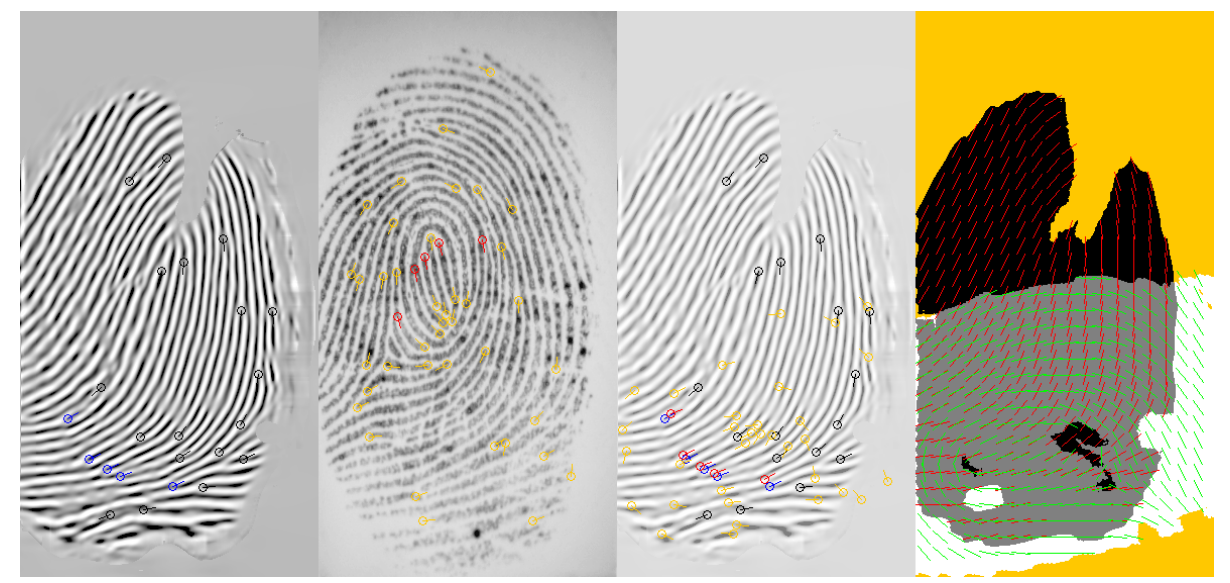

Figure 8.1: The impostor recognition attempt (f.l.t.r: finger 29 and 77 of FVC 2002 database 2 ) with the highest matching score (48, VFM, maximum of cross matching original images and enhancement version E1) before score revaluation. On the left, the enhanced image applying E1 for finger 29, original image for finger 77 aside. Matching minutiae are depicted in blue and red, non-matching in black and yellow. The third image from the left displays minutiae positions after alignment and the fourth the OFs after alignment. Black area indicates $\mathrm{OF}$ foreground for the first image and white OF foreground for the second image. Overlapping foreground is drawn in gray, background in orange. The OF belonging to the first images is visualized in red, the OF for the second in green. Obviously, the OFs do not fit, if the second OF is aligned by the same affine transform as the matching minutiae.

Overlapping area in pixels: 55815

OF measurement points (every 8th pixel vertically and horizontally which belongs the overlapping area): 876

Mean OF deviation: $43.1^{\circ}$

Number of deviations $>45^{\circ}: 431$

Rotation for alignment: $102.7^{\circ}$

Matched minutiae: 5

\subsubsection{Features}

For the revaluation, we aim to make good use of the already available information. From the preprocessing stage, there are the orientation field and ridge frequency estimations. From the matching stage, VFM provides some details additional to the score: the information how many and which minutiae were matched as well as the corresponding alignment (which could easily be deduced from the minutiae pairs if it would be missing). In our tests, the OF and RF estimations belonging to enhancement version E2 are applied. Out of these pieces of information, the following features are derived:

- Size of the area in pixels which is covered by both OFs after alignment by the same affine transformation as the minutiae

- Number of measurement points (every 8th pixel vertically and horizontally 
which belongs the overlapping area)

- Number of orientation deviations $>45^{\circ}$ between both OFs at measurement points

- Number of orientation deviations $>30^{\circ}$ between both OFs at measurement points

- Number of orientation deviations $>20^{\circ}$ between both OFs at measurement points

- Mean OF deviation computed over all measurement points

- Number of matched minutiae

- Number of matched minutiae which are located close to a core point

- Number of unmatched minutiae located inside the overlapping area

- Rotation for alignment for all measurement points

- Mean inter-ridge distance difference computed over all measurement points

Inspecting the false accepts and false rejects, some general rules can be formulated using above listed features: in impostor recognition attempts, a small number of randomly matching minutiae may be found on matching two templates of different fingers (see e.g. Figure 8.1). If the OFs and RF images of corresponding fingerprints are aligned using the same rotation and translation as for the minutiae, the impostor recognition attempt is characterized by not fitting OFs and RF images, whereas in genuine recognition attempts, OFs and $\mathrm{RF}$ images fit quite well.

In order to measure the goodness of fit, aforementioned features are computed. As OF foreground, we regard all pixels which are endowed with an orientation estimation, and accordingly, the RF foreground consist of all pixels with a RF estimation. After the alignment of the second OF to the first OF, we focus on the overlapping area of both OFs and measure the OF and RF deviations, count the number of unmatched minutiae as well as the number of matched minutiae which located in a core area.

\subsubsection{Case Study: FVC 2002 Database 2}

Basis for our case study are the false accepts and false rejects of FVC 2002 database 2 which remain if score fusion is performed on the scores obtained by VFM cross matching the templates of the original images and enhancement version E1 (see Section 8.4). FVC 2002 database 2 was chosen, because the lowest EER was achieved on that database and it appeared to be the easiest manageable of the twelve databases. Of the three available matching algorithms, VFM was applied for this study, because the pairs of matched minutiae and the corresponding alignment could be accessed additional to the matching score. Subsequently, we derive criteria from the properties of the remaining verifications errors in a natural way. 

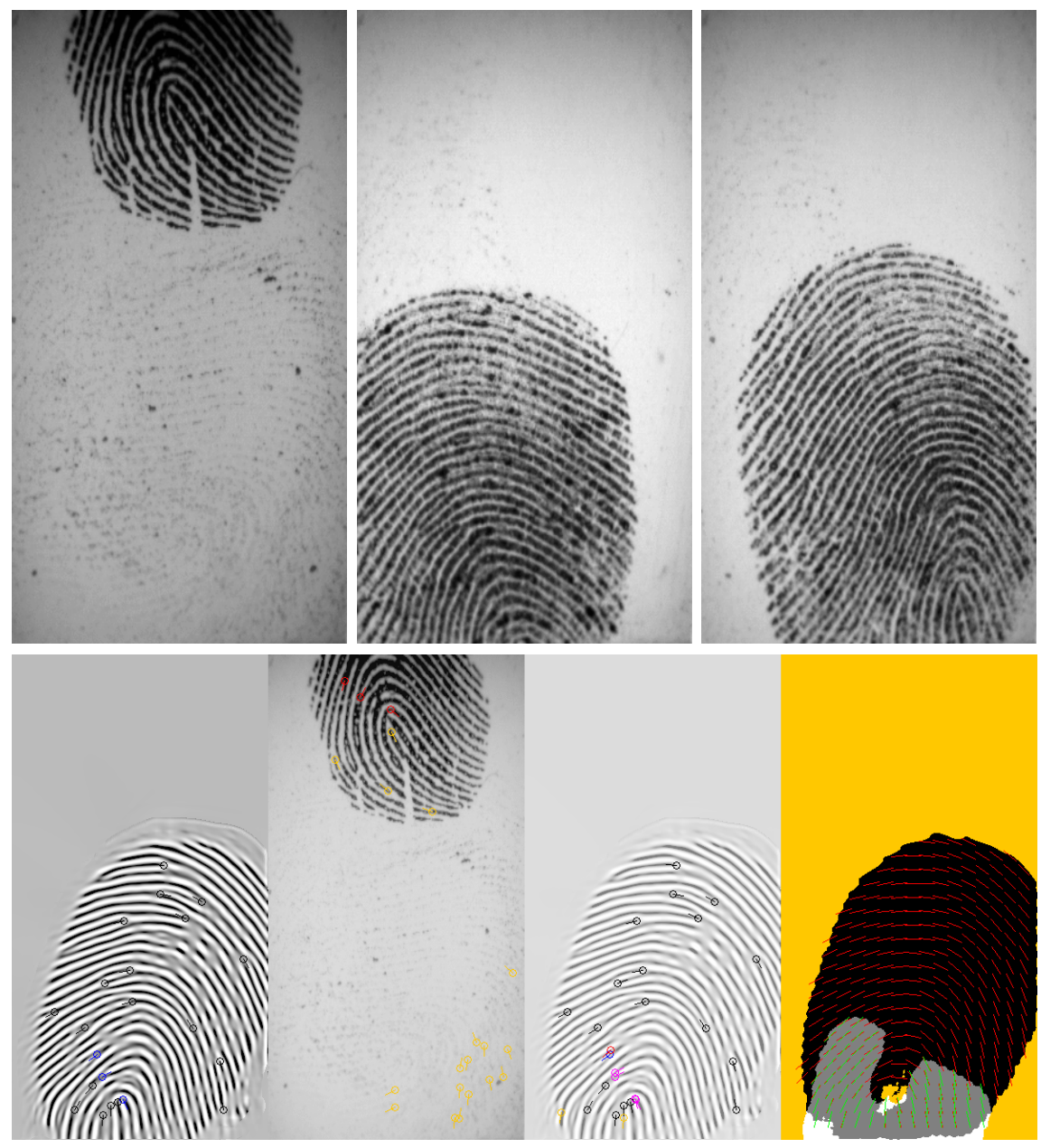

Figure 8.2: Impressions 6 to 8 (top row, from left to right) of finger 32 in FVC 2002 database 2. The genuine recognition attempt matching impression 6 and 7 achieved the lowest genuine score (17, VFM, maximum of cross matching original images and enhancement version E1) before score revaluation, the combination of impression 6 and 8 achieved the fifth lowest genuine score (25). Cause for the low scores is the small overlapping area between impression 6 and the other images after the correct alignment. Bottom row: impressions 5 and 6 are matched. Due to the small overlapping area (gray on the right, 16707 pixels) and the fitting OFs (applying rule 1 and 2), score revaluation boosts the score of this genuine recognition attempt up to 32 . 


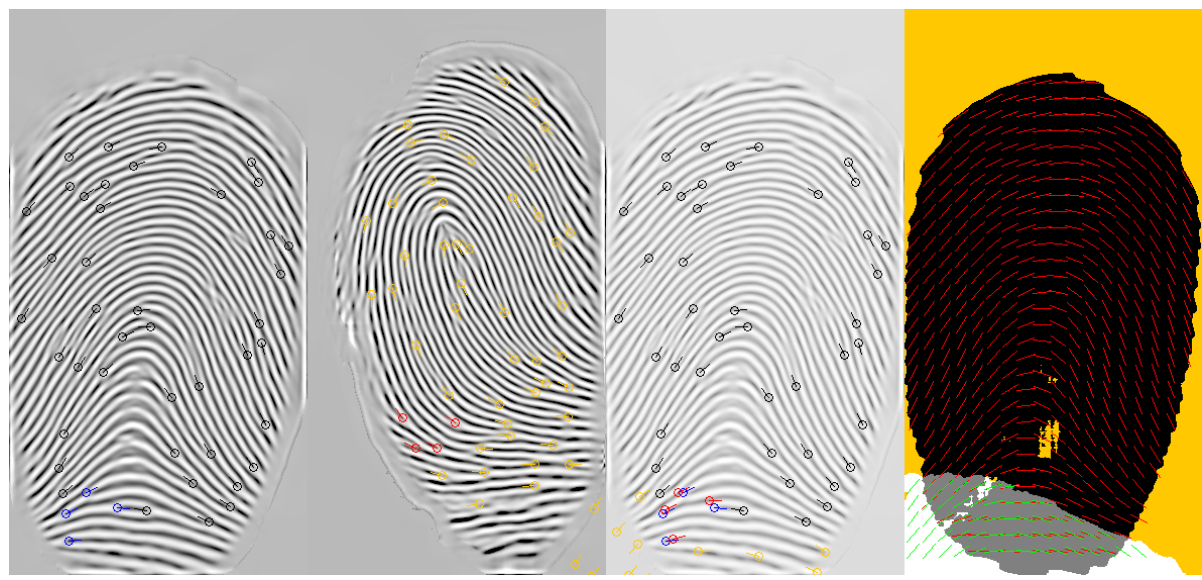

Figure 8.3: In this impostor recognition attempt of finger 26 and 100, the original score of 19 is increased, because of the small overlap (rule 1, 11996 pixels). Here, the impostor can not be recognized by non-fitting OFs (no deviations $>45^{\circ}$, mean $\mathrm{OF}$ difference is $<10^{\circ}$ ), but the rotation angle of $128^{\circ}$ is suspicious. According to rule 4 , the score is decreased by $20 \%$. Also, the inter-ridge distance difference is 2.8 , so that rule 5 applies. After score revaluation, this impostor recognition attempt is assigned a score of 24 which is the second highest of all impostor scores.

For each cross combination of original and enhanced images, a score revaluation factor is computed. At the beginning, the factor is set to $100 \%$. Depending on which conditions are met by properties of the cross combination, the factor is increased or decreased before finally the score is multiplied with the factor.

Rule 1: orientation field The orientation field is of great discriminative power. The following penalties are inflicted for deviations:

If the number of orientation deviations $>45^{\circ}$ (Dev45) between both OFs is in the range from 1 to 9 , the factor is decreased by $10 \%$, for a deviation count in the range from 10 to 49 (see e.g. Figure 8.5), the factor is halved, and for a Dev45 of 50 or more, the factor is diminished by $70 \%$.

Moreover, if the average deviation between the OFs is $\geq 10^{\circ}$, the factor is decreased by $10 \%$.

In my opinion, it is not necessary to additionally consider the number and location of core and delta points, since this information is already the contained in the orientation field. The same argument holds for the type of the pattern which is often described by the Galton-Henry-classes. Furthermore, ridge curvature correspondence which has been proposed for matching (see e.g. [92]) is contained in the $\mathrm{OF}$.

Rule 2: overlap The size of the overlapping area can have a big impact on the similarity score. A small overlap tends to cause false rejects as illustrated in 
Figure 8.2. Here, we strive to avoid these false rejects by boosting small overlaps, and in doing so, we consider the operational quality of the two fingerprints. The following thresholds are stated as absolute pixel values. Subsequently, this is generalized.

Overlapping areas (OAs) which are smaller than 5000 and simultaneously have no $\mathrm{OF}$ deviations $>45^{\circ}$, are boosted by $250 \%$. OAs between 5000 and 10000 with Dev45 = 0 are upgraded by $150 \%$, OAs between 10000 and 15000 with Dev $45=0$ are magnified by $75 \%$ and those OAs between 15000 and 40000 with Dev $45=0$ scaled up by $10 \%$.

At the other end of overlap sizes, huge overlaps which perfectly fit should be rewarded and misfits punished: OAs $\geq 80000$ with Dev $45=0$ are scaled up by $10 \%$ and OAs $\geq 80000$ with Dev45 $\geq 1$ are reduced by $25 \%$.

Rule 3: minutiae A very high number of matched minutiae (MM) should be additionally rewarded: If MM is between 15 and 19, the factor is increased by $50 \%$ and for $\mathrm{MM} \geq 20$, the upgrade is $200 \%$.

Scores should be pushed even further, if minutiae can be matched on a small overlap and the OFs perfectly fit on the overlap: If $\mathrm{MM} \geq 7$ and $\mathrm{OA}<40000$ and $\operatorname{Dev} 45=0$, then the factor is magnified by $75 \%$ and for $\mathrm{MM} \geq 7$ and $\mathrm{OA}$ $\geq 40000$ and $\operatorname{Dev} 45=0$, the increase is $20 \%$.

Inspecting the false accepts, it can be observed that a number of wrongly matched fingers contain minutiae located close to the core among the matched minutiae. Therefore, we downgrade this configuration: If OA $\geq 40000$ and there a one or more core minutiae among the matched ones, the factor is decreased by $30 \%$.

In relation to our considerations of boosting small overlaps, we also promote scenarios in which there are more matched than unmatched minutiae (UM). Typically, this is good indicator for a genuine recognition attempt with a small overlap. Here, these are boosted by $20 \%$, if $\mathrm{MM} \geq \mathrm{UM}$ and $\mathrm{MM} \geq 3$. On the other hand, a high count of UM is characteristic for an impostor recognition attempt with a huge overlap. This configuration is treated with an additional penalty: for $U M \geq 60$, the factor is reduced by $15 \%$ (see Figure 8.4 ).

Rule 4: rotation Although generally large rotations should be allowed, it can be observed that a big rotation angle is typical for many impostor recognition attempts (see e.g. Figure 8.3). Therefore, angles $\geq 60^{\circ}$ are punished by a reduction of $20 \%$.

Rule 5: ridge frequency If the inter-ridge distance deviates by more than 1 , the scaling factor is lessened by $10 \%$. 


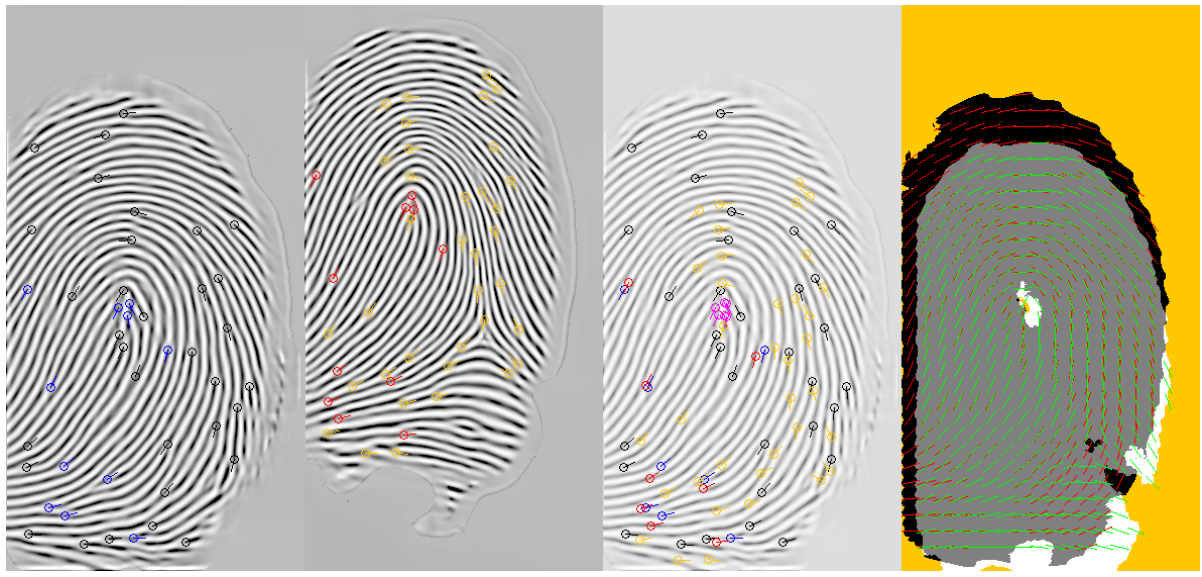

Figure 8.4: VFM finds 11 matching minutiae in this impostor recognition attempt (fingers 35 and 61 ) leading to an initial score of 34 and a false accept. At first sight comparing the two images on the left, it might not be clear whether both impressions belong to the same finger, since both prints have the same pattern (left loop), but score revaluation detects a number of misfits: there are 39 OF deviations $>45^{\circ}$ (rule 1 ), a huge overlap combined with OF deviations (rule 2), 61 unmatched minutiae (rule 3), three of the matched minutiae are located in the core area (rule 3) and the mean inter-ridge difference is 1.1 (rule 5). After score revaluation, the new score is 7 .

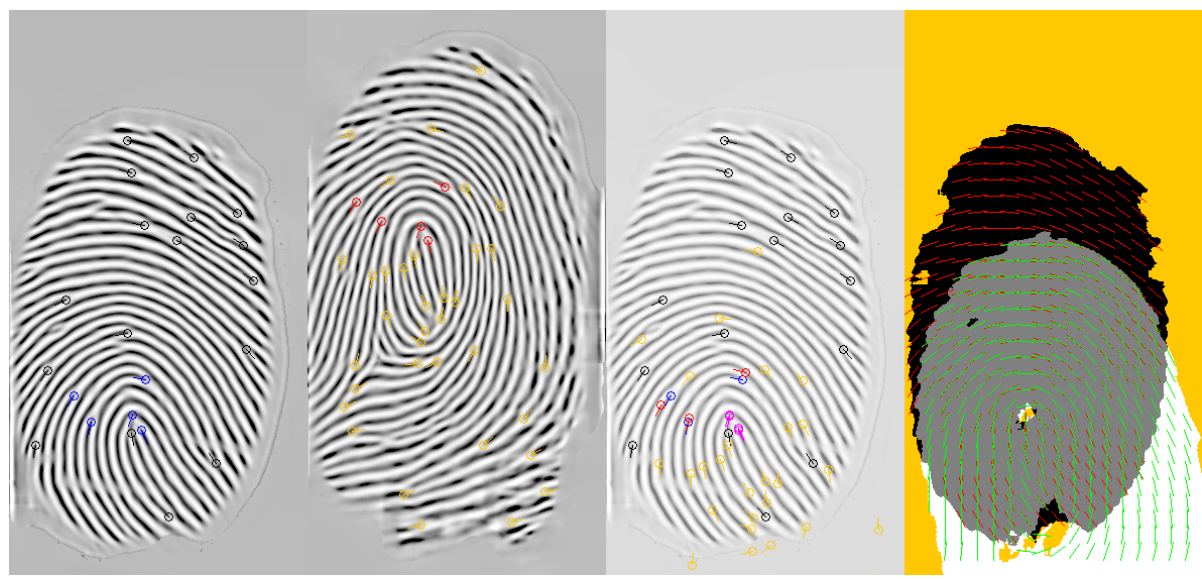

Figure 8.5: Before score revaluation, this impostor recognition attempt (fingers 16 and 77 ) with 5 matched minutiae is assigned a score of 43 by VFM. Because of $13 \mathrm{OF}$ deviations $>45^{\circ}$, the score is halved (rule 1). Two of the matched minutiae are located close to core (magenta in the third image from the left), therefore the score is reduced another $30 \%$ (rule 3). Finally, an average interridge distances of 1.3 induces an additional reduction of $10 \%$ (rule 5 ). The resulting score is 14, clearly below the threshold of 26 . 
Results and Discussion Applying aforementioned rules to the scores of the cross match combinations of the original images and enhancement version 1 leads to a perfect, error free separation into genuine users and impostors for FVC 2002 database 2. This demonstrates that the information content of at least these 800 fingerprint images of 100 fingers is sufficient for a successful separation. Nevertheless, this case study can only be regarded as a first step towards designing an AFIS that addresses the pattern recognition challenge and reliably extracts the information required for separating of genuine users and impostors on much larger databases.

Reflecting on the achieved results, it is important to be aware of the risk of overfitting. Are the applied rules generally a good choice or are they too well adapted to the specifics of FVC 2002 database 2? The question of generalizing the score revaluation criteria is covered in the next section.

\subsubsection{Generalization of Revaluation Criteria}

As a first step towards generalization, fixed thresholds from the previous section, e.g. pertaining to the overlapping area, are replaced by percentages, e.g. of the average overlapping area (AOA) of all recognition attempts for each database. E.g. AOA of FVC 2002 database 2 is 72174 pixels, AOA of FVC 2000 database 4 is 28052, and so on. Applying the aforementioned five rules to other FVC databases leads using the same thresholds leads to considerable EER reductions on all FVC databases with the exception of FVC 2004 databases 1 and 3. Subsequently, these five rules are simplified and disentangled, before different combinations of parameters and their impact are tested. Each of the following nine rules is based on one feature or the relation of two features:

Rule 1: Orientation deviations DEV45 $>0$ are devaluated. Parameters: DEV45 threshold and reduction in percent.

Rule 2: Mean orientation deviation is devaluated.

Parameters: threshold for mean orientation deviation and reduction in percent.

Rule 3: Small overlaps are boosted.

Parameters: overlap threshold in percent of AOA and increase in percent.

Rule 4: Many matched minutiae are boosted.

Parameters: matched minutiae threshold and increase in percent.

Rule 5: Scenario of more matched than unmatched minutiae is boosted. Parameters: increase in percent.

Rule 6: Core minutiae among the matched minutiae are devaluated. Parameters: core minutiae threshold and reduction in percent.

Rule 7: Many unmatched minutiae are devaluated.

Parameters: unmatched minutiae threshold and reduction in percent. 
Rule 8: Huge rotation angles are devaluated.

Parameters: angle threshold and reduction in percent.

Rule 9: Huge mean inter-ridge distance deviations are devaluated.

Parameters: mean inter-ridge distance threshold and reduction in percent.

Due to computational and time constraints, only a small number of parameter choices (3-8 per rule) has been tested so far. Results are listed in Table 8.7. It would be of interest to test additional rules, e.g. boosting combinations of huge overlap and a fitting OF. Moreover, further parameter combinations should evaluated more systematically, and new features can be added, e.g. in some areas of two fingerprints in a genuine recognition attempt agree not only in respect to the orientation and ridge frequency, but also on the absence of minutiae which can be used as a feature for boosting fitting, minutiae-free overlaps.

\subsubsection{Discussion}

In Section 8.8.2, we began our investigation with a case study of FVC 2002 database 2 and we determined criteria which optimized the performance on that database. Having generalized this approach, we are now searching for criteria that minimize the verification errors on all FVC databases.

Score revaluation accounts for the operational quality by boosting small overlaps, but image quality is not yet account for. Preferably, local and global image quality estimations will be integrated in the near future. Two additional aspects which can impact the verification and identification performance, but are not yet considered are nonlinear distortion [128, 129] and fingerprint alteration [34]. Especially nonlinear distortion may cause orientation deviations in otherwise fitting alignments of two OFs. The further development of score revaluation should make allowances for distortions in an adequate way. 


\begin{tabular}{|c|c|c|c|c|}
\hline FVC 2000 & Database 1 & Database 2 & Database 3 & Database 4 \\
\hline Original images & $4.5 \%$ & $1.05 \%$ & $8.04 \%$ & $3.77 \%$ \\
\hline Enhancement E1 & $1.65 \%$ & $0.74 \%$ & $4.61 \%$ & $2.6 \%$ \\
\hline $\begin{array}{l}\text { Cross matching of } \\
\mathrm{O} \text { and E1 }\end{array}$ & $1.08 \%$ & $0.66 \%$ & $3.69 \%$ & $1.63 \%$ \\
\hline $\begin{array}{l}\text { Score revaluation of } \\
\text { cross matching } \\
\mathrm{O} \text { and E1 }\end{array}$ & $0.48 \%$ & $0.36 \%$ & $2.58 \%$ & $0.75 \%$ \\
\hline $\begin{array}{l}\text { EER change comp. } \\
\text { original images and } \\
\text { score revaluation }\end{array}$ & $-89.3 \%$ & $-65.7 \%$ & $-67.9 \%$ & $-80.1 \%$ \\
\hline FVC 2002 & Database 1 & Database 2 & Database 3 & Database 4 \\
\hline Original images & $0.76 \%$ & $1.08 \%$ & $2.9 \%$ & $2.09 \%$ \\
\hline Enhancement E1 & $0.95 \%$ & $0.46 \%$ & $2.31 \%$ & $1.23 \%$ \\
\hline $\begin{array}{l}\text { Cross matching of } \\
\mathrm{O} \text { and E1 }\end{array}$ & $0.72 \%$ & $0.25 \%$ & $1.23 \%$ & $0.79 \%$ \\
\hline $\begin{array}{l}\text { Score revaluation of } \\
\text { cross matching } \\
\mathrm{O} \text { and E1 }\end{array}$ & $0.32 \%$ & $0.06 \%$ & $0.68 \%$ & $0.33 \%$ \\
\hline $\begin{array}{l}\text { EER change comp. } \\
\text { original images and } \\
\text { score revaluation }\end{array}$ & $-57.9 \%$ & $-94.4 \%$ & $-76.6 \%$ & $-84.2 \%$ \\
\hline FVC 2004 & Database 1 & Database 2 & Database 3 & Database 4 \\
\hline Original images & $8.29 \%$ & $7.1 \%$ & $7.35 \%$ & $2.38 \%$ \\
\hline Enhancement E1 & $6.87 \%$ & $4.18 \%$ & $4.37 \%$ & $2.29 \%$ \\
\hline $\begin{array}{l}\text { Cross matching of } \\
\mathrm{O} \text { and E1 }\end{array}$ & $5.32 \%$ & $3.84 \%$ & $3.37 \%$ & $1.33 \%$ \\
\hline $\begin{array}{l}\text { Score revaluation of } \\
\text { cross matching } \\
\mathrm{O} \text { and E1 }\end{array}$ & $4.22 \%$ & $2.82 \%$ & $2.11 \%$ & $1.01 \%$ \\
\hline $\begin{array}{l}\text { EER change comp. } \\
\text { original images and } \\
\text { score revaluation }\end{array}$ & $-49.1 \%$ & $-60.3 \%$ & $-71.3 \%$ & $-57.6 \%$ \\
\hline
\end{tabular}

Table 8.7: Overview of achieved EER reductions after enhancement, cross matching and score revaluation applying VFM. For score revaluation, the EER corresponding to the best parameter choice of the tested combinations is stated (see Section 8.8.3). 


\subsection{Conclusion and Future Work}

The second part of the thesis started in Chapter 4 with a survey of existing methods for orientation field estimation and the introduction of the line sensor method. Retrospectively, the importance of the orientation field estimation becomes even more apparent. Estimated in the primary processing step, the OF has a huge impact on all subsequent steps and the overall performance. In our sample application, the quality of the OF estimation influences the ridge frequency via the curved regions and in doing so, it influences directly and indirectly the image enhancement by the curved Gabor filter. Minutiae extraction and matching were performed by an external module, otherwise the OF could be used e.g. for alignment [149] during a latter step. In the post-matching stage, the $\mathrm{OF}$ is the key factor for the score revaluation as introduced in the previous section. The vast majority of impostors is characterized by a deviating OF after alignment and consequently, the score which is nothing but a similarity measure is accordingly devaluated. The line sensor method is an advancement in comparison to existing state of the art methods. If the verification performance on the FVC databases is used as an performance measure, then a major improvement pertaining to the quality of the OF estimation was achieved by combining the line sensor and the gradients based method in the frame of multi-level judgment aggregation. Here, improvement means that the number of erroneous estimates is significantly reduced. Nevertheless, visual inspection of the remaining false accepts and false rejects after score revaluation shows incorrect OF estimations in cases where the true ridge flow is clearly recognizable for a human expert. Bearing the importance of the OF estimation in mind, a better overall performance is to be expected by further improvements of the OF estimation.

The beauty of the presented architecture is that possibilities for improvement exist on all levels:

- Local quality estimation A locally adaptive estimation of quality is a very important aspect which is not considered in this thesis. Preferably, the estimation should not only quantify the local quality, but also classify the type of noise in case of low quality. The result of this step can have a huge positive impact on other steps. During the image enhancement, the local quality can steer the curved Gabor filter or alternative methods. At the minutiae extraction and matching stages, the quality estimation can help to avoid the extraction of false minutiae or to extenuate the influence of unreliable minutiae on the matching score. Correspondingly, weights of minutiae (and perhaps also OF and RF estimations) from low quality regions can be reduced at score revaluation.

- Orientation field estimation As sketched in Section 8.1, it is desirable to incorporate further OF estimation methods like e.g. based on quadratic Bezier curves, Gabor filter banks, 2D sine waves, normal vectors or global models. This could not only improve the OF estimation, but also, depending on the properties of the specific method, local quality could be inferred from responses of the methods.

- Ridge frequency estimation The ridge frequency estimation based on curved regions is a major advancement in comparison to methods like the 
x-signature. The exact amount of improvement should measured and it is great interest to systematically evaluate the performance of all existing methods which, to my knowledge, has not been done yet. Based on these results, possibilities for judgment aggregation on the ridge frequency can be considered.

- Image enhancement The performance of the curved Gabor can be further improved if its smoothing behavior is steered by local quality information and a local minutia probability, e.g. derived from the raw RF image. Additionally, the combination of different methods on the image enhancement level should be evaluated. E.g. Images enhanced by the curved Gabor filter, anisotropic diffusion and pyramid-based techniques may be cross matched.

- Minutiae extraction In this thesis, minutiae extraction was performed by an external software module acting as a black box. In order to enable communication between all levels, this black box should be replaced by a transparent implementation which can make good use of the already existing information like e.g. OF estimation, RF estimation and local quality.

- Alignment The available information is also useful for (pre-)aligning two images of a recognition attempt. Either by directly applying the OF [149], or by computing a symmetry axis [62] or reference points [75, 89]. Of course, if more information currently used for score revaluation can be directly applied at the minutiae extraction, alignment and matching stages, then this integration could replace the score revaluation.

- Minutiae matching One of the biggest limitations encountered during score revaluation is the output of the matcher. The software used in this thesis prints only the best result: a score and the corresponding list of matched minutiae. Preferably, a list of all scores and the appertaining minutiae pairs should be announced, because for genuine recognition attempts, the alignment belonging to the highest score may be recognized as wrong by the score revaluation in some cases due to e.g. small overlap or image quality and the corresponding devaluation may cause an avoidable false reject. In this scenario, one of the alternatives on the list could have the fitting alignment and consequently be boosted by score revaluation. Currently this information is lost, because the software outputs only the details for the matching variant endowed with the highest score which causes avoidable false rejects. Cross matching as described in Section 8.7 attempts to mitigate the negative effects of this limitation. Simultaneously, cross matching tries to make up for the lack of local quality estimation by integrating images which are smoothed using different parameters and it allows to incorporate completely different image enhancement methods. On the other hand, minutiae fusion could be an alternative to cross matching, if only the matching software would output secondary matches. Then, wrongly matched minutiae could be detected and sorted out by score revaluation. A possible workaround for using minutiae fusion in the current situation of a limited matcher output would be the following procedure: minutiae templates $\mathrm{A}$ and $\mathrm{B}$ are matched, the score and 
the corresponding list of matched minutiae are stored. Next, one of the matched minutiae is deleted from template $\mathrm{A}$, and the modified template is matched with template B. Again, the score and the list of matched minutiae are stored. The deletion step is iterated as long as the score is above a threshold. Afterwards, the course of action is repeated using the original template A and minutiae are deleted from template B. Finally, the list of scores, matched minutiae and the corresponding alignments is processed by score revaluation. The maximum score after revaluation is assigned to the recognition attempt. In the long run, score revaluation should integrated into the matching algorithm which will be a subject of future work.

- Global quality estimation As demonstrated in Section 8.5, the verification performance can be improved by considering the global quality of an image. In the context of matching and score revaluation, the 'global' should not refer to the complete image, but rather to whole overlapping area. The global measure may be derived from the previously computed local quality estimations for the corresponding region.

- Post-matching score revaluation Incorporating operational quality by boosting small overlaps and sorting out false accepts by measuring the degree of orientation field fitting are two key factors for the success of score revaluation. Further refinement on this level is possible by a thorough inspection of the remaining verification errors. Potentially, there are currently unused features within the gathered information. On the other hand, this analysis can also yield valuable hints for weaknesses and their emendations on other levels.

- Score, rank and decision level In this thesis, we turned our attention to minutiae-based matching. Of course, alternative approaches should be considered and integrated under the roof of multi-level judgment aggregation. E.g. on the score level, minutiae-based matching can be combined with correlation-based algorithms, ridge-based matching, Gabor-featurebased matching and other methods. All techniques which perform an alignment can benefit from a score revaluation before fusion. The orientation field, the derived overlap and the ridge frequency image can be utilized for all algorithms as well as quality estimation in future versions. Additional features depend on the specific approach.

In view of all these possibilities for improvements, it will be a delight to face two research challenges in fingerprint recognition: first, to reliably extract information from low quality images and to achieve very low error rates on databases like FVC 2004 [101] and NIST SD27 [43]; and second, to achieve perfect results on large databases of good quality images, and in doing so, to push the boundary of our practical knowledge of individuality further and further.

For the next generation of fingerprint recognition systems, security and template protection will be of increasing significance [72]. If fingerprints are to be employed as biometrics keys, the key length is related to the information content [121]. Naturally, the more pieces of discriminative information can be extracted and used, the less vulnerable the system will be to attacks [111]. In 
that sense, the architecture and methods discussed in this theses also pave the way for cryptographic applications of fingerprints.

Score revaluation achieved massive EER reductions in comparison to conventional template matching (see Section 8.7) by incorporating additional information, especially the orientation field, but also the ridge frequency image and derived features like the overlapping area of the two aligned OFs, the number of unmatched minutiae within the overlap etc. Therefore, I propose to add this information to templates of next generation fingerprint recognition systems. Keeping in mind the goal of minimizing template size, it would be preferable to compress the $\mathrm{OF}$ as much as possible. Global models may be applied for this purpose, e.g. the global model based on quadratic differentials [64] requires five geometrically interpretable parameters plus all singular points for computing the OF and the minutiae locations and their directions may used for fine-tuning. The application of characteristic ridges [95], e.g. modeled by quadratic Bezier Splines, could provide an alternative to this end, and the OF may be reconstructed iteratively from a curve stored in the template, analogously to the 'broadcasting' in Section 4.4.2, and by considering the divergence at minutiae locations. A third possibility is to store orientation and RF information for additional points, especially covering the area around singular points and the border of the region of interest, so that the OF and RF image can be reconstructed by a simple interpolation using the minutiae and the additional points, e.g. by a Delaunay triangulation. The design of templates endowed with additional information which are efficient with respect to computational efforts and storage size is a topic for future research. 



\section{Bibliography}

[1] Regulation (EC) No 390/2009 of the European Parliament and of the Council of 23 April 2009 amending the Common Consular Instructions on visas for diplomatic missions and consular posts in relation to the introduction of biometrics including provisions on the organisation of the reception and processing of visa applications. Official Journal of the European Union, L 131, vol. 52, pp. 1-10, 2009.

[2] "Strengthening Forensic Science in the United States: A Path Forward", Committee on Identifying the Needs of the Forensic Sciences Community, National Research Council, The National Academies Press, Washington, DC, USA, 2009.

[3] H. Ailisto, M. Lindholm and P. Tikkanen, "A Review of Fingerprint Image Enhancement Methods", International Journal of Image and Graphics, vol. 3, no. 3, pp. 401-424, 2003.

[4] F. Alonso-Fernandez, J. Fierrez-Aguilar, J. Ortega-Garcia, J. GonzalezRodriguez, H. Fronthaler, K. Kollreider and J. Bigun, "A Comparative Study of Fingerprint Image-Quality Estimation Methods", IEEE Transactions on Information Forensics and Security, vol. 2, no.4, pp. 734-743, 2007.

[5] F. Alonso-Fernandez, J. Fierrez-Aguilar and J. Ortega-Garcia, "An Enhanced Gabor Filter-based Segmentation Algorithm for Fingerprint Recognition Systems", Proc. 4th Int. Symposium on Image and Signal Processing and Analysis (ISPA2005), Zagreb, Croatia, pp. 239-244, 2005.

[6] V. Areekul, U. Watchareeruetai, K. Suppasriwasuseth and S. Tantaratana, "Fast Separable Gabor Filter Realization For Fast Fingerprint Enhancement", Proc. Int. Conf. on Image Processing (ICIP2005), Genova, Italy, pp. 253-256, 2005 .

[7] W. J. Babler, "Embryologic Development of Epidermal Ridges and Their Configurations", Birth Defects Original Article Series, vol. 27, no. 2, pp. 95-112, 1991.

[8] J. G. Barnes, "History", Chapter 1 in: "The Fingerprint Sourcebook", by the Scientific Working Group on Friction Ridge Analysis, Study and Technology (SWGFAST), National Institute of Justice, 2009. 
[9] A. M. Bazen and S. H. Gerez, "Segmentation of Fingerprint Images", Proc. ProRISC 2001 Workshop on Circuits, Systems and Signal Processing, Veldhoven, The Netherlands, 2001.

[10] A. M. Bazen and S. H. Gerez, "Systematic Methods for the Computation of the Directional Fields and Singular Points of Fingerprints", IEEE Transactions on Pattern Analysis and Machine Intelligence, vol. 24, no. 7, pp. 905-919, 2002

[11] A. M. Bazen,N. J. Bouman and R. N. J. Veldhuis, "A Multi-Scale Approach to Directional Field Estimation", Proc. ProRISC, 2004 Workshop on Circuits, Systems and Signal Processing, Veldhoven, The Netherlands, pp. 215-218, 2004

[12] A. M. Bazen, G. T. B. Verwaaijen, S. H. Gerez, L. P. J. Veelenturf and B. J. van der Zwaag, "A Correlation-Based Fingerprint Verification System", Proc. ProRISC 2000 Workshop on Circuits, Systems and Signal Processing, Veldhoven, The Netherlands, 2000.

[13] J. Berry and D. A. Stoney, "History and Development of Fingerprinting", in "Advances in Fingerprint Technology", H. C. Lee and R. E. Gaensslen (eds.), CRC Press, Boca Raton, USA, 2001.

[14] P. Bhowmick and B. B. Bhattacharya, "Removal of Digitization Errors in Fingerprint Ridgelines Using B-splines", Pattern Recognition, vol. 42, no. 3, pp. 465-474, 2009.

[15] J. Bigun, "Vision with Direction", Springer, Berlin, Germany, 2006.

[16] J. Breebaart, T. Akkermans and E. Kelkboom, "Intersubject Differences in False Nonmatch Rates for a Fingerprint-Based Authentication System", EURASIP Journal on Advances in Signal Processing, vol. 2009, article ID 896383, 2009.

[17] R. Cappelli, M. Ferrara, A. Franco and D. Maltoni, "Fingerprint Verification Competition 2006", Biometric Technology Today, vol. 15, no. 7-8, pp. $7-9,2007$

[18] R. Cappelli, D. Maio, D. Maltoni, J. L. Wayman and A. K. Jain, "Performance Evaluation of Fingerprint Verification Systems", IEEE Transactions on Pattern Analysis and Machine Intelligence, vol. 28, no. 1, pp. 3-18, 2006.

[19] R. Cappelli, D. Maio and D. Maltoni, "Semi-automatic Enhancement of Very Low Quality Fingerprints", Proc. 6th Int. Symposium on Image and Signal Processing and Analysis (ISPA), Salzburg, Austria, September 16-18, pp. 678-683, 2009.

[20] R. Cappelli, D. Maio and D. Maltoni, "An Improved Noise Model for the Generation of Synthetic Fingerprints", Proc. 8th Int. Conf. on Control, Automation, Robotics and Vision, Kunming, China, December 6-9, pp. 12501255,2004

[21] F. Chen, J. Zhou and C. Yang, "Reconstructing Orientation Field From Fingerprint Minutiae to Improve Minutiae-Matching Accuracy", IEEE Transactions on Image Processing, vol. 18, no. 7, pp. 1665-1670, 2009. 
[22] Y. Chen, S. Dass and A. K. Jain, "Fingerprint Quality Indices for Predicting Authentication Performance", Proc. AVBPA 2005, Rye Brook, New York, pp. 160-170, 2005.

[23] Y. Chen and A. K. Jain, "Beyond Minutiae: A Fingerprint Individuality Model with Pattern, Ridge and Pore Features", Proc. Int. Conf. on Biometrics (ICB), Alghero, Italy, June, 2009.

[24] Y. Chen and A. K. Jain, "Dots and Incipients: Extended Features for Partial Fingerprint Matching", Proc. Biometric Symposium, Biometric Consortium Conference, Baltimore, USA, September, 2007.

[25] S. Chikkerur, A. Cartwright and V. Govindaraju, "Fingerprint Image Enhancement Using STFT Analysis", Pattern Recognition, vol. 40, no. 1, pp. 198-211, 2007.

[26] M. M. S. Chong, R. K. L. Gay, H. N. Tan and J. Liu, "Automatic Representation of Fingerprints for Data Compression by B-spline Functions", Pattern Recognition, vol. 25, no. 10, pp. 1199-1210, 1992.

[27] L. Coetzee and E. C. Botha, "Fingerprint Recognition in Low Quality Images", Pattern Recognition, vol. 26, no. 10, pp. 1441-1460, 1993.

[28] M. J. Donahue and S. I. Rokhlin, "On the Use of Level Curves in Image Analysis", Computer Vision, Graphics, and Image Processing: Image Understanding, vol. 57, no. 2, pp. 185-203, 1993.

[29] J. G. Daugman, "Uncertainty Relation for Resolution in Space, Spatial Frequency, and Orientation Optimized by Two-dimensional Visual Cortical Filters", Journal of the Optical Society America A, vol. 2, pp. 1160-1169, 1985 .

[30] J. G. Daugman, "Biometric Decision Landscapes", Technical Report UCAM-CL-TR-482, University of Cambridge, UK, 2000.

[31] B. N. Delaunay, "Sur la Sphère Vide", Bulletin of Academy of Sciences of the USSR, vol. 7, no. 6, pp. 793-800, 1934.

[32] G. Doddington, W. Liggett, A. Martin, M. Przybocki and D. Reynolds, "Sheep, Goats, Lambs and Wolves: A Statistical Analysis of Speaker Performance in the NIST 1998 Speaker Recognition Evaluation", Proc. of Int. Conf. on Spoken Language Processing (ICSLP), vol. 4, pp. 1351-1354, 1998.

[33] T. Fawcett, "ROC Graphs: Notes and Practical Considerations for Researchers", Tech. Report, HPL-2003-4, HP Laboratories, 2003.

[34] J. Feng, A. K. Jain and A. Ross, "Fingerprint Alteration", MSU Technical Report MSU-CSE-09-30, Michigan State University, Lansing, USA, 2009.

[35] J. Fierrez-Aguilar, L. Nanni, J. Ortega-Garcia, R. Cappelli and D. Maltoni, "Combining Multiple Matchers for Fingerprint Verification: A Case Study in FVC 2004", Proc. Int. Conf. on Image Analysis and Processing (ICIAP2005), Cagliari, Italy, September 6-8, 2005. 
[36] J. Fierrez-Aguilar, J. Ortega-Garcia and Joaquin Gonzalez-Rodriguez, "Target Dependent Score Normalization Techniques and Their Application to Signature Verification", IEEE Transactions on Systems, Man and Cybernetics - Part C: Applications and Reviews, vol 35. no. 3, pp. 418-425, 2005.

[37] H. Fronthaler, K. Kollreider, J. Bigun, J. Fierrez, F. Alonso-Fernandez, J. Ortega-Garcia and J. Gonzalez-Rodriguez, "Fingerprint Image-Quality Estimation and its Application to Multialgorithm Verification", IEEE Transactions on Information Forensics and Security, vol. 3, no. 2, pp. 331-338, 2008.

[38] H. Fronthaler, K. Kollreider and J. Bigun, "Local Features for Enhancement and Minutiae Extraction in Fingerprints", IEEE Transactions on Image Processing, vol. 17, no. 3, pp. 354-363, 2008.

[39] H. Fronthaler, K. Kollreider and J. Bigun, "Pyramid-based Image Enhancement of Fingerprints", Proc. 5th IEEE Workshop on Automatic Identification Advanced Technologies (AutoID), pp. 45-50, 2007.

[40] J. Funada et al., "Feature Extraction Method for Palmprint Considering Elimination of Creases", Proc. 14th Int. Conf. Pattern Recognition, pp. 18491854,1998

[41] D. Gabor, "Theory of Communication", Journal of Institute for Electrical Engineering, vol. 93, part III, no. 26, pp. 429-457, 1946.

[42] F. Galton, "Finger Prints", MacMillan, London, 1892.

[43] M. D. Garris and R. M. McCabe, "NIST Special Database 27: Fingerprint Minutiae from Latent and Matching Tenprint Images", NIST Technical Report NISTIR 6534, 2000.

[44] S. L. Gonzaga de Oliveira, F. Viola and A. Conci, "An Approach for Enhancing Fingerprint Images Using Adaptive Gabor Filter Parameters", Pattern Recognition and Image Analysis, vol. 18, no. 3, pp. 497-506, 2008.

[45] R. C. Gonzalez and R. E. Woods, "Digital Image Processing", Prentice Hall, Upper Saddle River, New Jersey, 2002.

[46] C. Gottschlich, P. Mihăilescu and A. Munk, "Robust Orientation Field Estimation and Extrapolation Using Semilocal Line Sensors", IEEE Transactions on Information Forensics and Security, vol. 4, no. 4, pp. 802-811, 2009 .

[47] C. Gottschlich, P. Mihăilescu and A. Munk, "Robust Orientation Field Estimation in Fingerprint Images with Broken Ridge Lines", Proc. 6th Int. Symposium on Image and Signal Processing and Analysis (ISPA), Salzburg, Austria, September 16-18, pp. 529-533, 2009.

[48] C. Gottschlich, T. Hotz, R. Lorenz, S. Bernhardt, W. Krodel, M. Hantschel and A. Munk, "Modeling the Growth of Fingerprints Improves Matching for Adolescents", Unpublished Technical Report, 2010. 
[49] S. Greenberg, M. Aladjem, D. Kogan and I. Dimitrov, "Fingerprint Image Enhancement Using Filtering Techniques", Proc. Int. Conf. on Pattern Recognition (ICPR2000), September 3-8, 2000, Barcelona, Spain, vol. 3, pp. 3326-3329, 2000

[50] S. Greenberg, M. Aladjem and D. Kogan, "Fingerprint Image Enhancement Using Filtering Techniques", Real-Time Imaging, vol. 8, no. 3, pp. 227-236, 2002 .

[51] J. Gu, J. Zhou and D. Zhang, "A Combination Model for Orientation Field of Fingerprints", Pattern Recognition, vol. 37, no. 3, pp. 543-553, 2004.

[52] J. Gu, J. Zhou and C. Yang, "Fingerprint Recognition by Combining Global Structure and Local Cues", IEEE Transactions on Image Processing, vol. 15, no. 7, pp. 1952-1964, 2006.

[53] R. C. Hauspie, N. Cameron and L. Molinari (Eds.), "Methods in Human Growth Research", Cambridge University Press, Cambridge, UK, 2004.

[54] Y. He, J. Tian, X. Luo and T. Zhang, "Image Enhancement and Minutiae Matching in Fingerprint Verification", Pattern Recognition Letters, vol. 24, pp. 1349-1360, 2003.

[55] D. Henrich, "Space-efficient Region Filling in Raster Graphics", The Visual Computer: An International Journal of Computer Graphics, vol. 10, pp. 205$215,1994$.

[56] E. Henry, "Classification and Uses of Finger Prints", Routledge, London, UK, 1900.

[57] W. J. Herschel, "The Origin of Finger-Printing", Humphrey Milford, London, UK, 1916.

[58] A. Hicklin, C. Watson, and B. Ulery, "The Myth of Goats: How Many People have Fingerprints that are Hard to Match?", Technical Report NIST IR 7271, National Institute of Standards and Technology, USA, 2005.

[59] L. Hong and A. K. Jain, "Classification of Fingerprint Images", Proc. of 11th Scandinavian Conf. on Image Analysis, June 7-11, Kangerlussuaq, Greenland, 1999.

[60] L. Hong, Y. Wan and A. K. Jain, "Fingerprint Image Enhancement: Algorithms and Performance Evaluation", IEEE Transactions on Pattern Analysis and Machine Intelligence, vol. 20, no. 8, pp. 777-789, 1998.

[61] T. Hotz, "Modelling and Analysing Orientation Fields of Fingerprints", PhD Thesis, University of Göttingen, Germany, 2007.

[62] T. Hotz, "Intrinsic Coordinates for Fingerprints Based on their Longitudinal Axis", Proc. 6th Int. Symposium on Image and Signal Processing and Analysis (ISPA), Salzburg, Austria, September 16-18, pp. 500-504, 2009.

[63] C. T. Hsieh, E. Lai and Y. C. Wang, "An Effective Algorithm for Fingerprint Image Enhancement Based on Wavelet Transform", Pattern Recognition, vol 36., no. 2, pp. 303-312, 2003. 
[64] S. Huckemann, T. Hotz and A. Munk, "Global Models for the Orientation Field of Fingerprints: An Approach Based on Quadratic Differentials", IEEE Transactions on Pattern Analysis and Machine Intelligence, vol. 30, no. 9, pp. 1507-1519, 2008.

[65] B. Jähne, "Digital Image Processing", Springer, New York, 2002.

[66] A. K. Jain, "Biometric Recognition: Q\&A", Nature, vol. 449, pp. 38-40, 6 Sep. 2007.

[67] A. K. Jain, Y. Chen and M. Demirkus, "Pores and Ridges: High-Resolution Fingerprint Matching Using Level 3 Features", IEEE Transactions on Pattern Analysis and Machine Intelligence, vol. 29, no. 1, pp. 15-27, 2007.

[68] A. K. Jain and F. Farrokhnia, "Unsupervised Texture Segmentation Using Gabor Filters", Pattern Recognition, vol. 24, no. 12, pp. 1167-1186, 1991.

[69] A. K. Jain and J. Feng, "Latent Palmprint Matching", IEEE Transactions on Pattern Analysis and Machine Intelligence, vol. 31, no. 6, pp. 1032-1047, 2009 .

[70] A. K. Jain and J. Feng, "Latent Fingerprint Matching", MSU Technical Report, MSU-CSE-09-10, 2009.

[71] A. K. Jain, J. Feng and K. Nandakumar, "Fingerprint Matching", IEEE Computer, pp. 36-44, 2010.

[72] A. K. Jain and A. Kumar, "Biometrics of Next Generation: An Overview", Chapter in: Second Generation Biometrics, E. Mordini and D. Tzovaras, Eds., Springer, 2010.

[73] A. K. Jain, L. Hong and R. Bolle, "On-Line Fingerprint Verification", IEEE Transactions on Pattern Analysis and Machine Intelligence, vol. 19, no. 4, pp. 302-314, 1997.

[74] A. K. Jain, K. Nandakumar and A. Ross, "Score Normalization in Multimodal Biometric Systems", Pattern Recognition, vol. 38, no. 12, pp. 22702285,2005 .

[75] A. K. Jain, S. Prabhakar, L. Hong and S. Pankanti, "Filterbank-based Fingerprint Matching", IEEE Transactions on Image Processing, vol. 9, no. 5, pp. 846-859, 2000.

[76] A. K. Jain, N. K. Ratha and S. Lakshmanan, "Object Detection Using Gabor Filters", Pattern Recognition, vol. 30, no. 2, pp. 295-309, 1997.

[77] A. K. Jain and A. Ross, "Fingerprint Mosaicking", Proc. of IEEE Int. Conf. on Acoustics, Speech, and Signal Processing (ICASSP2002), Orlando, Florida, USA, 2002.

[78] X. Jiang, "Fingerprint Image Ridge Frequency Estimation by Higher Order Spectrum", Proc. Int. Conf. on Image Processing (ICIP00), Canada, 2000.

[79] X. Jiang, W.-Y. Yau and W. Ser, "Detecting the Fingerprint Minutiae by Adaptive Tracing the Gray-Level Ridge", Pattern Recognition, vol. 34, no. 5, pp. 999-1013, 2001. 
[80] C. Jin and H. Kim, "High-resolution Orientation Field Estimation Based on Multi-scale Gaussian Filter", IEICE Electronics Express, vol. 6, no. 24, pp. 1781-1787, 2009.

[81] S. Jirachaweng and V. Areekul, "Fingerprint Enhancement Based on Discrete Cosine Transform", Proc. of Int. Conf. on Biometrics (ICB2007), LNCS 4642, Springer, Berlin, Germany, pp. 96-105, 2007.

[82] M. Kass and A. Witkin, "Analyzing Oriented Patterns", Computer Vision, Graphics, and Image Processing, vol. 37, no. 3, pp. 362-385, 1987.

[83] M. Kawagoe and A. Tojo, "Fingerprint Pattern Classification", Pattern Recognition, vol. 17, no. 3, pp. 295-303, 1984.

[84] J. Kittler, M. Hatef, R. P. W. Duin and J. Matas, "On Combining Classifiers", IEEE Transactions on Pattern Analysis and Machine Intelligence, vol. 20, no. 3, pp. 226-239, 1998.

[85] S. A. Klein and B. Beutter, "Minimizing and Maximizing the Joint Spacespatial Frequency Uncertainty of Gabor-like Functions: Comment", Journal of the Optical Society of America A, vol. 9, no. 2, pp. 337-340, 1992.

[86] Z. M. Kovacs-Vajna, R. Rovatti and M. Frazzoni, "Fingerprint Ridge Distance Computation Methodologies", Pattern Recognition, vol. 33, no. 1, pp. 69-80, 2000.

[87] D. E. Knuth, "The Art of Computer Programming", vol. 1, pp. 308-423, Addison-Wesley, Reading, Massachusetts, 1997.

[88] R. J. Kuczmarski, C. L. Ogden, S. S. Guo et al., "2000 CDC growth charts for the United States: Methods and development", National Center for Health Statistics, Vital and Health Statistics, series 11, no. 246, 2002.

[89] H. K. Lam, Z. Hou, W. Y. Yau, T. P. Chen, J. Li and K. Y. Sim, "Reference Point Detection for Arch Type Fingerprints", Proc. of Int. Conf. on Biometrics (ICB2009), LNCS 5558, pp. 666-674, 2009.

[90] C. J. Lee and S. D. Wang, "A Gabor Filter-based Approach to Fingerprint Recognition", Proc. IEEE Workshop on Signal Processing Systems (SiPS 99), pp. 371-378, 1999.

[91] S. Lee, H. Choi, K. Choi and J. Kim, "Fingerprint-Quality Index Using Gradient Components", IEEE Transactions on Information Forensics and Security, vol. 3, no. 4, pp. 792-800, 2008.

[92] P. Li, X. Yang, Q. Su, Y. Zhang and J. Tian, "A Novel Fingerprint Matching Algorithm Using Ridge Curvature Feature", Proc. of 2009, Springer LNCS 5558, pp. 607-616, 2009.

[93] X. Liang, A. Bishnu and T. Asano, "A Robust Fingerprint Indexing Scheme Using Minutia Neighbourhood Structure and Low-Order Delaunay Triangles", IEEE Transactions on Information Forensics and Security, vol. 2, no. 4, pp. 721-733, 2007. 
[94] J. Liu, Z. Huang and K. L. Chan, "Direct Minutiae Extraction from Graylevel Fingerprint Image by Relationship Examination", Proc. Int. Conf. on Image Process., vol. 2, pp. 427-430, 2000.

[95] G. T. Mairs, "Finger Prints Indexed Numerically: a Finger Print Family Tree", Part I and II, Finger Print Identification Magazine, vol. 15, no. 4, pp. 16-18, 1933.

[96] H. Maitre, "Image Processing", ISTE Ltd, London, 2008.

[97] D. Maio and D. Maltoni, "Ridge-line Density Estimation in Digital Images", Proc. 14th Int. Conf. on Pattern Recognition (ICPR1998), Australia, pp. 1654-1658, 1998.

[98] D. Maio and D. Maltoni, "Direct Gray-Scale Minutiae Detection in Fingerprints", IEEE Transactions on Pattern Analysis and Machine Intelligence, vol. 19, no. 1, pp. 27-40, 1997.

[99] D. Maio, D. Maltoni, R. Capelli, J. L. Wayman and A. K. Jain, "FVC2000: Fingerprint Verification Competition", IEEE Transactions on Pattern Analysis and Machine Intelligence, vol. 24, no. 3, pp. 402-412, 2002.

[100] D. Maio, D. Maltoni, R. Capelli, J. L. Wayman and A. K. Jain, "FVC2002: Second Fingerprint Verification Competition", Proc. 16th Int. Conf. on Pattern Recognition (ICPR), vol. 3, pp. 811-814, 2002.

[101] D. Maio, D. Maltoni, R. Capelli, J. L. Wayman and A. K. Jain, "FVC2004: Third Fingerprint Verification Competition", Proc. Int. Conf. on Biometric Authentication (ICBA), pp. 1-7, Hong Kong, 2004.

[102] R. M. Malina, C. Bouchard, G. Beunen, "Human Growth: Selected Aspects of Current Research on Well-nourished Children", Annual Review of Anthropoly, vol. 17, pp. 187-219, 1988.

[103] D. Maltoni, D. Maio, A. K. Jain and S. Prabhakar, "Handbook of Fingerprint Recognition", Second Edition, Springer, London, 2009.

[104] D. Maltoni and R. Cappelli, "Advances in Fingerprint Modeling", Image and Vision Computing, vol. 27, no. 3, pp. 258-268, 2009.

[105] D. Marr and E. Hildreth, "Theory of Edge Detection", Proceedings of the Royal Society of London, Series B, Biological Sciences, vol. 207, no. 1167, pp. 187-217, 1980.

[106] B. M. Mehtre, "Fingerprint Image Analysis for Automatic Identification", Machine Vision and Applications vol. 6, no. 3, pp. 124-139, 1993.

[107] Y. Mei, H. Sun and D. Xia, "A Gradient-based Combined Method for the Computation of Fingerprints' Orientation Field", Image and Vision Computing, vol. 27, pp. 1169-1177, 2009.

[108] K. Mieloch, "Hierarchically Linked Extended Features for Fingerprint Processing", PhD Thesis, University of Göttingen, Germany, 2008. 
[109] K. Mieloch, A. Munk and P. Mihailescu, "Hierarchically Linked Extended Features in Fingerprints", Proc. of the 6th Biometrics Symposium (BSYM2008), pp. 47-52, 2008.

[110] P. Mihăilescu, K. Mieloch and A. Munk, "Fingerprint Classification Using Entropy Sensitive Tracing", Progress in Industrial Mathematics at ECMI 2006, pp. 928-934, 2006.

[111] P. Mihăilescu, A. Munk and B. Tams, "The Fuzzy Vault for Fingerprints is Vulnerable to Brute Force Attack", Proc. of the CAST Workshop BIOSIG, Darmstadt, Germany, Sep 17-18, 2009.

[112] A. A. Moenssens, "Fingerprint Techniques", Chilton Book Company, London, UK, 1971.

[113] M. M. Monwar and M. L. Gavrilova, "Multimodal Biometric System Using Rank-Level Fusion Approach", IEEE Transactions on Systems, Man and Cybernetics, Part B: Cybernetics, vol. 39, no. 4, pp. 867-878, 2009.

[114] K. Nandakumar, "Multibiometric Systems: Fusion Strategies and Template Security", Ph.D. thesis, Michigan State University, Lansing, USA, 2008.

[115] K. Nandakumar, Y. Chen, S. C. Dass, and A. K. Jain, "Likelihood Ratio Based Biometric Score Fusion", IEEE Transactions on Pattern Analysis and Machine Intelligence, vol. 30, no. 2, pp. 342-347, 2008.

[116] K. Nandakumar, Y. Chen, S. C. Dass, and A. K. Jain, "Quality-based Score Level Fusion in Multibiometric Systems", Proc. Int. Conf. on Pattern Recognition (ICPR2006), vol. 4, pp. 473-476, Hong Kong, 2006.

[117] S. O. Novikov and V. S. Kot, "Singular Feature Detection and Classification of Fingerprints Using Hough Transform", Proc. of SPIE, vol. 3346, pp. $270-274,1998$

[118] L. O'Gorman and J. V. Nickerson, "An Approach to Fingerprint Filter Design", Pattern Recognition, vol. 22, no. 1, pp. 29-38, 1989

[119] M. Oliveira and N. Leite, "A Multiscale Directional Operator and Morphological Tools for Reconnecting Broken Ridges in Fingerprint Images", Pattern Recognition, vol. 41, no. 1, pp. 367-377, 2008.

[120] R. Penning, "Alterskorrelierte Schätzung der Körpergröße anhand der Längen der Extremitätenknochen", Habil. thesis, Ludwig-MaximiliansUniv., Munich, Germany, 1990.

[121] R. Plaga, "Biometrics and Cryptography", Conference on Biometric Feature Identification and Analysis, Goettingen, Germany, 2007.

[122] N. Poh and S. Bengio, "F-ratio Client-Dependent Normalisation on Biometric Authentication Tasks", Proc. of Int. Conf. on Acoustics, Speech, and Signal Processing (ICASSP), Philadelphia, pp. 721-724, 2005.

[123] N. Poh, S. Bengio and A. Ross, "Revisiting Doddington's Zoo: A Systematic Method to Assess User-dependent Variabilities", Proc. of Second Workshop on Multimodal User Authentication (MMUA), Toulose, France, 2006 . 
[124] N. Poh and J. Kittler, "A Methodology for Identifying Weak Users for Controlled Enrollment and Multimodal Fusion", Proc. of 6th Biometrics Symposium, pp. 17-22, 2008.

[125] J. B. Predd, D. N. Osherson, S. R. Kulkarni and H. V. Poor, "Aggregating Probabilistic Forecasts from Incoherent and Abstaining Experts", Decision Analysis, vol. 5, no. 4, pp. 177-189, 2008.

[126] S. Ram, H. Bischof and J. Birchbauer, "Modelling Fingerprint Ridge Orientation Using Legendre Polynomials", Pattern Recognition, vol. 43, no. 1, pp. 342-357, 2010.

[127] A. Ross, "Information Fusion in Fingerprint Authentication", Ph.D. thesis, Michigan State University, Lansing, USA, 2003.

[128] A. Ross, S. Dass and A. K. Jain, "Estimating Fingerprint Deformation", Proc. of In. Conf. on Biometric Authentication (ICBA), Hong Kong, Springer LNCS 3072, pp. 249-255, 2004.

[129] A. Ross, S. Dass and A. K. Jain, "A Deformable Model for Fingerprint Matching", Pattern Recognition, vol. 38, no. 1, pp. 95-103, 2005.

[130] A. Ross, K. Nandakumar and A. K. Jain, "Handbook of Multibiometrics", Springer, New York, USA, 2006.

[131] A. Ross, A. Rattani and M. Tistarelli, "Exploiting the Doddington Zoo Effect in Biometric Fusion", Proc. of 3rd Int. Conf. on Biometrics: Theory, Applications and Systems (BTAS), Washington DC, USA, 2009.

[132] A. Ross, J. Shah and A. K. Jain, "From Template to Image: Reconstructing Fingerprints from Minutiae Points", IEEE Transactions on Pattern Analysis and Machine Intelligence, vol. 29, no. 4, 2007.

[133] F. Rothhammer, E. Llop and J. V. Neel, "Dermatoglyphic Characters and Physique: A Correlation Study", American Journal of Physical Anthropology, vol. 57, pp. 99-101, 1982.

[134] D. Salomon, "Curves and Surfaces for Computer Graphics", Springer, New York, USA, 2006.

[135] L. G. Shapiro and G. Stockman, "Computer Vision", Prentice-Hall, Upper Saddle River, New Jersey, 2001.

[136] L. L. Shen, A. Kot and W. M. Koo, "Quality Measures for Fingerprint Images", Proc. 3rd Audio and Video Based Person Authentication (AVBPA2001), pp. 266-271, 2001.

[137] B. G. Sherlock and D. M. Monro, "A Model for Interpreting Fingerprint Topology", Pattern Recognition, vol. 26, no. 7, pp. 1047-1055, 1993.

[138] P. Soille, "Morphological Image Analysis", Springer, Berlin, 2004.

[139] E. Tabassi, C. Wilson and C. Watson, "Fingerprint Image Quality", NIST Technical Report 7151, 2004. 
[140] R. Thai, "Fingerprint Image Enhancement and Minutiae Extraction", PhD Thesis, The University of Western Australia, 2003.

[141] K. Veeramachaneni, L. Osadciw, A. Ross and N. Srinivas, "Decision-level Fusion Strategies for Correlated Biometric Classifiers", Proc. of IEEE Workshop on Biometrics at the Computer Vision and Pattern Recogniton (CVPR) conference, Anchorage, USA, 2008.

[142] P. R. Vizcaya and L. A. Gerhardt, "A Nonlinear Orientation Model for Global Description of Fingerprints", Pattern Recognition, vol. 29, no. 7, pp 1221-1231, 1996.

[143] Y. Wang, J. Hu and D. Phillips, "A Fingerprint Orientation Model Based on 2D Fourier Expansion (FOMFE) and Its Application to Singular-Point Detection and Fingerprint Indexing", IEEE Transactions on Pattern Analysis and Machine Intelligence, vol. 29, no. 4, pp. 573-585, 2007.

[144] C. I. Watson, M. D. Garris, E. Tabassi, C. L. Wilson, R. M. McCabe, S. Janet and K. Ko, "User's Guide to NIST Biometric Image Software (NBIS)", National Institute of Standards and Technology, Gaithersburg, USA, 2004.

[145] Y. Wang, J. Hu and F. Han, "Enhanced Gradient-based Algorithm for the Estimation of Fingerprint Orientation Fields", Applied Mathematics and Computation, vol. 185, no. 2, pp. 823-833, 2007.

[146] J. L. Wayman, "Multi-Finger Penetration Rate and ROC Variability for Automatic Fingerprint Identification Systems", Technical Report, National Biometric Test Center, San Jose State University, USA, 1999.

[147] J. Weickert, "Coherence-enhancing Diffusion Filtering", International Journal of Computer Vision, vol. 31, pp. 111-127, 1999.

[148] N. Yager and A. Amin, "Fingerprint Verification Based on Minutiae Features: a Review", Pattern Analysis and Applications, vol. 7, no. 1, pp. 94-113, 2004.

[149] N. Yager and A. Amin, "Fingerprint Aligment Using A Two Stage Optimization", Pattern Recognition Letters, vol. 27, no. 5, pp. 317-324, 2006.

[150] N. Yager and T. Dunstone, "Worms, Chameleons, Phantoms and Doves: New Additions to the Biometric Menagerie", Proc. of Workshop on Automatic Identification Advanced Technologies AutoID, Alghero, Italy, 2007.

[151] N. Yager and T. Dunstone, "The Biometric Menagerie", IEEE Transactions on Pattern Analysis and Machine Intelligence, vol. 32, no. 2, pp. 220-230, 2010.

[152] J. Yang, L. Liu, T. Jiang and Y. Fan, "A Modified Gabor Filter Design Method For Fingerprint Image Enhancement", Pattern Recognition Letters, vol. 24, pp. 1805-1817, 2003.

[153] S. Yoon, J. Feng, and A. K. Jain, "On Latent Fingerprint Enhancement", Proc. of SPIE, Biometric Technology for Human Identification VII, 2010. 
[154] X. Zhan, Z. Sun, Y. Yin and Y. Chu, "Fingerprint Ridge Distance Estimation: Algorithms and the Performance", Proc. Int. Conf. Biometrics (ICB2006), Hong Kong, China, January 5-7, pp. 294-301, 2006.

[155] J. Zhou, F. Chen, N. Wu and C. Wu, "Crease Detection from Fingerprint Images and its Applications in Elderly People", Pattern Recognition, vol. 42, no. 5, pp. 896-906, 2009.

[156] J. Zhou and J. Gu, "Modeling Orientation Fields of Fingerprints with Rational Complex Functions", Pattern Recognition, vol. 37, no. 2, pp. 389391, 2004.

[157] J. Zhou and J. Gu, "A Model-Based Method for the Computation of Fingerprints' Orientation Field", IEEE Transactions on Image Processing, vol. 13, no. 6, pp. 821-835, 2004.

[158] E. Zhu, J. Yin, G. Zhang and C. Hu, "A Gabor Filter Based Fingerprint Enhancement Scheme Using Average Frequency", International Journal of Pattern Recognition and Artificial Intelligence, vol. 20, no. 3, pp. 417-429, 2006. 


\section{Curriculum Vitae}

Carsten Gottschlich

Born 14.10.1979 in Bückeburg

1999

Abitur at the Gymnasium Bad Nenndorf

1999-2000

Civil Service

since October 2000

Studies in Applied Computer Science at the University of Göttingen

15.08.2003

Bachelor of Science in Applied Computer Science at the University of Göttingen Gesamtnote: sehr gut

15.02.2007

Master of Science in Applied Computer Science at the University of Göttingen Gesamtnote: sehr gut

Master thesis: "Oracle 10g Rules Manager: Evaluation of the Oracle 10g Rules Manager for a Domain Node Architecture in the Semantic Web ECA Framework" supervised by Prof. Dr. Wolfgang May and Dr. Erik Behrends

since February 2007

Ph.D. Studies in Computer Science at the Institute for Mathematical Stochastics, University of Göttingen, supervised by Prof. Dr. Axel Munk.

Funded member of the DFG Research Training School 1023 "Identification in Mathematical Models: Synergy of Stochastic and Numerical Methods" 